\title{
Topologias enumeravelmente compactas em grupos abelianos
}

\author{
Irene Castro Pereira.
}

TESE APRESENTADA

$\mathrm{AO}$

INSTITUTO DE MATEMÁTICA E ESTATÍSTICA

DA

UNIVERSIDADE DE SÃO PAULO

PARA

OBTENCYAO DO GRAU

DE

DOUTOR EM MATEMÁTICA

Área de Concentração: Topologia Conjuntística

Orientador: Prof. Dr. Artur Hideyuki Tomita

Co-orientadora: Profa. Dra. Ofélia Teresa Alas

Durante a elaboração deste trabalho a autora recebeu apoio financeiro do CNPq

Sào Paulo, 18 de junho de 2004 


\section{Resumo}

Este trabalho contém exemplos e resultados sobre grupos topológicos enumeravelmente compactos.

Mostramos, a partir de um ultrafiltro seletivo sobre $\omega$, a existência de um grupo topológico enumeravelmente compacto de cardinalidade maior que $2^{\mathfrak{c}}$, sem sequências não triviais convergentes. Apresentamos uma condição suficiente para que um grupo abeliano infinito de torção admita, sob a existência de um uitrafiltro seletivo $u$, uma topologia de grupo topológico $u$-compacto sem sequências convergentes. Esta condição classifica, sob GCH, os grupos abelianos de torçào de que admitem uma topologia de grupo topológico enumeravelmente compacta e sem sequências convergentes. Mostramos também uma condição suficiente para que um grupo abeliano de torção de cardinalidade $\mathfrak{c}$, admita para cada inteiro positivo $p$, uma topologia de grupo $\tau$ tal que $(G, \tau)^{p}$ é enumeravelmente compacto e $(G, \tau)^{q}$ não é enumeravelmente compacto para algum $q>p$. Mostramos, usando forcing, que é consistente que para todo $\kappa \leq 2^{r}$, existe uma topologia de grupo enumeravelmente compacta no grupo abeliano livre de cardinalidade $\kappa$, e $r$ pode ter cofinalidade enumerável. 


\begin{abstract}
This work contains examples and results about countably compact topological groups.

We show, from a selective ultrafilter, the existence of a countably compact topological group without non-trivial convergent sequences of cardinality bigger than $2^{c}$. We present, under the existence of a selective ultrafilter $u$, a suficient condition for an infinite torsion group to admit a $u$-compact group topology without convergent sequences. This condition, under GCH, classifies the Abelian torsion groups that admit a countably compact group topology without non-trivial convergent sequences. We also show, under the existence of $\mathfrak{c}$ selective ultrafilters, a suficient condition for an Abelian torsion group of size $c$ to admit, for every positive interger $p$, a group topology $\tau$ such that $(G, \tau)^{p}$ is countably compact and $(G, \tau)^{q}$ is not countably compact for some $q>p$. We show, consistently, that for every $\kappa \leq 2^{c}$, exists a countably compact group topology on the free Abelian group of cardinality $\kappa$, and $\kappa$ may have countable cofinality.
\end{abstract}




\section{Agradecimentos}

Ao Artur por ter sugerido os problemas, pela orientacão e também pela amizade.

A Ofélia pela paciencia com tantos formulários que eu levei para assinar, e pelo apoio constante.

À Lúcia, Major, António, Samuel, porque ouviram tantas reclamaçòes e sempre me deram força para continuar.

Aproveito também este espaço para dizer que foi uma honra fazer parte do grupo de Topologia do IME-USP. Um grupo de pessoas no qual cada um torce sinceramente pelo sucesso do outro. A gente sabe que nào é fácil achar uma turma como esta.

Aos meus amigos do IME e "agregados": Cecilia, Pablo, Raul, Mário, Bárbara, Gilberto, Aldo, Liane, Daniel, Maité, Nandinho, Claus, Fábio, Marcela, Sônia. Washington, Alice, Nazaré, Sandra gaúcha, Sandra colombiana, Rudimar, Olga, Ronaldo, Gláucio, Hernán, Diane, Juan Carlos, Miguel, Claudia, e todos os que nào lembro neste momento.

A mis sobrinos, porque los dejé sin abuela por tantos meses.

Ao Felipe, mais do que um agradecimento é um pedido de desculpas, por te-lho deixado sem mãe neste final de doutorado. Espero poder recuperar o tempo perdido. Ao Zé por ser o meu companheiro em todas as horas, especialmente nas mais difíceis. A mi mamá, porque sin su ayuda incondicional nada de esto hubiera sido posible. 


\section{Sumário}

Introdução

1 Conceitos preliminares $\quad 7$

1.1 Grupos abelianos infinitos ................ 7

1.2 Teoria dos Conjuntos . . . . . . . . . . . . . . . 16

1.3 Forcing . . . . . . . . . . . . . . . . . 20

1.4 Topologia Geral . . . . . . . . . . . . . . . . . . . 23

$1.5 \beta \omega, \mathrm{P}$-pontos e ultrafiltros seletivos ........... 27

1.6 Mais sobre os espaços enumeravelmente compactos . . . . . . . . . . 29

1.7 Grupos topológicos . . . . . . . . . . . . . . . . . 31

2 Ultrafiltros seletivos e Grupos de torção $u$ - compactos 34

2.1 Sequências e ultraprodutos . . . . . . . . . . . . . . 35

2.2 Uma topologia $u$-compacta para $\mathbb{Z}(r)^{(\mathfrak{c})} \ldots \ldots \ldots . \ldots . \ldots$

2.3 Grupos de torção $u$-compactos . . . . . . . . . . . . . . . . . . 44

3 Ultrafiltros seletivos e grupos de torção enumeravelmente compactos.

3.1 Uma topologia enumeravelmente compacta para $\mathbb{Z}(r)^{(c)} \ldots \ldots 6$ 
3.2 Compacidade enumerável e grupos de torção . . . . . . . . . . . . 66

4 Um grupo abeliano livre enumeravelmente compacto cujo tamanho tem cofinalidade enumerável

A Ultrafiltros seletivos de $\omega$

B Grupos abelianos de ordem limitada e subgrupos puros

Referências Bibliográficas

99 


\section{INTRODUÇÃO}

Em 1944, Halmos mostrou que o grupo $(\mathbb{R},+)$ admite uma topologia de grupo topológico compacto, e perguntou quais grupos abelianos admitem uma topologia como esta. Em [23] Kaplansky definiu os grupos abelianos algebricamente compactos, o que permitiu avançar bastante na teoria dos grupos topológicos compactos. Por fim em 1958 e 1959, em trabalhos independentes, Harrison [18] e Hulanicki [21] responderam definitivamente a pergunta de Halmos.

A versão pseudocompacta da pergunta de Halmos foi bastante abordada. Em [12] van Douwen obteve a primeira restrição à cardinalidade $\lambda$ de um grupo topológico pseudocompacto infinito:

$$
\lambda \geq \mathfrak{c} \text {, e se } c f(\lambda)=\omega \text { então } \lambda \text { nào é limite forte. }
$$

Em [8] é exposta uma classificação de boa parte dos grupos abelianos que admitem uma topologia de grupo pseudocompacto. Mais uma vez, como no caso dos grupos compactos a solução foi dada em linguagem de invariantes cardinais.

É natural se perguntar, e assim o fazem Dikranjan e Shakhmatov no final do trabalho [8], sobre a classificaçào dos grupos que admitem uma topologia de grupo enumeravelmente compacta. Dikranjan e Tkachenko [10] obtiveram a classificação algébrica dos grupos abelianos de cardinalidade $\mathfrak{c}$ que admitem uma topologia de grupo enumeravelmente compacta, utilizando o Axioma de Martin (MA). Recentemente Dikranjan e Shakhmatov [9], baseados nas idéias de [10] obtiveram a classificação dos grupos abelianos da cardinalidade menor ou igual $2^{r}$ que admitem uma topologia de grupo enumeravelmente compacto, num modelo de forcing. 
É importante notar que, sob o Axioma de Martin, um grupo abeliano de cardinalidade $\mathfrak{c}$ admite uma topologia de grupo enumeravelmente compacta se e somente se admite uma topologia de grupo enumeravelmente compacta sem sequências nào triviais convergentes. A existência de grupos enumeravelmente compactos sem sequências não triviais convergentes ainda não foi estabelecida em ZFC, mas existem vários exemplos consistentes. O primeiro exemplo foi obtido por Hajnal e Júhasz [17] em 1976 , sob a Hipótese do Contínuo (CH). Em 1980 van Douwen [11] obteve outro exemplo sob o Axioma de Martin. Em 2000, Koszmider, Tomita e Watson [24] deram um exemplo sob MA $\mathrm{A}_{\text {countable }}$ e em 2003 Garcia-Ferreira, Tomita e Watson [15] mostraram que existe um grupo topológico enumeravelmente compacto sem sequências convergentes, a partir da existência de um ultrafiltro seletivo. Com isto, mostrou-se que o Axioma de Martin não é necessário na construção.

Neste trabalho obtivemos a classificação algébrica dos grupos abelianos de torção de cardinalidade $\mathfrak{c}$ que admitem uma topologia de grupo topológico enumeravelmente compacta sem sequências não triviais convergentes, a partir da existência de um ultrafiltro seletivo. Como GCH implica que existem ultrafiltros seletivos, a classificação comentada acima também vale sob GCH. Também apresentamos o primeiro exemplo de um grupo topológico enumeravelmente compacto sem sequências convergentes de cardinalidade maior que $2^{\mathfrak{c}}$, que por sua vez é o primeiro exemplo obtido sem usar forcing de um grupo topológico enumeravelmente compacto sem sequências convergentes de cardinalidade maior que $\mathfrak{c}$.

Em 1990, Tkachenko [27] mostrou, assumindo $\mathrm{CH}$, que existe uma topologia de 
grupo enumeravelmente compacta no grupo abeliano livre de cardinalidade $\mathfrak{c}$. Ele construiu um subgrupo livre e denso de $\mathbb{T}^{\mathrm{c}}$ que é enumeravelmente compacto. Neste trabalho $\mathbb{T}=\mathbb{R} / \mathbb{Z}$. Dikranjan e Shakhmatov [8] provaram que nenhum grupo livre (não abeliano) admite uma topologia de grupo enumeravelmente compacta. De fato, eles mostraram que todo subgrupo enumeravelmente compacto de um grupo livre Hausdorff é trivial. Motivados por este resultado perguntaram:

Para quais cardinais $\kappa$, o grupo abeliano live de cardinalidade $\kappa$, admite uma topologia de grupo enumeravelmente compacta?

Em [24] foi mostrado que é consistente que o grupo livre de cardinalidade $2^{\mathfrak{c}}$ admite uma topologia de grupo enumeravelmente compacta e, portanto, também todo grupo livre de cardinalidade $\kappa \leq 2^{\mathfrak{c}} \operatorname{con} \kappa=\kappa^{\omega}$. E se $\kappa \neq \kappa^{\omega}$ ?

van Douwen, em [12] já tinha deixado a seguinte pergunta:

Se $G$ é um grupo infinito enumeravelmente compacto, é $|G|^{\omega}=|G|$ ? É pelo menos $\operatorname{cf}(|G|) \neq \omega ?$

O próprio van Douwen, em [12], respondeu positivamente a esta pergunta sob GCH. Mas em [31], Tomita respondeu negativamente a pergunta. Ele deu um exemplo de grupo abeliano de ordem 2, enumeravelmente compacto, de cardinalidade $\aleph_{\omega}$. Este exemplo contém seqüências convergentes.

Neste trabalho mostramos que é consistente que existe uma topologia de grupo enumeravelmente compacta, sem seqüencias convergentes, no grupo abeliano livre de cardinalidade $\lambda$, para cada $\mathfrak{c} \leq \lambda \leq 2^{\mathfrak{c}}$, e que $2^{\mathfrak{c}}$ pode ser "arbitrariamente grande". 
Em particular $\lambda$ pode ter cofinalidade enumerável. Este resultado foi obtido independentemente do trabalho de Dikranjan e Shakhmatov [9].

Admitindo a existência de um grupo booleano $G$, infinito, enumeravelmente compacto, sem sequências convergentes (construído em [11] sob MA), van Douwen obteve sob MA dois grupos enumeravelmente compactos cujo produto não é enumeravelmente compacto. Ele constrói dois subgrupos $G_{0}$ e $G_{1}$ de $G$, enumeravelmente compactos tais que $\left|G_{0} \cap G_{1}\right|=\omega$, com isto $G_{0} \times G_{1}$ não pode ser enumeravelmente compacto já que contém a diagonal, um subconjunto enumerável infinito e fechado. Utilizando outra técnica Hart e van Mill [19], obtiveram, utilizando $\mathrm{MA}_{\text {countable, um grupo enu- }}$ meravelmente compacto cujo quadrado nào é enumeravelmente compacto. Ainda é um problema aberto se existe um modelo de ZFC no qual o produto de grupos enumeravelmente compactos seja enumeravelmente compacto.

No Open Problems in Topology [3], Comfort perguntou para quais cardinais $\kappa$ existe um grupo topológico $G$ tal que $G^{\lambda}$ é enumeravelmente compacto para todo $\lambda<\kappa$, mas $G^{r}$ não é. Recentemente [29] Tomita deu uma resposta afirmativa para todo cardinal finito, sob MA countable.

Neste trabalho, admitindo a existência de c ultrafiltros seletivos, também obtivemos para cada inteiro $p$, exemplos de grupos topológicos $G$ tais que $G^{p}$ é enumeravelmente compacto, mas existe $q>p$ tal que $G^{q+1}$ nào é enumeravelmente compacto .

Depois de feita a introdução histórica, vamos descrever nosso trabalho capítulo a capitulo. 
No Capítulo 1, apresentamos conceitos e propriedades sobre Grupos abelianos, Teoria dos Conjuntos e Topologia Geral, que serào utilizados com familiaridade no restante do trabalho.

No Capítulo 2, apresentamos uma condiçào suficiente para que um grupo abeliano de torção admita uma topologia de grupo topológico $u$-compacto e sem seqüências convergentes, onde $u$ é um ultrafiltro seletivo sobre $\omega$. Notamos que se $G$ é um grupo topológico $u$-compacto, então $G^{\kappa}$ é enumeravelmente compacto para todo cardinal $\kappa$. Na primeira seçào do capítulo desenvolvemos a construção para o grupo $\mathbb{Z}(r)^{(\mathfrak{c})}$ com $r$ primo, que por ser um espaço vetorial facilita as contas e achamos que permite enxergar a técnica utilizada com mais clareza.

No Capítulo 3, mostramos uma condição suficiente para que um grupo de torçào $G$ de cardinalidade $c$ admita, para cada inteiro positivo $p$, uma topologia de grupo tal que $G^{p}$ é enumeravelmente compacto, mas existe $q>p$ tal que $G^{q+1}$ nào é enumeravelmente compacto, sob a existência de $\mathfrak{c}$ ultrafiltros seletivos.

No Capítulo 4, usando forcing, construimos um subgrupo livre de $\mathbb{T}^{\mathrm{c}}$ que é enumeravelmente compacto e sem seqüências convergentes, de cardinalidade $2^{r}$.

Este trabalho possui dois apêndices. O objetivo de ambos é o mesmo: aprofundar um pouco mais em algumas das propriedades que aparecem só citadas no primeiro capítulo, com o fim de torná-las mais "naturais" para o leitor que nào está acostumado a trabalhar com elas. No Apêndice A nos concentramos nos ultrafiltros seletivos sob 
$\omega$ e no Apêndice $B$ em algumas propriedades algébricas. 


\section{Capítulo 1}

\section{Conceitos preliminares}

Neste capítulo fazemos um resumo das definições e propriedades que serão usadas neste trabalho e também fixamos algumas notaçôes.

\subsection{Grupos abelianos infinitos}

Vamos introduzir aqui os conceitos algébricos que aparecem ao longo desta tese. Nào desenvolvemos nenhuma demonstraçầo nestá seção, mas por se tratar de um tópico que não faz estritamente parte da Topologia Conjuntística, achamos conveniente incluir um apêndice para os leitores interessados em ver alguns conceitos com mais detalhe. Para estudar as propriedades dos grupos abelianos utilizamos o livro de Fuchs [14] e o livro de Kaplansky [23].

Um grupo abeliano é um par $(G,+)$ onde $G$ é um conjunto tal que para cada par de elementos $a, b$ de $G$ está associado um elemento $a+b$ de $G$, chamado soma de $a$ e $b$; existe um elemento $0 \in G$, o zero, tal que $a+0=a, \forall a \in G$; para cada $a \in G$ existe 
um elemento $x \in G \operatorname{com} a+x=0, x=-a$ é o inverso de $a$; finalmente $(G,+)$ satisfaz as propriedades associativa e comutativa: $(a+b)+c=a+(b+c)$ e $a+b=b+a$, $\forall a, b, c \in G$.

\section{Subgrupos e geradores}

A soma $a+a+\ldots+a$ de $n$ termos iguais a $a$ é abreviada por na ou n.a. Um elemento $n a, \operatorname{com} n$ inteiro é chamado um múltiplo de $a$.

Um subgrupo de $(G,+)$ é um par $(H,+)$, onde $H$ é um subconjunto de $G$, +é a restrição da operação definida em $G$ e $(H,+)$ é um grupo. Em geral, vamos omitir a operação e falar que $H$ é subgrupo de $G$. Observamos que $G$ e $\{0\}$ são subgrupos de $G$. Um subgrupo de $G$ diferente de $G$ é um subgrupo próprio.

Se $H$ é um subgrupo de $G$, seja a relação de equivalência definida em $G$ por

$$
a \sim_{H} b \Longleftrightarrow a-b \in H .
$$

então $G / H=\{a+H: a \in G\}$ com a soma definida por $(a+H)+(b+H)=(a+b)+H$ é também um grupo abeliano.

Seja $\left\{H_{i}: i \in I\right\}$ é uma família de subgrupos de $G$, então $\bigcap_{i \in I} H_{i}$ é também um subgrupo de $G$.

Se $S$ é um subconjunto de $G$, o símbolo $\langle S\rangle$ denotará o subgrupo de $G$ gerado por $S$, isto é, a interseç̧ão de todos os subgrupos de $G$ que contém $S$. Se $S=\left\{a_{i}: i \in I\right\}$, também escrevemos $\langle S\rangle=\left\langle a_{i}\right\rangle_{i \in I}$. Este grupo $\langle S\rangle$ consiste em todas a somas da forma $n_{1} a_{1}+\ldots+n_{k} a_{k}$ (combinação linear), com $a_{i} \in S$ e $n_{i}$ inteiro, e $k$ um número natural. Se $\langle S\rangle=G, S$ é dito um sistema de geradores de $G$ e os elementos de $S$ são chamados geradores de $G$. O grupo $\langle a\rangle$, é o grupo cíclico gerado por $a$. 
A ordem $o(a)$, de um elemento a do grupo, é o menor natural $n \neq 0$ tal que $n a=0$ ou $\infty$ no caso em que na $\neq 0$ para todo número natural $n \neq 0$. Se todo elemento do grupo $G$ tem ordem finita, $G$ é chamado grupo de torção, e se todo elemento não nulo de $G$ tem ordem infinita, $G$ é um grupo livre de torção. Um grupo primário é um grupo tal que todos os seus elementos tem ordem igual a potência do número primo $r$ fixado. Também é chamado de $r$-grupo.

Para um grupo $G$ e um inteiro $n$, seja

$$
n G=\{n a: a \in G\}
$$

e para $n>0$,

$$
G[n]=\{a: a \in G, n a=0\} .
$$

Então $a \in n G$ se e somente se a equação $n x=a$ tem soluçâo em $G$, e $a \in G[n]$ se e somente se $o(a) \mid n$ (a ordem do elemento $a$ divide $n$ ). $n G$ e $G[n]$ são subgrupos de $G$.

\section{Homomorfismos.}

Sejam $G$ e $H$ grupos abelianos. Um funçào $f: G \rightarrow H$ é um homomorfismo de grupos se $f(a+b)=f(a)+f(b)$ para todo $a, b \in G$. Todo homomorfismo da origem a dois subgrupos: Kerf subgrupo de $G$ e Im $f$ subgrupo de $H$. O kemel de $f$, é o conjunto dos elementos $a \in G$ que verificam $f(a)=0$, e a imagem de $f$, é o conjunto dos elementos $b$ de $H$ tais que existe algum $a \in G \operatorname{com} f(a)=b$. Vamos usar a notaçào $f(G)$ para $\operatorname{Im} f$. Se $f$ é sobrejetivo, dizemos que é um epimorfismo, se $f$ é injetivo dizemos que é um monomorfismo e se $f$ é bijetivo dizemos que é um isomorfismo. Se $f: G \rightarrow H$ é um isomorfismo, os grupos $G$ e $H$ sào isomorfos e denotamos $G \cong H$. Observamos que se $f: G \rightarrow H$ é um monomorfismo, então $G$ e $\operatorname{Im} f$ são isomorfos. 


\section{Exemplos.}

(a) Grupos cíclicos: São os grupos gerados por um único elemento. Se o elemento gerador for de ordem infinita, então o grupo cíclico gerado é isomorfo ao grupo $(\mathbb{Z},+)$ dos inteiros. Se o elemento gerador for de ordem finita $n$, então o grupo cíclico gerado é isomorfo ao grupo aditivo dos inteiros $\bmod n(a=b(\bmod n)$ se $n \mid a-b)$, o grupo $(\mathbb{Z}(n),+)$. Isto é um grupo cíclico $\langle a\rangle$ de ordem $n$ consiste dos elementos $0, a, 2 a, \ldots,(n-1) a$.

(b) Os grupos de racionais: o conjunto $\mathbb{Q}$ dos números racionais com a soma, é um grupo abeliano e é um exemplo de grupo que nào é finitamente gerado, ou seja nào possui um conjunto finito de geradores. Neste grupo temos o subgrupo $\mathbb{Z}$ dos inteiros. O Grupo quociente $\mathbb{Q} / \mathbb{Z}$ é conhecido como o grupo dos racionais mod 1. Este grupo é muito importante para a teoria de grupos divisíveis.

(c) $O$ grupo $\mathbb{Z}\left(r^{\infty}\right)$ : Seja $r$ um número primo, e seja $R$ o grupo aditivo dos números racionais cujos denominadores são potências de $r$. O grupo quociente $R / \mathbb{Z}$ é denotado por $\mathbb{Z}\left(r^{\infty}\right)$.

\section{Somas diretas.}

Sejam $F$ e $H$ subgrupos de $G$ tais que:

(i) $F+H=G$, onde $F+H=\{b+c: b \in F, c \in H\}$;

(ii) $F \cap H=\{0\}$.

Entào dizemos que o grupo $G$ é a soma direta interna de $F$ e $H$. Isto significa que todo elemento de $G$ pode se escrever de forma única como soma de um elemento de $F$ e um elemento de $H$.

Seja $\left\{H_{i}: i \in I\right\}$ um conjunto de grupos. Um vetor sobre o conjunto $\prod_{i \in I}$ tem exatamente uma coordenada $b_{i}$ para cada $i \in I, \operatorname{com} b_{i} \in H_{i}$. Este vetor pode ser 
interpretado como uma função définida no conjunto $I$ tal que $f(i)=b_{i} \in H_{i}$, para cada $i \in I$. A igualdade e soma de vetores se define coordenada a coordenada. Os vetores assim definidos formam um grupo chamado produto direto dos grupos $H_{i}$.

Se considerarmos só aqueles vetores $\left(\ldots, b_{i}, \ldots\right)$ tais que $b_{i}=0$ para todos menos um número finito de $i \in I$, temos um grupo $G$ chamado de soma direta (externa) dos grupos $H_{i}, G=\oplus_{i \in I} H_{i}$. Se $\kappa$ é um cardinal e $G$ é a soma direta de $\kappa$ cópias do grupo $H$, escrevemos $G=H^{(\kappa)}$. Se $G$ é o produto direto de $\kappa$ cópias de $H$, escrevemos $G=H^{\kappa}$.

Seja $G=\bigoplus_{i \in I} H_{i}$, e seja $a \in G$ então $a=\left(\ldots, b_{i}, \ldots\right)$ e $\left\{i \in I: b_{i} \neq 0\right\}$ é finito. Este conjunto é chamado suporte de a e vai ser denotado por supp $(a)$.

\section{Independência linear.}

Un conjunto $\left\{a_{1}, \ldots, a_{k}\right\}$ de élementos de um grupo $G$ é chamado linearmente independente ou simplesmente independente se

$$
n_{1} a_{1}+\ldots+n_{k} a_{k}=0 \quad\left(n_{i} \in \mathbb{Z}\right)
$$

implica

$$
n_{1} a_{1}=\ldots=n_{k} a_{k}=0 .
$$

Isto significa que $n_{i}=0$ se ardem de $a_{i}$ é infinita e que a ordem de $a_{i}$ divide $n_{i}$ se esta for finita. Um conjunto de elementos de $G$ é dependente se não for independente. Observamos que em geral nào é verdade que um elemento numa família dependente possa se escrever como combinação linear dos outros elementos.

Uma família infinita $L=\left\{a_{i}: i \in I\right\}$ de elementos de $G$ é chamada independente, se toda sub-familia finita de $L$ for independente. 
Lema 1.1. Uma família $L=\left\{a_{i}: i \in I\right\}$ de elementos de $G$ é independente se $e$ somente se o subgrupo gerado por $L$ é a soma direta dos grupos cíclicos $\left\langle a_{i}\right\rangle, i \in I$.

$a \in G$ depende de um subconjunto $L$ de $G$, se existe uma relação de dependência

$$
0 \neq n a=n_{1} a_{1}+\ldots+n_{k} a_{k}
$$

onde $a_{i} \in L$ e $n, n_{i}$ sào inteiros.

Uma família independente $M$ de $G$ é chamada maximal se não existe uma família independente de $G$ que contenha $M$ propriamente. O rank $r(G)$ de um grupo $G$ é a cardinalidade de uma família independente maximal que contém só elementos de ordem infinita e elementos de ordem a potência de um número primo. Se considerarmos uma família maximal só com elementos de ordem infinita, entào a cardinalidade desta família é chamada rank live de torção, $r_{0}(G)$. O $p-r a n k r_{p}(G)$ de $G$ é definido analogamente, considerando só elementos cujas ordens sào potências de um múmero primo fixo $p$.

Teorema 1.2. Os cardinais $r(G), r_{0}(G) \in r_{p}(G)$ são invariantes do grupo $G$, isto 6 não dependem da família independente maximal considerada.

\section{Grupos de torção.}

Vamos ver algumas propriedades específicas dos grupos de torção.

Teorema 1.3. Todo grupo abeliano de torçâo é soma direta de grupos primários.

De fato, se $G$ é um grupo de torção e $G_{r}$ é o subgrupo formado pelos elementos de $G$ que tem ordem uma potência de $r$, então $G=\oplus_{r} G_{r}$, onde $r$ percorre o conjunto dos números primos. Por exemplo, seja o grupo cíclico $\mathbb{Z}(n)$, onde $n$ tem decomposiçào 
em fatores primos $r_{1}^{k_{1}} \ldots r_{t}^{k_{t}}$, entào $\mathbb{Z}(n)=\mathbb{Z}\left(r_{1}^{k_{1}}\right) \oplus \ldots \oplus \mathbb{Z}\left(r_{t}^{k_{t}}\right)$.

O grupo $\mathbb{Q} / \mathbb{Z}$ é um grupo de torção e a sua componente primária para o primo $r$ é o grupo $\mathbb{Z}\left(r^{\infty}\right)$. Então $\mathbb{Q} / \mathbb{Z}$ é a soma direta de todos os grupos $\mathbb{Z}\left(r^{\infty}\right)$.

Dizemos que um grupo $G$ é de ordem limitada se é um grupo de torção e existe um inteiro positivo $n$ tal que $n G=0$ ou o que é equivalente que $G[n]=G$. Se $m=\min \{n>0: n G=0\}$ dizemos que o grupo $G$ tem ordem $m$.

Teorema 1.4. Um grupo de ordem limitada é soma direta de grupos cíclicos.

Observamos que se $L$ é um subconjunto de $G, f:\langle L\rangle \rightarrow H$ é um homomorfismo de grupos e $a \in G$ é tal que $L \cup\{a\}$ é independente, então existe um homomorfismo $g:\langle L \cup\{a\}\rangle \rightarrow H$ tal que $h \uparrow_{\langle L\rangle}=f$. Mais ainda, se a ordem de $a$ for infinita $h(a)$ pode ser escolhida entre todos os elementos de $H$, e se ord $(a)=n, h(a)$ pode ser escolhida entre todos os elementos $b \in H$ tais que ord $(b) \mid$ ord $(a)$.

\section{Grupos divisíveis e subgrupos puros.}

Dizemos que um elemento $x$ do grupo $G$ é divisivel pelo inteiro $n$, se existe $y \in G$ tal que $x=n y$. Um grupo $G$ é divisivel se para cada $x \in G$ e cada inteiro $n, x$ é divisível por $n .(G=n G, \forall n \in \mathbb{Z})$.

Um subgrupo $H$ do grupo $G$ é puro se $n H=H \cap n G \forall n \in \mathbb{Z}$.

Teorema 1.5. Um subgrupo puro de ordem limitada é um somando direto do grupo. 


\section{Invariantes de Ulm-Kaplansky.}

Sejam $r$ um número primo e $G$ um $r$ grupo. Para cada ordinal $\alpha$ definimos um subgrupo $G_{a}$ de $G$, como segue:

$G_{0}=G$

$G_{1 \alpha}=r \cdot G_{\beta}$ se $\alpha=\beta+1$,

$G_{\alpha}=\bigcap_{\beta<\alpha} G_{\beta}$ se $\alpha$ é um ordinal limite.

Então existe um ordinal $\gamma$ tal que $G_{\gamma+1}=G_{\gamma}$, e dai para a frente a sequência permanece constante. Como $G_{\gamma}=r \cdot G_{\gamma}$, temos que $G_{\gamma}$ é um subgrupo divisível de $G$, é o maior subgrupo divisível de $G$. Se $G$ não tem subgrupo divisíveis, temos uma sequência de subgrupos que começa em $G$ e acaba em 0 .

Seja. $R$ o subgrupo de $G$ formado pelos elementos de ordem $r$, e seja $R_{\alpha}=R \cap G_{\alpha}$, para cada $\alpha<\gamma$. O quociente $R_{\alpha} / R_{\alpha+1}$ é um espaço vetorial sobre $\mathbb{Z}(r)$. A dimensão $f(\alpha)$ deste espaço vetorial é chamada $\alpha$-ésima invariante de Ulm-Kaplansky de $G$.

Vamos ver um exemplo. Seja $G$ um grupo cíclico de ordem $r^{k}$. Se $n \geq k$, entào $G_{n}, R_{n}$ e $R_{n} / R_{n+1}$ são todos $\{0\}$, portanto $f(n)=0$. Se $n \leq k-2$, então $G_{n}$ é cíclico de ordem $r^{k-n}, G_{n+1}$ é seu único subgrupo de ordem $r^{k-n-1}, R_{n}$ e $R_{n+1}$ são cíclicos de ordem $r, R_{n} / R_{n+1}=0$, e $f(n)=0$. Se $n=k-1$, vemos que $f(k-1)=1$.

Baseados na idéia intuitiva deste exemplo, podemos concluir que: se $G$ é soma direta de grupos cíclicos, então a sua n-ésima invariante de Ulm-Kaplansky $f(n)$ é precisamente a quantidade de termos ciclicos de ordem $r^{n+1}$.

Portanto, para aqueles grupos que são soma direta de grupos cíclicos, as invariantes de Ulm-Kaplansky, nos dão uma caracterização do grupo.

Lema 1.6. Para um grupo abeliano $G$ de ordem $n$ e cardinalidade $c$ säo equivalentes: (i) para cada divisor próprio d de n, o subgrupo $d G$ ou é finito ou tem cardinalidade $\mathfrak{c}$ 
(ii) para cada componente primária $G_{r}$ de $G$, o último invariante infinito de UlmKaplansky de $G_{r}$ éc.

Demonstração: (i) $\Rightarrow$ (ii): Se $\lambda$ é a invariante de Ulm-Kaplansky de $G_{r}$ correspondente a $\mathbb{Z}\left(r^{k}\right)$, por hipótese temos que $r^{k} G$ ou é finito ou tem cardinalidade $\mathfrak{c}$.

$$
r^{k} G=\left\{x \in G: \exists y \in G, x=r^{k} \cdot y\right\}
$$

Se $r^{k} G$ é finito, então $\forall k^{\prime} \leq k$ a invariante de Ulm-Kaplansky correspondente a $\mathbb{Z}\left(r^{k^{\prime}}\right)$ é um cardinal finito. Se $\left|r^{k} G\right|=\mathfrak{c}$ então existe $k^{\prime} \leq k$ tal que a invariante de UlmKaplansky correspondente a $\mathbb{Z}\left(r^{k^{\prime}}\right)$ é $\mathfrak{c}$.

Portanto a última invariante infinita de $G_{r}$ é c.

(ii) $\Rightarrow$ (i): Seja $d$ um divisor próprio de $n$ e seja $r_{1}^{k_{1}} \ldots r_{s}^{k_{s}}$ a decomposiçào de $d$ em fatores primos. Se para algum $1 \leq i \leq s$ a última invariante infinita de Ulm-Kaplansky de $G_{n_{i}}$ é a correpondente a $\mathbb{Z}\left(r_{i}^{k}\right) \operatorname{com} k \leq k_{i}$ então então $|d G|=\mathfrak{c}$. Caso contrário $d G$ é finito.

\section{Grupos livres.}

Um grupo abeliano livre é um grupo que é soma direta de grupos cíclicos infinitos. Isto é, é um grupo da forma

$$
F=\oplus_{i \in I}\left\langle x_{i}\right\rangle
$$

onde a ordem de cada $x_{i}$ é $\infty$.

Entào $F$ consiste de todas as combinações lineares finitas

$$
g=n_{1} x_{i_{1}}+\ldots+n_{k} x_{i_{k}}
$$

com $x_{i_{1}}, \ldots, x_{i_{k}}$ diferentes, os $n_{j}$ inteiros não nulos, e $k$ um número natural. combinaçòes lineares são o mesmo elemento de $F$ se e somente se diferem só na ordem 
dos somandos.

Se $X=\left\{x_{i}\right\}_{i \in I}$ e $F$ é o grupo formado por todas as expressões formais como acima, dizemos que $X$ é um conjunto livre de geradores para $F$.

O grupo livre $F$ está determinado pela cardinalidade $\kappa$ do conjunto de índices $I$. Se escrevemos $F_{\kappa}$ para o grupo gerado por um conjunto livre de cardinalidade $\kappa$ temos

Proposição 1.7. Os grupos livres $F_{\kappa}$ e $F_{\lambda}$ são isomorfos se e somente se $\kappa=\lambda$.

Teorema 1.8. Um conjunto $X=\left\{x_{i}\right\}_{i \in I}$ de geradores de um grupo $F$ é um conjunto live de geradores se, e somente se, toda função $f: X \rightarrow A$, de $X$ num grupo $A$, pode ser estendida a um homomorfismo $\phi: F \rightarrow A$.

Teorema 1.9. Um subgrupo de um grupo livé é também um grupo livre.

\subsection{Teoria dos Conjuntos}

A referência utilizada para os conceitos conjuntistas é o livro de liunen [25] e o livro de Jech [22]. Trabalharemos no contexto da axiomática de Zermelo Fraenkel com o Axioma da Escolha (ZFC).

Assumiremos conhecidas as operações básicas com conjuntos assim como as noções de função, relação de ordem e boa ordem.

Vamos fixar algumas notações. $A \backslash B$ será a notação usada para a diferença entre dois conjuntos $A$ e $B$. Se $f: X \rightarrow Y$ é uma função, denotaremos $\operatorname{dom}(f)$ e $i m(f)$ o dominio e a imagem de $f$ respectivamente. Se $A \subseteq X$ e $B \subseteq Y$ então

$$
f(A)=\{f(x): x \in A\} \text { e } f^{-1}(B)=\{x \in X: \exists b \in B, f(x)=b\}
$$

A restrição da função $f$ ao conjunto $A$ será denotada por $f \Gamma_{A}$.

Se $X$ e $Y$ são conjuntos, então ${ }^{Y} X$ denotará o conjuntos das funções $f: Y \rightarrow X$. 
Em particular ${ }^{\omega} X$ denotará o conjunto das sequências em $X$. Se $f \in{ }^{w} X$ e $A$ é um subconjunto infinito de $\omega$ chamamos a funçào $f \uparrow_{A}$ uma subsequência de $f$. Se existe um ponto $x \in X$ tal que $f(n)=x$ para todo $n \in A$ dizemos que $f$ é constante em $A$, e se o conjunto $\omega \backslash\{n \in A: f(n)=x\}$ é finito, dizemos que $f$ é trivial em $A$.

Um conjunto $A$ é dito transitivo se cada elemento de $A$ é também um subconjunto de $A$. Um conjunto $A$ é um ordinal se é transitivo e bem ordenado por $\in$. Observamos que os elementos de um ordinal são também ordinais. Usaremos as letras gregas $\alpha, \beta, \gamma, \delta, \eta$ para designar ordinais. Se $\alpha$ e $\beta$ são ordinais, utilizamos a expressão $\alpha<\beta$ como equivalente a $\alpha \in \beta$.

Se $X$ é um conjunto de ordinais, então $\sup (X)=\cup X$ e se $X \neq \emptyset, \min (X)=\cap X$. Seja $\alpha$ um ordinal. O sucessor de $\alpha$ é o ordinal $\alpha+1=\alpha \cup\{\alpha\}$. Um ordinal $\alpha$ é chamado ordinal sucessor se existe um ordinal $\beta$ tal que $\alpha=\beta+1$, é chamado ordinal limite se $\alpha \neq 0$ e $\alpha$ não é sucessor.

Dois conjuntos $A$ e $B$ são equipotentes se existe uma função bijetiva $f: A \rightarrow B$. Se $A$ é um conjunto bem ordenado, define-se a cardinalidade $A,|A|$, como o menor ordinal equipotente a $A$. Sob o Axioma da Escolha, todo conjunto admite uma boa ordem e portanto está definida a cardinalidade de todo conjunto. $\omega$ denota o conjunto de todos os números naturais, e é o menor ordinal limite. Dizemos que um conjunto é enumerável se tem cardinalidade menor ou igual a $\omega$. Um cardinal é um ordinal $\alpha$ tal que $\alpha=|\alpha|$. Usaremos as letras gregas $\lambda, \kappa$ para denotar cardinais.

Se $\lambda$ é um cardinal, o cardinal sucessor de $\lambda$ é denotado por $\lambda^{+}$é o menor cardinal estritamente maior que $\lambda$. Todo cardinal infinito é da forma $\omega_{\alpha}$ onde $\alpha$ é um ordinal e $\omega_{\alpha}$ é definido por:
(1) $\omega_{0}=\omega$;
(2) $\omega_{\alpha+1}=\left(\omega_{\alpha}\right)^{+}$ 
(3) Se $\gamma$ é um ordinal limite, $\omega_{\gamma}=\sup \left\{\omega_{\alpha}: \alpha<\gamma\right\}$.

Sejam $\alpha$ e $\beta$ ordinais. Se $f: \alpha \rightarrow \beta$, dizemos que $f$ é cofinal se $i m(f)$ é não limitada em $\beta$. A cofinalidade de $\beta$ é o menor $\alpha$ tal que existe uma função cofinal de $\alpha \operatorname{em} \beta$. Neste caso escrevemos $\operatorname{cf}(\beta)=\alpha$.

Dizemos que um ordinal $\beta$ é regular se $\beta$ é um ordinal limite e $\operatorname{cf}(\beta)=\beta$.

Lema 1.10. (AC) Se $\kappa$ é um cardinal infinito, entâo $\mathrm{r}^{+}$é regular.

Se $X$ é um conjunto denotamos por $\mathcal{P}(X)$ o conjunto das partes de $X$. Pelo teorema de Cantor, temos que $|X|<|\mathcal{P}(X)|$. Entào $\omega<2^{\omega}=|\mathcal{P}(\omega)|$. A Hipótese do Contínuo (CH) é a asserção: $2^{\omega}=\omega_{1}$ e a Hipótese Generalizada do Contínuo (GCH) é a asserçào: $2^{\omega_{\alpha}}=\omega_{\alpha+1}$, para todo ordinal $\alpha$. O cardinal $2^{\omega}$ será denotado por $\mathfrak{c}$.

Lema 1.11. (AC $+\mathrm{GCH})$ Sejam $\kappa, \lambda \geq 2$ cardinais tais que pelo menos um deles é infinito, entäo

(1) $\kappa \leq \lambda$ implica que $\kappa^{\lambda}=\lambda^{+}$.

(2) $\kappa>\lambda \geq \operatorname{cf}(\kappa)$ implica que $\kappa^{\lambda}=\kappa^{+}$.

(3) $\lambda<\mathrm{cf}(\kappa)$ implica que $\kappa^{\lambda}=\kappa$.

Definição 1.12. Uma família de conjuntos $\mathcal{A}$ é chamada um $\Delta$-sistema se existe um conjunto fixo $R$, chamado raiz do $\Delta$-sistema, tal que $A \cap B=R$ para todos os conjuntos $A, B \in \mathcal{A}$, com $A \neq B$.

Se $A$ é um conjunto e $\lambda$ é um cardinal, definimos:

$$
[A]^{\lambda}=\{B \subseteq A:|B|=\lambda\} \text { e }[A]^{<\lambda}=\{B \subseteq A:|B|<\lambda\}
$$

Lema 1.13. (do $\Delta$-sistema) Seja $\kappa$ um cardinal infinito. Seja $\theta>\kappa$ regular e tal que $\forall \alpha<\theta\left(\left|\alpha^{<\kappa}\right|<\theta\right)$. Assuma que $\mathcal{A} \geq \theta$ e que $\forall x \in \mathcal{A}(|x|<\kappa)$, então existe $\mathcal{B} \subset \mathcal{A}$, tal que $|\mathcal{B}|=\theta \in \mathcal{B}$ forma um $\Delta$-sistema. 
Dado um conjunto $X$, um filtro sobre $X$ é uma família $\mathcal{F} \subseteq \mathcal{P}(X)$ que satisfaz:

(i) $\emptyset \notin \mathcal{F}, X \in \mathcal{F}$;

(ii) Se $A, B$ são elementos de $\mathcal{F}$, entào $A \cap B \in \mathcal{F}$;

(iii) Se $A \in \mathcal{F}, B \subseteq X$ e $A \subseteq B$, então $B \in \mathcal{F}$.

Um ultrafiltro sobre $X$ é um filtro sobre $X$ que é maximal no sentido da inclusão. Vamos enumerar algumas propriedades dos ultrafiltros:

(1) Um filtro $\mathcal{F}$ sobre $X$ é um ultrafiltro se e somente se para cada subconjunto $A$ de $X$ tem-se que ou $A \in \mathcal{F}$ ou $X \backslash A \in \mathcal{F}$.

(2) Se $\mathcal{F} \subseteq \mathcal{P}(X)$ satisfaz a propriedade da interseção finita, então existe algum ultrafiltro $\mathcal{U}$ sobre $X$ tal que $\mathcal{F} \subseteq \mathcal{U}$.

(3) Se $\mathcal{H}$ é um ultrafiltro sobre $X$, entào $\bigcap \mathcal{U}$ ou é vazia ou possui um único elemento.

Neste trabalho vamos nos concentrar nos ultrafiltros sobre $\omega$, que vamos denotar com as letras $u$ e $v$. Um ultrafiltro $u$ sobre $\omega$ é dito principal se existir $n \in \omega$ tal que $u=\{A \subseteq \omega: n \in A\} ;$ um ultrafiltro sobre $\omega$ que não é principal é chamado ultrafiltro live. Observamos que pela propriedade (3) acima, se u é um ultrafiltro livre, então $\cap u=\emptyset$. Ultrafiltros livres de $\omega$ não possuem como elementos subconjuntos finitos de $\omega$.

Como falamos no começo do capitulo, neste trabalho utilizamos o sistema axiomático denominado ZFC $(Z F+A C)$. Vamos falar um pouco de consistência e independência. Se $S$ é um conjunto de sentenças e $\varphi$ é uma fórmula, dizemos que $S$ prova $\varphi$ se houver uma dedução formal de $\varphi$ a partir de $S$. Neste caso $\varphi$ é dito um teorema de $S$ e a correçào dos métodos de dedução garante que $\varphi$ é verdadeira em todos os modelos de 
S. Um conjunto de fórmulas é dito consistente se ele não puder provar contradições. Todo conjunto consistente de fórmulas possui um modelo. $\varphi$ é dita consistente com $S$ se $S \cup\{\varphi\}$ for consistente o que é equivalente a dizer que existe algum modelo de $S$ no qual $\varphi$ é verdadeira. Neste caso, se $M$ é um modelo de de $S$ no qual $\varphi$ é verdadeira, escrevemos $(\varphi)^{M} \cdot \varphi$ é dita independente de $S$ se tanto $\varphi$ como a negação de $\varphi$ forem consistentes com $S$. Por exemplo, o Axioma da escolha e a hipótese do contínuo são independentes de ZF.

Neste trabalho "Teorema" significa "teorema de ZFC", nas demosnstrações de resultados de consistê4ncia que utilizem hipóteses ou axiomas adicionais, essas hipóteses serão destacadas.

\section{$1.3 \quad$ Forcing}

Nesta seção pretendemos dar uma idéia muito geral sobre forcing. A referência básica para este tópico é o livro de Kunen [25].

Forcing é uma técnica, criada por Cohen, no ano 1964, para produzir modelos de ZFC que satisfazem diversas propriedades matemáticas.

A idéia é a seguinte: assumindo que existe um modelo trasitivo enumerável M de ZFC, uma ordem parcial $\mathbb{P}$ em $M$ e um $\mathbb{P}$-genérico $\mathbb{G}$ sobre $M$, obter um modelo transitivo e enumerável para ZFC, chamado $M[\mathbb{G}]$ que satisfaz $M \subseteq M[\mathbb{G}], o(M)=o(M[\mathbb{G}])$ e $\mathbb{G} \in \mathrm{M}[\mathbb{G}]$.

Definição 1.14. Uma ordem parcial é uma terna $\langle\mathbb{P}, \leq, 1\rangle$ tal que $\leq$ ordena $\mathbb{P}$ parcialmente e 1 é um elemento máximo de $\mathbb{P}$.

Definição 1.15. $D \subset \mathbb{P}$ é denso em $\mathbb{P}$ se e somente se par cada $p \in \mathbb{P}$, existe $q \in D$ 
tal que $q \leq p$.

Definição 1.16. $\mathbb{G}$ é $\mathbb{P}$-genérico sobre $M$ se satisfaz:

(a) $\forall p, q \in \mathbb{G}$ existe $r \in \mathbb{G}$ tal que $r \leq p$ e $r \leq q$;

(b) $\forall p \in \mathbb{G}, \forall q \in \mathbb{P}$, se $q \leq p$ então $q \in \mathbb{G}$;

(c) para todo denso $D \subset \mathbb{P}$, se $\mathbb{Q} \in M$ entào $\mathbb{G} \cap D \neq \emptyset$.

Lema 1.17. Se $M$ é enumerável $e p \in \mathbb{P}$, então existe $\mathbb{G}$ um $\mathbb{P}$-genérico sobre $M$ tal que $p \in \mathbb{G}$.

É importante destacar que, em geral, um $\mathbb{P}$-genérico sobre $\mathrm{M}$ nào pertence a $\mathrm{M}$. $\mathrm{M}[G]$ é a menor extensão de $\mathrm{M}$ a um modelo transitivo enumerável para ZFC, contendo G. Em geral temos $M \neq M[G]$. Os axiomas adicionais a ZFC que $M[G]$ satisfaz, dependem das propriedades combinatórias da ordem parcial $\mathbb{P}$, em $M$. Isto é, se queremos que uma sentença $\psi$ seja um axioma de $M[\mathbb{G}]$, temos que construir $\mathbb{P}$ com as propriedades combinatórias apropriadas. Os elementos de $\mathbb{P}$ sào condiçòes que dizem alguma coisa a respeito de $\mathbb{G}$ ou de algum objeto que será construido a partir de $\mathbb{G}$. Os moradores de $M$ nào podem construir um $\mathbb{G}$ que seja $\mathbb{P}$-genérico sobre $M$. Eles não sabem o que $\mathbb{G}$ é ou o que os objetos construidos a partir de $\mathbb{G}$ são, mas tem nomes para eles. Cada elemento de $M[\mathbb{G}]$ tem um nome em $M$. Este nome nos diz como este elemento foi construido a partir de $\mathbb{G}$. Os moradores de $\mathrm{M}$ entendem um nome, $\tau$ para um objeto de $M$, mas não sabem o que este objeto (chamado $\tau_{\mathbb{G}}$ ) é, porque isso depende de $\mathbb{G}$ que não está em $M$.

Definição 1.18. $\tau$ é um $\mathbb{P}$-nome se $\tau$ é uma relação e para todo par $\langle\sigma, p\rangle \in \tau, \sigma$ é um $\mathbb{P}$-nome e $p \in \mathbb{P}$.

Definição 1.19. $V^{\mathbb{P}}$ é a classe dos $\mathbb{P}$-nomes. Se M é um modelo transitivo de ZFC e $\mathbb{P} \in \mathrm{M}, \mathrm{M}^{\mathbb{P}}=\mathrm{V}^{\mathbb{P}} \cap \mathrm{M}$. 
Definição 1.20. $\operatorname{val}(\tau, \mathbb{G})=\{\operatorname{val}(\sigma, \mathbb{G}): \exists p \in \mathbb{G}(\langle\sigma, p\rangle \in \tau)\}$. Também escrevemos $\tau_{\mathbb{G}}$ para $\operatorname{val}(\tau, \mathbb{G})$.

Definição 1.21. Se $M$ é um modelo transitivo de $Z F C, \mathbb{P} \in M$ e $\mathbb{G} \subset \mathbb{P}$, então

$$
M[\mathbb{G}]=\left\{\tau_{\mathbb{G}}: \tau \in M^{\mathbb{P}}\right\}
$$

Se $\mathbb{G} \notin M, \operatorname{val}(\tau, \mathbb{G})$ nào significa nada para $M$. Os moradores de $\mathrm{M}$ podem construir uma linguagem de forcing, na qual uma sentença $\psi$ da linguagem usa nomes em $M^{\mathbb{P}}$ para dizer alguma coisa sobre $\mathrm{M}[\mathbb{G}]$. O valor de $\psi$ em $\mathrm{M}[\mathbb{G}]$, se ela é verdadeira ou falsa, depende de $\mathbb{G}$.

Escrevemos $p \Vdash \psi$ para dizer que se $\mathbb{G}$ é um $\mathbb{P}$-genérico sobre $\mathrm{M}$ e $p \in \mathbb{G}$, então $\psi$ é verdadeira em $M[\mathbb{G}]$.

Pode ser decidido dentro de $M$ se $p \Vdash \psi$.

Se $\mathbb{G}$ é $\mathbb{P}$-genérico sobre $M$ e $\psi$ é verdadeira em $M[\mathbb{G}]$, entào para algum $p \in \mathbb{G}, p \Vdash \psi t$.

Definição 1.22. Dois elementos $p, q$ da ordem parcial $\mathbb{P}$ são incompatíveis se nào existe $r \in \mathbb{P}$ tal que $r \leq p$ e $r \leq q$. Uma anticadéia em $\mathbb{P}$ é um subconjunto $A \subset \mathbb{P}$ tal que se $p, q \in A$ e $p \neq q$ então $p$ e $q$ são incompativeis.

Definição 1.23. Seja $\lambda$ é um cardinal infinito de $M$.

(1) Dizemos que a ordem parcial $\mathbb{P}$ é $\lambda$-c.c. se toda anticadéia em $\mathbb{P}$ tem cardinalidade $<\lambda$.

(2) Dizemos que $\mathbb{P}$ é $\lambda$-fechada se, para cada $\gamma<\lambda$ e cada sequência decrescente $\left\{p_{\xi}: \xi<\gamma\right\}$ de elementos de $\mathbb{P}$, temos que existe $q \in \mathbb{P}$ tal que $q \leq p_{\xi}$ para cada $\xi<\gamma$.

Definição 1.24. Seja $\mathbb{P} \in \mathrm{M}$ e seja $\lambda$ um cardinal infinito de $\mathrm{M}$.

(1) $\mathbb{P}$ preserva cardinais $\geq \lambda$ (ou $\leq \lambda$ ) se para todo genérico $\mathbb{G}$ sobre $M$, se $\beta \in o(M)$, 
e $\beta \geq \lambda$ (resp. $\beta \leq \lambda)$, então

$$
(\beta e ́ \text { um cardinal })^{\mathrm{M}} \longleftrightarrow(\beta e ́ \text { um cardinal })^{\mathrm{M}[\mathrm{G}}
$$

(2) $\mathbb{P}$ preserva cofinalidades $\geq \lambda($ ou $\leq \lambda)$ se para todo genérico $\mathbb{G}$ sobre $M$, se $\gamma$ é um ordinal limite em $\mathrm{M}$, e cf $(\gamma)^{\mathrm{M}} \geq \lambda($ resp. $\leq \lambda)$, então

$$
\operatorname{cf}(\gamma)^{\mathrm{M}}=\operatorname{cf}(\gamma)^{\mathrm{M}[\mathrm{G}]}
$$

Lema 1.25. Se $\mathbb{P}$ preserva cofinalidades $\leq \lambda$, então $\mathbb{P}$ preserva cardinais $\leq \lambda$. Se $\mathbb{P}$ preserva cofinalidades $\geq \lambda$ e $\lambda$ é regular em $\mathrm{M}$, então $\mathbb{P}$ preserva cardinais $\geq \lambda$.

Lema 1.26. Se $\mathbb{P} \in \mathrm{M}, \lambda$ é um cardinal de $\mathrm{M}, e \mathbb{P} \dot{\epsilon} \lambda$-c.c. em $\mathrm{M}$, então $\mathbb{P}$ preserva cofinalidades $\geq \lambda$. Portanto, se também $\lambda$ é regular $e m, \mathbb{M}$ preserva cardinais $\geq \lambda$. Teorema 1.27. Sejam $\mathbb{P} \in \mathrm{M}, A, B \in \mathrm{M}, \epsilon, \epsilon m \mathrm{M}, \lambda$ um cardinal, $\mathbb{P} \lambda$-fechado, $\epsilon$ $|A|<\lambda$. Seja $\mathbb{G}$ um $\mathbb{P}$-genérico sobre $M \in$ seja $f \in M[\mathbb{G}]$ com $f: A \rightarrow B$. Então $f \in \mathrm{M}$.

Corolário 1.28. Em $\mathrm{M}$ sejam $\lambda$ um cardinal $\in \mathbb{P} \lambda$-fechado. Entäo $\mathbb{P}$ preserva cofinalidades $\leq \lambda$ e portanto preserva cardinais $\leq \lambda$.

Definição 1.29. Se $\sigma \in V^{\mathbb{P}}$, um nice name para um subconjunto de $\sigma$ é $\tau \in V^{\mathbb{P}}$ da. forma $\cup\left\{\{\pi\} \times A_{\pi}: \pi \in \operatorname{dom}(\sigma)\right\}$, onde $A_{\pi}$ é uma anticadéia em $\mathbb{P}$.

Lema 1.30. Suponhamos que em $\mathrm{M}, \mathbb{P} e ́ \omega \cdots c . c .,|\mathbb{P}|=\kappa \geq \omega, \lambda$ é um cardinal infinito, e $\theta=\kappa^{\lambda}$. Seja $\mathbb{G}$ um $\mathbb{P}$-genérico sobre $\mathrm{M}$. Então $\mathrm{em} \mathrm{M}[\mathbb{G}], 2^{\lambda} \leq \theta$.

\subsection{Topologia Geral}

As referências básicas para os conceitos topológicos são o livro de Engelking [13] e as Notas de aula do curso de Topologia Geral da pos-graduação do IME-USP [33]. 
Um espaço topológico é um par $\langle X, \tau\rangle$, onde $X$ é o conjunto suporte e $\tau$ a topologia definida no conjunto $X$. Em geral vamos nos referir ao espaço $\langle X, \tau\rangle$ por espaço topológico $X$ ou espaço $X$.

Assumiremos conhecidos os conceitos básicos da Topologia Geral, como por exemplo : conjunto aberto, conjunto fechado, vizinhança, base para o espaço, base para $u$ ponto, subconjunto $G_{\delta}$, fecho de um conjunto, axiomas de enumerabilidade e separação.

Se $\langle X, \tau\rangle$ é um espaço topológico, e $Y$ é um subconjunto de $X$, entào

$$
\sigma=\{Y \cap U: U \in \tau\}
$$

define uma topologia para $Y$ chamada topologia induzida por $X$ ou topologia de subespaço de $X$.

Se $\left\{X_{s}: s \in S\right\}$ é uma familia de conjuntos, o produto cartesiano desta família é o conjunto de todas as funçōes $f: S \rightarrow \bigcup_{s \in S} X_{s}$ tal que para cada $s \in S$ temos $f(s) \in X_{s}$. Dado $t \in S$, a funçào $\pi_{t}: \prod_{s \in S} X_{s} \rightarrow X_{t}$ tal que $\pi_{t}(f)=f(t)$ é chamada de projeção de $\prod_{s \in S} X_{s}$ na coordenada $t$. A topologia gerada pe la base

$$
\begin{aligned}
& \left\{\pi_{s_{1}}^{-1}\left(U_{s_{1}}\right) \cap \ldots \cap \pi_{s_{k}}^{-1}\left(U_{s_{k}}\right): s_{1} \ldots s_{k} \in S \text { e } U_{s_{k}} \text { é aberto de } X_{s_{3}}\right\}= \\
= & \left\{\prod_{s \in S} U_{s}: U_{s} \text { é aberto de } X_{s} \text { e }\left\{s \in S: U_{s} \neq X_{s} \text { é finito }\right\}\right\}
\end{aligned}
$$

é o produto de Tychonoff da família $\left\{X_{s}: s \in S\right\}$ e é denotado por $\prod_{s \in S} X_{s}$. Sempre que falemos de produto de espaços estaremos nos referindo a esta topologia. 
Se $\left\{X_{s}: s \in S\right\}$ é uma família de conjuntos, e $Y$ é um conjunto, e se para cada $s \in S f_{s}: Y \rightarrow X_{s}$ é uma função, a função diagonal definida pela família $\left\{f_{s}: s \in S\right\}$ é a função $\triangle_{s \in S} f_{s}: Y \rightarrow \prod_{s \in S} X_{s}$ dada por $\triangle_{s \in S} f_{s}(y)=\left(f_{s}(y)\right)_{s \in S}, \forall y \in Y$.

Seja $X$ um espaço topológico e $A \subseteq X$. Dizemos que $x \in X$ é ponto de acumulação de $A$ se para cada vizinhança $U$ de $x$ em $X, A \cap(U \backslash\{x\}) \neq \emptyset$. Se $A$ é infinito dizemos que $x$ é ponto de acumulação completo de $A$ se para cada vizinhança $U$ de $x$, $|U \cap A|=|A|$. Se $X$ é $T_{1}$ e $A \subseteq X$, então $x \in X$ é ponto de acumulação de $A$ se, e somente se, para cada vizinhança $U$ de $x$ em $X, A \cap X$ é infinito.

Seja $X$ um espaço topológico e seja $f: \omega \rightarrow X$ uma sequência. Dizemos que $f$ converge para um ponto $x$ se para cada vizinhança aberta $U$ de $x$ em $X$,

$$
\omega \backslash\{n<\omega: f(n) \in U\} \text { é finito . }
$$

Dizemos que $x$ é um ponto de acumulação (cluster point) da sequência $f$ se para cada. vizinhança $U$ de $x$ em $X,\{n<\omega: f(n) \in U\}$ é infinito.

Un espaço topológico $X$ é compacto se todo recobrimento aberto de $X$ possui um sub-recobrimento finito.

Teorema 1.31 (Teorema de Tychonoff). Seja $\left\{X_{i}: i \in I\right\}$ uma família de espaços topológicos compactos nào-vazios. Então o produto de Tychonoff $\prod_{i \in I} X_{i}$ é compacto.

Um espaço topológico $X$ é enumeravelmente compacto se toda cobertura aberta enumerável de $X$ possui um sub-recobrimento finito.

Teorema 1.32. Para $X$ um espaço topológico $T_{1}$ sâo equivalentes:

(i) $X$ é enumeravelmente compacto; 
(ii) toda sequência em $X$ possui ponto dè acumulação;

(iii) todo subconjunto infinito enumerável de $X$ possui ponto de acumulação;

(iv) X não tem um subespaço enumerável infinito que seja discreto e fechado.

Toda função contínua a valores reais definida num espaço enumeravelmente compacto $X$ é limitada.

Um espaço topológico $X$ é pseudocompacto se toda funçào a valores reais, contínua definida em $X$ é limitada.

Das definições segue que

$$
\text { compacto } \Rightarrow \text { enumeravelmente compacto } \Rightarrow \text { pseudocompacto, }
$$

mas as recíprocas não valem.

Vamos ver um exemplo de espaço pseudocompacto que näo é enumeravelmente compacto. Seja $X$ um conjunto infinito. Fixamos um ponto $p \in X$ e declaramos $U \subseteq X$ aberto se $p \in U$. Observamos que $X$ é $T_{0}$ mas não é $T_{1}$ pois se $x \in X, x \neq p$, toda vizinhança aberta de $x$ contém também $p$. Se f é uma sequência em $X$, os únicos candidatos a pontos de acumulação de $f$ são os pontos $x \in X$ tais que $\{n<\omega: f(n)=x\}$ é infinito. $X$ é pseudocompacto pois toda função a valores reais contínua definida em $X$ é a constante. $X$ não é enumeravelmente compacto pois nenhuma sequência injetora em $X$ possui ponto de acumulação.

Considerando o teorema de Tychonoff para espaços compactos parece natural se perguntar se o produto de espaços enumeravelmente compactos é também enumeravelmente compacto. A resposta é não, de fato também não é verdade para espaços pseudocompactos. 
Falaremos mais dos espaços enumeravelmente compactos depois de apresentarmos o espaço $\beta \omega$.

\section{$1.5 \beta \omega, \mathrm{P}-$ pontos e ultrafiltros seletivos}

$\beta \omega$ é o compactificado de Stone-Čech do espaço discreto $\omega$. Vamos considerar $\beta \omega$ como o espaço que tem por suporte o conjunto de todos os ultrafiltros sobre $\omega$ e a topologia dada pela base de abertos-fechados $\{V(A): A \subseteq \omega\}$, onde

$$
V(A)=\{u: u \text { é um ultrafiltro sobre } \omega \text { e } A \in u\}
$$

Neste espaço w é homeomorfo ao subespaço denso formado pelos ultrafiltros principais. O espaço $\beta \omega \backslash \omega$ é denotado por $\omega^{*}$.

Todo subconjunto infinito de $\beta \omega$ possui $2^{r}$ pontos de acumulação, portanto as únicas sequências convergentes em $\beta \omega$ são as triviais.

Para cada subconjunto infinito $V$ de $\omega$, o fecho de $V$ em $\beta \omega$ é homeomorfo a $\beta \omega$. Se $f: \omega \rightarrow \omega$ é uma função, então a funçào $\bar{f}: \beta \omega \rightarrow \beta \omega$ dada por

$$
\bar{f}(u)=\left\{A \subseteq \omega: f^{-1}(A) \in u\right\}
$$

é chamada a extensão de Stone-Cech de $f$.

Sobre os elementos de $\beta \omega$ se define uma pre-ordem (propriedades reflexiva e transitiva), chamada ordem de Rudin-Keisler, denotada por $\leq_{R K}$. Dados dois ultrafiltros $u, v$ sobre $\omega$ dizemos que

$$
u \leq_{R K} v \Longleftrightarrow \text { existe } f \in{ }^{\omega} \omega \text { tal que } u=\bar{f}(v)
$$

Um ultrafiltro $u \in \omega^{*}$ é dito minimal na ordem de Rudin-Keisler se não existe $v \in \omega^{*}$ tal que $v \leq_{R K} u$ e $v \not \equiv u$. Então dois ultrafiltros minimais na ordem de 
Rudin-Keisler ou são equivalentes ou são incomparáveis.

Um ultrafiltro livre $u$ é chamado $P$-ponto se para cada partiçäo $\left\{A_{n}: n \in \omega\right\}$ de $\omega$ tal que $A_{n} \notin u$ para todo $n$, existe $X \in u$ tal que

$$
X \cap A_{n} \text { é finito, } \forall n \in \omega
$$

Existem ultrafiltros que não são $\mathrm{P}$-pontos. Por exemplo, seja $\mathcal{F}$ o seguinte filtro sobre $\omega: X \in \mathcal{F}$ se o somente se $A_{n} \backslash\left(X \cap A_{n}\right)$ é finito para todos exceto um número finito de $n$. Se $u$ é um ultrafiltro estendendo $\mathcal{F}$, então $u$ não é um $\mathrm{P}$-ponto. A existência. de P-pontos é independente de ZFC. Existem $2^{c} \mathrm{P}$-pontos sob CH ou MA ( Axioma. de Martin) e Shellah mostrou que existem modelos de ZFC sem $\mathrm{P}$-pontos.

Um ultrafiltro livie $u$ é chamado seletivo se para cada funçào $f: \omega \rightarrow \omega$ existe $A \in u$ tal que $f l_{A}$ é ou constante ou injetora. Como no caso dos P-pontos, sob CH ou MA existem $2^{\mathfrak{r}}$ ultrafiltros seletivos incomparáveis. Da proposiçào seguinte se deduz facilmente que ultrafiltros seletivos sào P-pontos. Portanto a existência de ultrafiltros seletivos é independente de $\mathrm{ZFC}$.

Proposição 1.33. Para u um ultrafiltro livre de w são equivalentes:

(a) u é minimal na ordem de Rudin-Keisler;

(b) ué seletivo;

(c) para cada partiçâo $\left\{P_{k}: k \in \omega\right\}$ de $\omega$, ou existe $k \in \omega$ tal que $P_{k} \in p$ ou existe $A \in u$ tal que $\left|A \cap P_{k}\right| \leq 1 \forall k \in \omega$;

(d) toda coloração $F:[\omega]^{2} \rightarrow\{0,1\}$ possui um conjunto homogêneo $A \in u$.

Os leitores interessados podem ver a demonstração desta proposição no Anexo A deste trabalho. 


\subsection{Mais sobre os espaços enumeravelmente com- pactos}

J. Novák em 1953 e H. Teresaka en 1952 obtiveram dois espaços enumeravelmente compactos $X$ e $Y$ tais que

(i) $\omega \subset X \subset \beta \omega$ e $\omega \subset Y \subset \beta \omega$;

(ii) $X \cap Y=\omega$.

O produto $X \times Y$ não é enumeravelmente compacto: O conjunto $H=\{(n, n): n \in \omega\}$ nào possui ponto de acumulaçào em $X \times Y$. Para ver que isto é verdade basta considerar a diagonal $\Delta=\{(u, u): u \in \beta \omega\}$ de $\beta \omega \times \beta \omega$. Como $\beta \omega \times \beta \omega$ é um espaço $T_{2}$ a diagonal é fechada e portanto $\bar{H} \subset \Delta$. Então se $(x, y)$ é ponto de acumulação de $H$ em $X \times Y$, temos que $x=y$ o que nào possivel por (ii).

Há varios outros exemplos:

(1) (Nóvak, 1952) Existem espaços $X$ e $Y$ tais que toda sequência em $X$ e toda sequência em $Y$ tem $2^{i}$ pontos de acumulaçào, e $X \times Y$ nào é enumeravelmente compacto.

(2) [van Dowen, [11]] Sob MA existem dois grupos enumeravelmente compactos $G$ e $H$ tais que $G \times H$ não é enumeravelmente compacto.

(3) (Frólik, 1967) Existe um espaço enumeravelmente compacto $X$ tal que para todo $n<\omega X^{n}$ é enumeravelmente compacto, mas $X^{\omega}$ não é enumeravelmente compacto.

Ginsburg e Saks [16] mostraram que $\prod_{i \in I} X_{i}$ é enumeravelmente compacto se e somente se $\prod_{i \in J} X_{i}$ é enumeravelmente compacto para cada $J \subseteq I$ tal que $|J| \leq 2^{i}$. 
Seja $u \in \omega^{*}$ e seja $f: \omega \rightarrow X$ uma sequência num espaço $X$. Dizemos que um ponto $x \in X$ é o $u$-limite de $f$ e denotado $x=u-\lim f$ se para cada vinzinhança $U$ de $x,\{n \in \omega: f(n) \in U\} \in u$. Como um ultrafiltro não contém conjuntos disjuntos, uma sequência (num espaço $T_{2}$ ) pode ter no máximo um $u$-limite. Portanto o $u$-limite de uma sequência se existe é único. Observamos também que se $x$ é $u$-limite de uma sequência é também ponto de acumulação da sequência pois ultrafiltros livres só contém conjuntos infinitos.

Lema 1.34. Um ponto $x$ é ponto de acumulaçào de uma sequência f se e somente se existe $u \in \omega^{*}$ tal que $x=u-\lim f$.

Lema 1.35. Um ponto $x \in \prod_{i \in I} X_{i}$ é u-limite da sequência $f: \omega \rightarrow \prod_{i \in I} X_{i}$ se $\varepsilon$ somente se $\pi_{i}(x) \in X_{i}$ é $u$-limite de $\left\{\pi_{i}(f(n)): n \in \omega\right\} \subseteq X_{i}$ para todo $i \in I$, onde $\pi_{I}$ é a projeção de $\prod_{i \in I} X_{i}$ em $X_{i}$.

A demonstraçào do seguinte teorema pode ser lida em [34]

Teorema 1.36. Seja $\left\{X_{\alpha}: a \in I\right\}$ uma família de espaços topológicos. Então o espaço produto $X=\prod\left\{X_{\alpha}: \alpha \in I\right\}$ é enumeravelmente compacto se $\epsilon$ somente se para cada sequência $f: \omega \rightarrow X$, existe $u \in \omega^{*}$ tal que $\pi_{\alpha} \circ f$ possui u-limite em $X_{\alpha}$ para cada $\alpha \in I$.

Já vimos que num espaço enumeravelmente compacto, toda sequência tem $u$-limite em $X$ para algum ultrafiltro livre $u$, mas este ultrafiltro pode ser diferente para cada. sequência.

Seja $u \in \omega^{*}$. Um espaço $X$ é dito $u$-compacto se toda sequência em $X$ possui $u$-limite em $X$. Segue da definição que todo espaço $u$-compacto é enumeravelmente 
compacto. Do teorema 1.36 temos que O produto de espaços $u$-compactos é também $u$-compacto.

Teorema 1.37. Para um espaço $X$ sâo equivalentes:

(i) $X^{r} \dot{e}$ enumeravelmente compacto para todo cardinal $\kappa$;

(ii) $X^{2^{c}}$ é enumeravelmente compacto;

(iii) Existe $u \in \omega^{*}$ tal que $X$ é u-compacto

\subsection{Grupos topológicos}

Os conceitos fundamentais referentes a grupos topológicos podem ser encontrados no trabalho de Comfort [2] e no livro de Hewitt e Ross [20].

Um grupo topológico é um par $\langle G, \tau\rangle$ para o qual

(i) $\langle G, \tau\rangle$ é um espaşo topológico,

(ii) G'é um grupo, e

(iii) A funçào de $G \times G \mathrm{em} G$ definida por $\langle a, b\rangle \rightarrow a b^{-1}$ é contínua.

A seguinte propriedade, bem conhecida, dos grupos topológicos é devida independentemente a Pontrjagin e a Kakutani:

Todo grupo topológico $T_{0}$ é um espaço Hausdorff, completamente regular.

O teorema seguinte é a classificação dada por Harrison e Hulanicki dos grupos abelianos que admitem uma topologia de grupo.

Teorema 1.38. (i) Seja $G$ um grupo abeliano $\epsilon$

$$
G=D(G) \oplus R(G)
$$


sua decomposição na parte divisível $D(G)$ e na parte reduzida $R(G) \cong G / D(G)$. (um grupo é reduzido se não contém subgrupos próprios divisíveis). Então $G$ admite uma topologia de grupo compacto se e somente se $D(G)$ e $R(G)$ admitem ambos uma topologia de grupo compacto.

(ii) Um grupo abeliano divisivel $G$ admite uma topologia de grupo compacto se $\epsilon$ somente se $r_{0}(G)=2^{\sigma}$ para algum cardinal infinito $\sigma$, e cada $r_{p}(G)$ é ou finito ou $2^{\sigma_{p}}$ para algum cardinal $\sigma_{p} \leq \sigma$.

(iii) Um grupo abeliano reduzido $G$ admite uma topologia de grupo compacto se $e$ somente se

$$
G=\prod\left\{\mathbb{Z}_{p}^{\beta_{p}}: p \text { é primo }\right\} \oplus \prod\left\{\mathbb{Z}\left(p^{n}\right)^{\alpha_{n}}: n \in \omega\right\}
$$

onde $\mathbb{Z}_{p}$ é o grupo dos inteiros p-ádicos.

(iv) Se $G$ é um grupo topológico abeliano compacto, entäo ou $r(G)=0$ ou $r(G)=2^{\sigma}$ para algum cardinal infinito $\sigma$.

Um grupo topológico $G=\langle G, \tau\rangle$ é totalmente limitado se para toda vizinhança $U \in \mathcal{N}(0)$ existe um subconjunto finito $F$ de $G$ tal que $G \subseteq F U$. A importancia destes grupos segue da seguinte caracterização:

um grupo topológico é totalmente limitado se e somente se ele é um subgrupo denso de um grupo compacto.

Todo grupo topológico pseudocompacto é totalmente limitado.

$\mathrm{Na}$ construçào de exemplos de grupos pseudocompactos a seguinte propriedade é de grande utilidade.

Seja $X$ um espaço topológico. Um subconjunto $Y$ de $X$ é $G_{\delta}$-denso em $X$ se $Y$ intercepta todo subconjunto $G_{\delta}$ näo vazio de $X$.

Teorema 1.39. Dado um grupo compacto $G$, um subgrupo denso $H$ de $G$ é pseudocompacto se e somente se $H$ é $G_{\delta}$-denso em $G$. 
Teorema 1.40 (Comfort e Robertson [5]). Seja G um grupo topológico pseudocompacto não discreto, então $|G| \geq \mathfrak{c}$.

Vemos que a pseudocompacidade em grupos topológicos é preservada pelo produto, o que não acontece em geral com espaços pseudocompactos:

Teorema 1.41 (Comfort e Ross [6]). Seja $\left\{G_{i}: i \in I\right\}$ um conjunlo de grupos topológicos pseudocompactos e seja $G=\prod_{i \in I} G_{i}$. Então $G$ é pseudocompacto.

O mesmo não pode se dizer dos grupos enumeravelmente compactos:

Teorema 1.42 (van Dowen [11]). Assuma MA. Existem grupos topológicos enumeravelmente compactos $G$ e $H$, subgrupos de $2^{i}$, tais que $|G \cap H|=\omega$. Então $G \times H$ por conter a diagonal

$$
\Delta=\{(x, y) \in G \times H: x=y\}=\{(x, x): x \in G \cap H\}
$$

como subgrupo enumeravel infinito e fechado, näo é enumeravelmente compacto. 


\section{Capítulo 2}

\section{Ultrafiltros seletivos e Grupos de}

\section{torção $u$ - compactos}

Neste capítulo vamos ver que sob a existência de um ultrafiltro seletivo $u$, é possivel estabelecer condições suficientes para que um grupo abeliano de torção admita uma topologia de grupo topológico u-compacta. Estas condições tem algumas conseqüências importantes:

* nos permitem classificar, sob a existência de um ultrafiltro seletivo, os grupos abelianos de torção de cardinalidade $\mathfrak{c}$ que admitem uma topologia de grupo topológico enumeravelmente compacta e sem seqüências convergentes;

** nos permitem classificar, sob GCH, todos os grupos abelianos de torção que admitem uma topologia de grupo enumeravelmente compacta e sem seqüências não triviais convergentes;

*** nos fornecem o primeiro exemplo de grupo topológico enumeravelmente compacto sem seqüencias convergentes de cardinalidade maior que $2^{c}$, e o primeiro exemplo obtido sem usar forcing de um grupo topológico enumeravelmente compacto sem 
seqüências convergentes e de cardinalidade maior que $\mathfrak{c}$.

\subsection{Seqüências e ultraprodutos}

Como ferramenta fundamental para esta construção vamos precisar de um caso simples de ultraproduto, que detalhamos a seguir.

Seja $u$ um ultrafiltro livre sobre $\omega$, e seja $G$ um grupo abeliano de ordem finita $n$.

Definição 2.1. Se $f$ e $g$ são seqüências em $G$, dizemos que $f$ é $u$-equivalente a $g$, e denotamos $f \equiv_{u} g$ se $\{m \in \omega: f(m)=g(m)\} \in u$.

É imediato que $\equiv_{u}$ é uma relação de equivalência. Para cada $f \in{ }^{\omega} G$, denotamos por $[f]_{u}$ a $\equiv{ }_{u}$-classe de equivalencia de $f$ e denotamos por ${ }^{\omega} G / u$ o conjunto das $\equiv$-classes de equivalência de ${ }^{\omega} G$. Isto é ${ }^{w} G / u=\left\{[f]_{u}: f \in{ }^{\omega} G\right\}$.

Se definimos $[f]_{u}+[g]_{u}=[f+g]_{u}$, entào $\left({ }^{\omega} G / u,+\right)$ é um grupo abeliano de ordem limitada. Portanto, ${ }^{\omega} G / u$ é soma direta de grupos cíclicos e ${ }^{\omega} G / u$ possui uma base. Vamos ver o que acontece com este ultraproduto no caso em que o ultrafiltro ué seletivo. Se $f$ é uma sequência em $G$, e $u$ é um ultrafiltro seletivo sobre $\omega$, temos que existe $A \in u$ tal que $f \Gamma_{A}$ é constante ou $f \Gamma_{A}$ é injetora. Então, se $u$ é seletivo, ${ }^{w} G / u=\left\{[f]_{u}: f\right.$ é injetora $\} \cup\left\{[\vec{x}]_{u}: x \in G\right\}$, onde $\vec{x}$ denota a seqüência constante $x$.

Proposição 2.2. $A=\left\{[\vec{x}]_{u}: x \in G\right\}$ é um subgrupo puro de ${ }^{\omega} G / u$.

Demonstração: É claro que $A$ é subgrupo de ${ }^{\omega} G / u$. Vamos verificar que $A$ é puro. Sejam $n \in \mathbb{Z}$ e $[\vec{y}]_{u} \in A$ e suponhamos que existe $[f]_{u} \in{ }^{\omega} G / u$ tal que $n[f]_{u}=[\vec{y}]_{u}$. Então $\{m \in \omega: n . f(m)=y\} \in u$. Em particular, existe $x \in G$ tal que $n x=y$. Logo 
$n[\vec{x}]_{u}=[\vec{y}]_{u}$ e $[\vec{x}]_{u} \in A$.

Segue desta proposição que se $\left\{x_{\xi}: \xi \in \kappa\right\}$ é uma base para $G$ podemos completar $\left\{\left[\vec{x}_{\xi}\right]_{u}: \xi<\kappa\right\}$ com elementos de $\left\{[f]_{u}: f\right.$ é injetora $\}$ para obtermos uma base de ${ }^{\omega} G / u$.

Proposição 2.3. Seja o grupo $\mathbb{Z}(n)^{(\alpha)}$, onde $n$ é um inteiro positivo $\epsilon \alpha$ é um ordinal qualquer. Se $f \in{ }^{\omega}\left(\mathbb{Z}(n)^{(\alpha)}\right)$, entâo existe $g \in{ }^{\omega}\left(\mathbb{Z}(n)^{(\alpha)}\right)$ tal que ord $(g(m))=n$, $\forall m \in \omega \in[f]_{u} \in\left\langle[g]_{u}\right\rangle$.

Demonstração: Seja $\left\{x_{\xi}: \xi<\alpha\right\}$ uma base para $\mathbb{Z}(n)^{(\alpha)}$ e seja $f_{0}$ uma seqüência em $\mathbb{Z}(n)^{(\alpha)}$, com

$$
f_{0}(m)=a_{1}^{m} \xi_{1}^{m}+\ldots+a_{s_{m}}^{m} \xi_{s_{m}}^{m} .
$$

Se $\left\{m \in \omega: \operatorname{ord}\left(f_{0}(m)\right)=n\right\} \in u$, basta considerar qualquer sequiència $g$ que seja $u$-equivalente a $f_{0}$ e cujos elementos sejam todos de ordem $n$. Se não, existe $d_{0}$ divisor próprio de $n$ tal que $\left\{m \in \omega: \operatorname{ord}\left(f_{0}(m)\right)=d_{0}\right\} \in u$. Vamos assumir que $\operatorname{ord}\left(f_{0}(m)\right)=d_{0} \forall m \in \omega$. Temos entào que

$$
d_{0}\left(a_{1}^{m} \xi_{1}^{n}+\ldots+a_{s_{m}}^{m} \xi_{s_{m}}\right)=0, \forall m \in \omega
$$

Como os $\xi_{i}^{m}$ são elementos de uma base, segue que $n \mid d_{0} . a_{i}^{m}, \forall i=1, \ldots, s_{m}$. Se $n=d_{0} . t$ temos que $t \mid a_{i}^{m} \forall i=1, \ldots, s_{m}$. Podemos entào definir $f_{1} \in{ }^{\omega}\left(\mathbb{Z}(n)^{(\alpha)}\right)$ por

$$
f_{1}(m)=\frac{a_{1}^{m}}{t} x_{\xi_{1}^{m}}+\ldots+\frac{a_{s_{m}}^{m}}{t} x_{\xi_{m}^{m}}, \text { para cada } m \in \omega .
$$

Segue que $f_{0}(m)=t . f_{1}(m), \forall m \in \omega$ e portanto $f_{0} \in\left\langle\left[f_{1}\right]_{u}\right\rangle$ e $\operatorname{ord}\left(f_{1}(m)\right)=n$, $\forall m \in \omega$.

Desta proposição se deduz que todos os elementos de uma base para ${ }^{\omega}\left(\mathbb{Z}(n)^{(\alpha)}\right) / u$ tem ordem $n$. 


\subsection{Uma topologia $u$-compacta para $\mathbb{Z}(r)^{(\mathfrak{c})}$.}

Em [15], S. Garcia-Ferreira, A.H. Tomita e S. Watson apresentaram o primeiro exemplo de um grupo topológico enumeravelmente compacto sem seqüências convergentes obtido sem usar alguma versão do Axioma de Martin. Assumindo a existência de um ultrafiltro seletivo $u$ sobre $\omega$, deram a $\mathbb{Z}(2)^{(\mathfrak{c})}$ uma topologia de grupo topológico $u-$ compacta.

Nesta seçào apresentamos uma construção similar à de [15], para dar a $\mathbb{Z}(r)^{(\mathfrak{c})}$ uma topologia $u$-compacta, onde $r$ é número primo qualquer. Poderia ser escolhido um número natural qualquer, mas sendo $r$ primo $\mathbb{Z}(r)$ é um espaço vetorial e isto simplifica as coisas.

O objetivo de apresentarmos este caso particular é facilitar a leitura da próxima seçào, que terá notação um pouco mais complicada.

Teorema 2.4. Se triste um ultrafiltro seletivo u sobre $\omega$, então $G=\mathbb{Z}(r)^{(\mathfrak{r})}$ admite uma topologia de grupo topológico u-compacta e sem seqüencias náo triviais convergentes.

Seja $\left\{x_{\alpha}: \alpha<\mathfrak{c}\right\}$ uma base pasa o espaço $\mathbb{Z}(r)^{(\mathfrak{c})}$ e seja

$$
\left\{\left[f_{\xi}\right]_{u}: \xi<\mathfrak{c}\right\} \cup\left\{\left[\vec{x}_{\alpha}\right]_{u}: \alpha<\mathfrak{c}\right\}
$$

uma base para o espaço vetorial ${ }^{\omega}\left(\mathbb{Z}(r)^{(\mathfrak{c})}\right) / u$, tal que $f_{\xi}: \omega \rightarrow \mathbb{Z}(r)^{(\mathfrak{c})}$ é injetora e $\operatorname{supp}\left(f_{\xi}(m)\right) \subseteq \max \{\omega, \xi\}, \forall m \in \omega, \forall \xi<\mathfrak{c}$.

A idéia é construir um homomorfismo injetor $\varphi: G \rightarrow \mathbb{Z}(r)^{\mathrm{c}}$ tal que $\varphi(G)$, com a topologia de subespaço, seja $u$-compacto e sem seqüências não triviais convergentes. Para fazer $\varphi$ injetora vamos tomar conta de um elemento do grupo $G$ de cada vez, 
e para fazer com que $\varphi(G)$ não tenha seqüências convergentes vamos tomar conta de uma seqüência não trivial de cada vez. Mas não é necessário trabalhar com todas as sequências não triviais. A seguir explicamos por que.

Se $g: \omega \rightarrow G$ temos duas possibilidades para ela: ou $g$ é quase constante ou $g$ contém uma subseqüência injetiva. Portanto basta considerarmos as seqüencias injetivas em $G$, isto é, as seqüencias $f$ em $G$ tais que

$$
[f]_{u}=\sum_{j=0}^{k_{f}} a_{j}\left[f_{\xi_{j}}\right]_{u}, \text { onde } a_{j} \in \mathbb{Z}, a_{j} \neq 0(\bmod r), \forall 0 \leq j \leq k_{f} \text {. }
$$

Continuando com a idéia da demonstração, vamos construir homomorfismos $\phi_{f, x}$ para cada par $\{f, x\}$, onde $f$ é uma seqüência injetora e $x \in G$. Este homomorfismo vai ser responsável por fazer com que $\varphi(x)$ seja diferente de zero se $x$ é diferente de zero, e por fazer com que a seqüência $\{\varphi(f(m)): m \in \omega\}$ não seja convergente. É claro que ao mesmo tempo temos que fazer com que toda sequiencia em $\varphi(G)$ possua $u$-limite. Mas vamos ver que para isto é suficiente com que para cada par $\{f, x\}$ a seqüêencia $\left\{\phi_{f, x}\left(f_{\xi}(m)\right): m \in \omega\right\}$ tenha $u$ - limite $\phi_{f, x}\left(x_{\xi}\right)$ em $\phi_{f, x}(G)$, para cada $\xi<\mathfrak{c}$.

Lema 2.5. Seja u um ultrafiltro sobre $\omega$. Se $\varphi: G \rightarrow \mathbb{Z}(r)^{i}$ é um monomorfismo tal que para cada $\xi<\mathfrak{c}, \varphi\left(x_{\xi}\right)=u-\lim _{m \in \omega} \varphi\left(f_{\xi}(m)\right)$, então $\varphi(G)$ é u-compacto como subespaço de $\mathbb{Z}(r)^{\mathfrak{c}}$.

Demonstração: Seja $g$ uma seqüência qualquer em $G$. Escrevemos $[g]_{u}$ como combinação linear dos elementos da base:

$$
[g]_{u}=\sum_{j=0}^{k_{g}} a_{j}\left[f_{\xi_{j}}\right]_{u}+\sum_{s=0}^{h_{g}} c_{s}\left[x_{\alpha_{z}}\right]_{u}
$$


Seja $A=\left\{m \in \omega: g(m)=\sum_{j=0}^{k_{g}} a_{j} \cdot f_{\xi_{j}}(m)+\sum_{s=0}^{h_{g}} c_{s} \cdot x_{\alpha_{s}}\right\}$

Como $\varphi\left(x_{\xi_{j}}\right)=u-\lim _{m \in \omega} \varphi\left(f_{\xi_{j}}(m)\right) \forall 0 \leq j \leq k_{g}$ e $A \in u$, temos que

$$
u-\lim _{m \in \omega} \varphi(g(m))=\sum_{j=0}^{k_{g}} a_{j} \cdot \varphi\left(x_{\xi_{j}}\right)+\sum_{s=0}^{h_{g}} c_{s} \cdot \varphi\left(x_{\alpha_{s}}\right) .
$$

Lema 2.6. Seja $x \in G$ e seja $f \in{ }^{\omega} G$ tal que

$$
[f]_{u}=\sum_{j=0}^{k_{f}} a_{j}\left[f_{\xi_{j}}\right]_{u}, \text { com } a_{j} \in \mathbb{Z} \quad \epsilon \sum_{j=0}^{k_{f}} a_{j}\left[f_{\xi_{j}}\right]_{u} \neq 0 .
$$

Então existem uma seqüência crescente $\left\{b_{i}: i \in \omega\right\} \in u$, e uma seqüência $\left\{E_{i}: i \in \omega\right\}$ de subconjuntos finitos de $\mathfrak{c}$ tais que:

(a) $\operatorname{supp}(x) \cup\left\{\xi_{j}: 0 \leq j \leq k_{f}\right\} \subseteq E_{0}$;

(b) $\omega \subseteq \cup_{i \in \omega} E_{i}$;

(c) $E_{i+1} \supseteq \bigcup_{\xi \in E_{i}} \operatorname{supp}\left(f_{\xi}\left(b_{i}\right)\right) \bigcup E_{i}$;

(d) $\left\{f_{\xi}\left(b_{i}\right): \xi \in E_{i}\right\} \cup\left\{x_{\alpha}: \alpha \in E_{i}\right\}$ é linearmente independente, para todo $i<\omega$;

(e) $f\left(b_{i}\right)=\sum_{j=0}^{k_{f}} a_{j} f_{\xi_{j}}\left(b_{i}\right) \quad \forall i \in \omega$.

Demonstração: Definimos por indução uma família $\left\{F_{k}: k \in \omega\right\}$ de subconjuntos finitos de $\mathfrak{c}$ como segue:

$F_{0}=\operatorname{supp}(x) \bigcup\left\{\xi_{j}: 0 \leq j \leq k_{f}\right\}$,

$F_{k+1}=\bigcup_{\xi \in F_{k}}\left\{\operatorname{supp}\left(f_{\xi}(m)\right), m \leq k\right\} \cup F_{k} \cup k, \forall k \in \omega$.

Sejam, para cada $k \in \omega$

$A_{k}^{0}=\left\{m \in \omega:\left\{f_{\xi}(m): \xi \in F_{k}\right\} \cup\left\{x_{\alpha}: \alpha \in F_{k}\right\}\right.$ é linearmente independente $\}$ e seja $U=\left\{m \in \omega: f(m)=\sum_{j=0}^{k_{f}} a_{j} f_{\xi_{j}}(m)\right\}$ 
Como $\left\{\left[f_{\xi}\right]_{u}: \xi<\mathfrak{c}\right\} \bigcup\left\{\left[\vec{x}_{\alpha}\right]_{u}: \alpha<\mathfrak{c}\right\}$ é uma base do espaço vetorial ${ }^{\omega}\left(\mathbb{Z}(r)^{(\mathfrak{s})}\right) / u$, temos que $A_{k}^{0} \in u, \forall k<\omega$. E como $U \in u$ temos que para cada $k \in \omega, A_{k}=A_{k}^{0} \cap U \in$ $u$.

Como $u$ é $\mathrm{P}$-ponto, existe $A \in u$ tal que $A \backslash A_{k}$ é finito para todo $k<\omega$.

Seja $h: \omega \rightarrow \omega$ uma função crescente tal que $A \backslash A_{k} \subseteq h(k)$, para cada $k \in \omega$.

Pela seletividade de $u$, existe $B \in u$ que satisfaz

$$
B \cap h(1)=\emptyset, \quad B \subseteq A,|B \cap[h(k)+1, h(k+1)]| \leq 1 \forall k \in \omega
$$

Seja $\left\{a_{k}: k \in \omega\right\}$ uma enumeraçào crescente de $B$. Entào $k<a_{k}<a_{m}$ e $a_{k} \in A_{k}$ para todo $k<m<\omega$. Definimos em $[\omega]^{2}$ uma coloração $P_{0}, P_{1}$ por:

$$
\begin{aligned}
& \{a, b\} \in P_{0} \text { se e somente se } a, b \in B, a<b \text { e } a_{k}<m \text { se } a=a_{k} \text { e } b=a_{m}, \\
& \{a, b\} \in P_{1} \text { caso contrário. }
\end{aligned}
$$

De novo pela seletividade de $u$, existe $C \in u, C \subseteq B$ tal que $[C]^{2} \subseteq P_{0}$ ou $[C]^{2} \subseteq P_{1}$. Seja $I \in \omega^{w}$ tal que $C=\left\{a_{k}: k \in I\right\}$ e seja $\left\{i_{m}: m<\omega\right\}$ uma enumeração crescente de $I$. Suponhamos que $[C]^{2} \subseteq P_{1}$, entào $a_{i_{0}} \geq i_{k}, \forall 1 \leq k<\omega$, o que é absurdo. Logo $[C]^{2} \subseteq P_{1}$ e portanto $i_{k}<a_{i_{k}}<i_{k+1}, \forall k<\omega$.

Definimos, para cada $k<\omega, b_{k}=a_{i_{k}}$ e $E_{k}=F_{i_{k}}$. Então

$$
\begin{aligned}
& E_{k} \cup \bigcup_{\xi \in E_{k}} \operatorname{supp}\left(f_{\xi}\left(b_{k}\right)\right)=F_{i_{k}} \cup \bigcup_{\xi \in F_{i_{k}}} \operatorname{supp}\left(f_{\xi}\left(a_{i_{k}}\right)\right) \subseteq \\
\subseteq & F_{i_{k}} \cup \bigcup_{\xi \in F_{i_{k}}}\left\{\operatorname{supp}\left(f_{\xi}(m)\right), m<i_{k+1}\right\} \subseteq F_{i_{k+1}}=E_{k+1} .
\end{aligned}
$$

De $a_{i_{k}} \in A_{k} \quad$ seguem $(\mathrm{d})$ e (f). 
Lema 2.7. Seja u um ultrafiltro seletivo sobre $\omega$. Sejam $x \in G$ ef uma seqüência em $G$ tal que

$$
[f]_{u}=\sum_{j=0}^{k_{f}} a_{j} \cdot\left[f_{\xi_{j}}\right]_{u}
$$

onde $a_{j} \in \mathbb{Z}, e \sum_{j=0}^{k_{f}} a_{j}\left[f_{\xi_{j}}\right]_{u} \neq 0$. Então existe um homomorfismo $\phi_{f, x}: G \rightarrow \mathbb{Z}(n)$ que satisfaz:

1) $\phi_{f, x}(x) \neq 0$ se $x \neq 0$

2) $\left\{m \in \omega: \phi_{f, x}\left(f_{\xi}(m)\right)=\phi_{f, x}\left(x_{\xi}\right)\right\} \in u \quad \forall \xi<\mathfrak{c}$,

3) $\left\{m \in \omega: \phi_{f, x}(f(m))=\sum_{j=0}^{k_{f}} a_{j} . \phi_{f, x}\left(x_{\xi_{j}}\right)+\sum_{s=0}^{h_{f}}+1\right\}$ é infinito.

Demonstração: Observamos primeiro que o item 3) faz sentido pois como $r$ é primo, temos que ord $(f(m))=r$, para todo $m \in \omega$ e portanto $\operatorname{ord}\left(\phi_{f, x}(f(m))\right) \leq \operatorname{ord}(f(m))$. Pelo lema 2.6 existem uma seqüência crescente $\left\{b_{i}: i \in \omega\right\} \in u$ e uma seqüencia $\left\{E_{i}: i \in \omega\right\}$ de subconjuntos finitos de $c$ tais que:

(a) $\operatorname{supp}(x) \cup\left\{\xi_{j}: 0 \leq j \leq k_{f}\right\} \subseteq E_{0}$;

(b) $\omega \subseteq \cup_{i \in \omega} E_{i}$;

(c) $E_{i+1} \supseteq \bigcup_{\xi \in E_{i}} \operatorname{supp}\left(f_{\xi}\left(b_{i}\right)\right) \bigcup E_{i}$;

(d) $\left\{f_{\xi}\left(b_{i}\right): \xi \in E_{i}\right\} \cup\left\{x_{\alpha}: \alpha \in E_{i}\right\}$ é linearmente independente, para todo $i<\omega$;

(e) $f\left(b_{i}\right)=\sum_{j=0}^{k_{j}} a_{j} f_{\xi_{j}}\left(b_{i}\right) \quad \forall i \in \omega$.

Como $\left\{b_{i}: i \in \omega\right\} \in u$ temos que $\left\{b_{2 i}: i \in \omega\right\} \in u$ ou $\left\{b_{2 i+1}: i \in \omega\right\} \in u$. Podemos assumir s.p.g. que $\left\{b_{2 i}: i \in \omega\right\} \in u$.

Definimos primeiro $\phi_{f, x}$ em $\left\{x_{\xi}: \xi \in \bigcup_{i \in \omega} E_{i}\right\}$ por indução.

Para cada $\alpha \in E_{0}$, definimos $\phi_{f, x}\left(x_{\alpha}\right)$ de tal forma que $\phi_{f, x}(x) \neq 0$. 
Suponhamos que para $h \in \omega$, temos definida $\phi_{f, x}$ em $\left\langle\left\{x_{\xi}: \xi \in E_{h}\right\}\right\rangle$ tal que para cada $k<h$,

i) se $k$ é par, então

$\phi_{f, x}\left(f_{\xi}\left(b_{k}\right)\right)=\phi_{f, x}\left(x_{\xi}\right) \quad \forall \xi \in E_{k}$

ii) se $k$ é impar, então

$\sum_{j=0}^{k_{f}} \phi_{j, x}\left(a_{j} \cdot f_{\xi_{j}}\left(b_{k}\right)\right)=\sum_{j=0}^{k_{j}} a_{j} \cdot \phi_{f, x}\left(x_{\xi_{j}}\right)+1$.

Como $\left\{f_{\xi}\left(b_{h}\right): \xi \in E_{h}\right\} \cup\left\{\left\{x_{\alpha}\right\}: \alpha \in E_{h}\right\}$ é linearmente independente, podemos estender $\phi_{f, x}$ a $\left\langle\left\{x_{\alpha}: \alpha \in E_{h+1}\right\}\right\rangle$ satisfazendo:

i) $\phi_{f, x}\left(f_{\xi}\left(b_{h}\right)\right)=\phi_{f, x}\left(x_{\xi}\right) \quad \forall \xi \in E_{h}$, se $h$ é par;

ii) $\sum_{j=0}^{k_{f}} \phi_{f, x}\left(a_{j} . f_{\xi_{j}}\left(b_{h}\right)=\sum_{j=0}^{k_{f}} a_{j} \phi_{f, x}\left(x_{\xi_{j}}\right)+1\right.$, se $h$ é impar.

Temos entào definido o homomorfismo $\phi_{f, x}$ no espaço gerado por $\left\{x_{\alpha}: \alpha \in \bigcup_{i \in \omega} E_{i}\right\}$ e verifica:

$$
\begin{aligned}
& \left\{m \in \omega: \phi_{f, x}\left(f_{\xi}(m)\right)=\phi_{f, x}\left(x_{\xi}\right)\right\} \in u \forall \xi \in \bigcup_{i \in \omega} E_{i}, \mathrm{e} \\
& \left\{m \in \omega: \phi_{f, x}(f(m))=\sum_{j=0}^{k_{j}} a_{j} \phi_{f, x}\left(x_{\xi_{j}}\right)+1\right\} \text { é infinito. }
\end{aligned}
$$

Falta então definirmos $\phi_{f, x}\left(x_{\alpha}\right)$ para cada $\alpha \in \mathfrak{c} \backslash\left(\bigcup_{i \in \omega} E_{i}\right)$. Esta definição vai ser feita por indução transfinita. Seja $\gamma \in \mathfrak{c} \backslash\left(\bigcup_{i \in \omega} E_{i}\right)$ e suponhamos $\phi_{f, x}$ definida em $\left\{x_{\alpha}: \alpha \in \bigcup_{i \in \omega} \gamma \cup E_{i}\right\}$. Sabemos que $\bigcup_{k \in \omega} \operatorname{supp}\left(f_{\gamma}(k)\right) \subseteq \gamma$, portanto podemos definir $\phi_{f, x}$ tal que:

$$
\phi_{f, x}\left(x_{\gamma}\right)=u-\lim _{k \in \omega}\left(\phi_{f, x}\left(f_{\gamma}(k)\right) .\right.
$$


Demonstração do Teorema 2.4: Para cada $x \in G$ e para cada seqüência $f$ de $G$ tal que

$$
[f]_{u}=\sum_{j=0}^{k_{f}} a_{j}\left[f_{\xi_{j}}\right]_{u},
$$

onde $a_{j} \in \mathbb{Z}$ e $\sum_{j=0}^{k_{f}} a_{j} \cdot\left[f_{\xi_{j}}\right]_{u} \neq 0$, seja $\phi_{f, x}$ como no lema 2.7.

Definimos o homomorfismo $\varphi: G \rightarrow \mathbb{Z}(n)^{c}$ por

$$
\varphi(z)=\triangle_{(f, x)} \phi_{f, x}(z)
$$

Vemos que se $x \in G \operatorname{com} x \neq 0$, entào $\varphi(x) \neq 0$ pois $\phi_{f, x}(x) \neq 0$. Portanto o homomorfismo $\varphi$ é injetor e $\varphi(G)$ é um subgrupo de $\mathbb{Z}(n)^{\mathfrak{r}}$ isomorfo a $G$.

Vamos verificar agora que $\varphi(G)$ com a topologia de subespaço de $\mathbb{Z}(n)^{c}$ é $u$-compacto.

Do Lema 2.5 segue que basta verificar que $u-\lim _{k \in \omega} \varphi\left(f_{\xi}(m)\right)=\varphi\left(x_{\xi}\right), \forall \xi<\mathfrak{c}$.

Do Lema 2.7 temos que para cada par $(f, x), u-\lim _{k \in \omega} \phi_{f, x}\left(f_{\xi}(m)\right)=\phi_{f, x}\left(x_{\xi}\right), \forall \xi<\mathfrak{c}$. Portanto $u-\lim _{k \in \omega} \varphi\left(f_{\xi}(m)\right)=\varphi\left(x_{\xi}\right), \forall \xi<c$.

Só falta verificar que $\varphi(G)$ não possui seqüências não triviais convergentes.

Seja $\{\varphi(f(m)): m \in \omega\}$ uma seqüência injetiva, com $[f]_{u}=\sum_{j=0}^{k_{f}} a_{j} \cdot\left[f_{\xi_{j}}\right]_{u}$, onde $a_{j} \in \mathbb{Z}$ e $\sum_{j=0}^{k_{f}} a_{j}\left[f_{\xi_{j}}\right]_{u} \neq 0$. Segue do item 3) do Lema 2.7 que $\left\{m \in \omega: \phi_{f, x}(f(m))=\sum_{j=0}^{k_{f}} a_{j} \phi_{f, x}\left(x_{\xi_{j}}\right)+1\right\}$ é infinito. Mas

$$
u-\lim _{m \in \omega} \varphi(f(m))=u-\lim _{n \in \omega} \phi_{f, x}(f(m))=\sum_{j=0}^{k_{f}} a_{j} \cdot \phi_{f, x}\left(x_{\xi_{j}}\right) .
$$

Portanto a seqüência $\{\varphi(f(m)): m \in \omega\}$ não converge. 


\subsection{Grupos de torção $u$-compactos}

Segue o enunciado da condição suficiente da qual falamos na introdução deste capítulo.

Teorema 2.8. Seja G um grupo abeliano de torçấo limitada tal que para cada componente primária $G_{r}$ de $G$ e cada invariante de Ulm-Kaplansky infinita $\alpha_{r k}$ de $G_{r}$, correspondente a $\mathbb{Z}\left(r^{k}\right)$, existe um inteiro $k^{\prime} \geq k$ tal que a invariante $a_{r k^{\prime}}$ satisfaz $\alpha_{r k} \leq \alpha_{r k^{\prime}}$ e $\left(\alpha_{r k^{\prime}}\right)^{\omega}=\alpha_{r k^{\prime}}$. Se existe um ultrafiltro seletivo $u$ sobre $\omega$, então $G$ admite uma topologia de grupo topológico u-compacta e sem seqüências nào triviais convergentes.

Por que precisamos impor esta condição ao grupo $G$ ?

Sabemos que se $n$ é a ordem do grupo $G$ e $r_{1}^{t_{1}} \ldots r_{s}^{t_{s}}$ é a decomposição em fatores

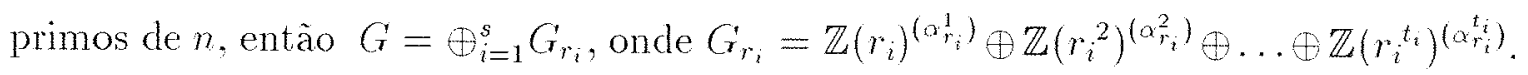
Vamos fixar um $i$, com $1 \leq i \leq s$ e seja $r=r_{i}$ e $t=t_{i}$. Suponhamos que $1 \leq k<t$, que $\alpha_{r}^{k}$ é um cardinal infinito e que $\left(\alpha_{r}^{k}\right)^{\omega}>\alpha_{r}^{k}$. Entào temos mais sequêencias em $\mathbb{Z}\left(r^{k}\right)^{\left(\alpha_{r}^{k}\right)}$ do que possiveis $u$-limites para elas (pois vamos ter $u$-limites diferentes para seqüências diferentes). Portanto vamos precisar de " $u$-limites" para seqüências de $\mathbb{Z}\left(r^{k}\right)^{\left(\alpha_{r}^{k}\right)}$, fora de $\mathbb{Z}\left(r^{k}\right)^{\left(\alpha_{r}^{k}\right)}$. Por hipótese existe $j, \operatorname{com} k<j \leq t$ tal que $\left(\alpha_{r}^{j}\right)^{\omega}=\alpha_{r}^{j}$ e $\left(\alpha_{r}^{k}\right)^{\omega} \leq \alpha_{r}^{j}$. Como $\mathbb{Z}\left(r^{j}\right)^{\left(\alpha_{r}^{j}\right)} \cong \mathbb{Z}\left(r^{j}\right)^{\left(\alpha_{r}^{j}\right)} \oplus \mathbb{Z}\left(r^{j}\right)^{\left(\alpha_{r}^{j}\right)}$, temos em $\mathbb{Z}\left(r^{j}\right)^{\left(\alpha_{r}^{j}\right)}$ candidatos suficientes a " $u$-limites" para as seqüencias em $\mathbb{Z}\left(r^{k}\right)^{\left(\alpha_{r}^{k}\right)}$ e para as seqüências em $\mathbb{Z}\left(r^{j}\right)^{\left(\alpha_{r}^{j}\right)}$. Isto é, vamos fazer com que

$$
\mathbb{Z}\left(r^{k}\right)^{\left(\alpha_{r}^{k}\right)} \oplus \mathbb{Z}\left(r^{j}\right)^{\left(\alpha_{r}^{j}\right)} \oplus \mathbb{Z}\left(r^{j}\right)^{\left(\alpha_{r}^{j}\right)} \cong \mathbb{Z}\left(r^{k}\right)^{\left(\alpha_{r}^{k}\right)} \oplus \mathbb{Z}\left(r^{j}\right)^{\left(\alpha_{r}^{j}\right)}
$$

seja $u$-compacto.

Como a $u$-compacidade é produtiva, todo outro caso reduz-se ao que acabamos de comentar. 
Vamos simplificar um pouco a notação. Se $G$ é como no enunciado do teorema, e $n$ é a ordem de $G$, então existem um subgrupo finito $F$ de $G$ e um divisor $m>1$ de $n$ tais que $G \cong F \oplus \oplus_{d / m} \mathbb{Z}(d)^{\left(\beta_{d}\right)}$, onde $\beta_{m}$ satisfaz $\beta_{m}{ }^{\omega}=\beta_{m}$. Como o produto de um grupo finito por um grupo $u$-compacto é $u$-compacto, podemos assumir que $F=\{0\}$ e que $m=n$. Também podemos assumir $G=\mathbb{Z}(d)^{\left\{\beta_{d}\right)} \oplus \mathbb{Z}(n)^{\left(\beta_{n}\right)}$ onde $d$ é divisor de $n, \beta_{d}<\beta_{n}$ e $\beta_{d}{ }^{\omega} \leq \beta_{n}$, pela produtividade da $u$-compacidade e porque o conjunto dos divisores de $n$ é finito.

Vamos fixar $\left\{x_{\alpha}: \alpha<\beta_{d}\right\}$ uma base para $\mathbb{Z}(d)^{\left(\beta_{d}\right)}$ e $\left\{x_{\alpha}: \beta_{d} \leq \alpha<\beta_{n}\right\}$ uma base para o grupo $\mathbb{Z}(n)^{\left(\hat{\beta}_{n}\right)}$.

Consideramos agora os ultraprodutos $\omega\left(\mathbb{Z}(d)^{\left(\hat{\beta}_{i}\right)}\right) / u$ e ${ }^{\omega}\left(\mathbb{Z}(n)^{\left(\beta_{n}\right)}\right) / u$, e vamos enumerar a funções injetoras na base de cada um deles.

Como $\beta_{d}{ }^{\omega} \leq \beta_{n}$, podemos assumir (para simplificar a notação) que $\beta_{d}{ }^{\omega}=\beta_{n}$. Seja $\left\{I_{0}, I_{1}\right\}$ uma partição de $\beta_{n} \backslash \beta_{d}$ tal que $\left|I_{0}\right|=\left|I_{1}\right|=\beta_{n}$. Então enumeramos por $\left\{f_{\xi}: \xi \in \beta_{n} \backslash \beta_{d}\right\}$ as funçöes injetoras que satisfazem:

(1) $f_{\xi}: \omega \rightarrow \mathbb{Z}(d)^{\left(\beta_{d}\right)} \forall \xi \in I_{0}$ e $\left\{\left[f_{\xi}\right]_{u}: \xi \in I_{0}\right\} \cup\left\{\left[\vec{x}_{\left.u_{i}\right]_{u}}: \alpha<\beta_{d}\right\}\right.$ é uma base para $\omega\left(\mathbb{Z}(d)^{\left(\beta_{d}\right)}\right) / u$

(2) $f_{\xi}: \omega \rightarrow \mathbb{Z}(n)^{\left(\beta_{n 2}\right)} \forall \xi \in I_{1}$ e $\left\{\left[f_{\xi}\right]_{u}: \xi \in I_{1}\right\} \cup\left\{\left[\vec{x}_{\alpha}\right]_{u}: \beta_{d} \leq \alpha<\beta_{n}\right\}$ é uma base para $^{\omega}\left(\mathbb{Z}(n)^{\left(\beta_{n}\right)}\right) / u$ e $\bigcup_{k<\omega} \operatorname{supp}\left(f_{\xi}(k)\right) \subseteq \xi, \forall \xi \in I_{1}$.

Observamos que:

(i) $\bigcup_{k<\omega} \operatorname{supp}\left(f_{\xi}(k)\right) \subseteq \beta_{d l}, \forall \xi \in I_{0}$

(ii) $\operatorname{ord}\left(f_{\xi}(k)\right)=d, \forall k \in \omega, \forall \xi \in I_{0} \operatorname{eord}\left(f_{\xi}(k)\right)=n, \forall k \in \omega, \forall \xi \in I_{1}$,

(iii) se $x \in G$, então $x=x_{0}+x_{1}$, com $x_{0} \in \mathbb{Z}(d)^{\left(\beta_{d}\right)}$ e $x_{1} \in \mathbb{Z}(n)^{\left(\beta_{n}\right)}$, isto é $\operatorname{supp}(x)=$ 
$\operatorname{supp}\left(x_{0}\right) \cup \operatorname{supp}\left(x_{1}\right)$, onde $\operatorname{supp}\left(x_{0}\right) \in\left[\beta_{d}\right]^{<\omega} \operatorname{esupp}\left(x_{1}\right) \in\left[\beta_{n} \backslash \beta_{d}\right]^{<\omega}$,

(iv) se $f \in G^{\omega}$ então $[f]_{u}=\sum_{j=0}^{k_{f}} a_{j}\left[f_{\xi_{j}}\right]_{u}+\sum_{s=0}^{h_{f}} c_{s}\left[\vec{x}_{\alpha_{s}}\right]_{u}$, onde $a_{j} \in \mathbb{Z}, c_{s} \in \mathbb{Z}$, $\beta_{d} \leq \xi_{j}<\beta_{n}, \alpha_{s}<\beta_{n}$.

A idéia é construir um monomorfismo $\varphi: G \rightarrow \mathbb{Z}(n)^{\beta_{n}}$ tal que $\varphi(G)$ com a topologia de subespaço de $\mathbb{Z}(n)^{\beta_{n}}$ seja $u$-compacto e sem seqüências convergentes. Para conseguir a segunda condição tomamos conta de uma seqüência de cada vez. Vamos construir homomorfismos $\phi_{f, x}: G \rightarrow \mathbb{Z}(n)$, para cada $x \in G$ e cada sequiência injetora $f$ tal que $[f]_{u} \in\left\langle\left\{\left[f_{\xi}\right]_{u}: \beta_{0} \leq \xi<\beta_{n}\right\}\right\rangle$.

Lema 2.9. Seja $x \in G$ e seja $f \in{ }^{\omega} G$ tal que

$$
[f]_{u}=\sum_{j=0}^{k_{f}} a_{j}\left[f_{\xi_{j}}\right]_{u}, \text { com } a_{j} \in \mathbb{Z} \quad \epsilon \sum_{j=0}^{k_{f}} a_{j}\left[f_{\xi_{j}}\right]_{u} \neq 0
$$

Então existem uma seqüência crescente $\left\{b_{i}: i \in \omega\right\} \in u$, e uma seqüência $\left\{E_{i}: i \in \omega\right\}$ de subconjuntos finitos de $\beta_{n}$ tais que:

(a) $\operatorname{supp}(x) \cup\left\{\xi_{j}: 0 \leq j \leq k_{f}\right\} \subseteq E_{0}$;

(b) $\omega \subseteq \bigcup_{i \in \omega} E_{i}$

(c) $E_{i+1} \supseteq \bigcup_{\xi \in E_{i}} \operatorname{supp}\left(f_{\xi}\left(b_{i}\right)\right) \bigcup E_{i}$;

(d) $\left\{f_{\xi}\left(b_{i}\right): \xi \in E_{i}\right\} \cup\left\{x_{\alpha}: \alpha \in E_{i}\right\}$ é independente, para todo $i<\omega$;

(e) $f\left(b_{i}\right)=\sum_{j=0}^{k_{f}} a_{j} f_{\xi_{j}}\left(b_{i}\right) \quad \forall i \in \omega$.

Demonstração: Definimos por induçào uma família $\left\{F_{k}: k \in \omega\right\}$ de subconjuntos finitos de $\beta_{n}$ :

$F_{0}=\operatorname{supp}(x) \bigcup\left\{\xi_{j}: 0 \leq j \leq h_{f}\right\}$,

$F_{k+1}=\bigcup_{\xi \in F_{k}}\left\{\operatorname{supp}\left(f_{\xi}(m)\right), m \leq k\right\} \cup F_{k} \cup k, \forall k \in \omega$.

Sejam, para cada $k \in \omega$

$A_{k}^{0}=\left\{m \in \omega:\left\{f_{\xi}(m): \xi \in F_{k} \cap I_{0}\right\} \cup\left\{x_{\alpha}: \alpha \in F_{k} \cap \beta_{d}\right\}\right.$ é independente $\}$, 
$A_{k}^{1}=\left\{m \in \omega:\left\{f_{\xi}(m): \xi \in F_{k} \cap I_{1}\right\} \cup\left\{x_{\alpha}: \alpha \in F_{k} \cap \beta_{n} \backslash \beta_{d}\right\}\right.$ é independente $\}$,

$U=\left\{m \in \omega: f(m)=\sum_{j=0}^{k_{j}} a_{j} f_{\xi_{j}}(m)\right\}$.

Como $\left\{\left[f_{\xi}\right]_{u}: \xi \in I_{0}\right\} \bigcup\left\{\left[\vec{x}_{\alpha}\right]_{u}: \alpha<\beta_{d}\right\}$ é independente e

$\left\{\left[f_{\xi}\right]_{u}: \xi \in I_{1}\right\} \bigcup\left\{\left[\vec{x}_{\alpha}\right]_{u}: \beta_{d} \leq \alpha<\beta_{n}\right\}$ é também independente temos que $A_{k}^{i} \in u$ para cada $i<2$. Portanto

$A_{k}=A_{k}^{0} \cap A_{k}^{1} \cap U=\left\{m \in \omega:\left\{f_{\xi}(m): \xi \in F_{k}\right\} \cup\left\{x_{\alpha}: \alpha \in F_{k}\right\}\right.$ é independente $\} \cap U \in$ $u, \forall k \in \omega$.

Como $u$ é $\mathrm{P}$-ponto, existe $A \in u$ tal que $A \backslash A_{k}$ é finito para todo $k<\omega$.

Seja $h: \omega \rightarrow \omega$ uma função crescente tal que $A \backslash A_{k} \subseteq h(k)$, para cada $k \in \omega$.

Pela seletividade de $u$, existe $B \in u$ que satisfaz

$$
B \cap h(1)=\emptyset, \quad B \subseteq A,|B \cap[h(k)+1, h(k+1)]| \leq 1 \forall k \in \omega .
$$

Seja $\left\{a_{k}: k \in \omega\right\}$ uma enumeraçào crescente de $B$. Entào $k<a_{k}<a_{m}$ e $a_{k} \in A_{k}$ para todo $k<m<\omega$. Definimos em $[\omega]^{2}$ uma coloração $P_{0}, P_{1}$ por:

$\{a, b\} \in P_{0}$ se e somente se $a, b \in B, a<b$ e $a_{k}<m$ se $a=a_{k}$ e $b=a_{m}$.

$\{a, b\} \in P_{1}$ caso contrário.

De novo pela seletividade de $u$, existe $C \in u, C \subseteq B$ tal que $[C]^{2} \subseteq P_{0}$ ou $[C]^{2} \subseteq P_{1}$. Seja $I \in{ }^{\omega} \omega$ tal que $C=\left\{a_{k}: k \in I\right\}$ e seja $\left\{i_{m}: m<\omega\right\}$ uma enumeração crescente de $I$. Suponhamos que $[C]^{2} \subseteq P_{1}$, então $a_{i_{0}} \geq i_{k}, \forall 1 \leq k<\omega$, o que é absurdo. Logo $[C]^{2} \subseteq P_{1}$ e portanto $i_{k}<a_{i_{k}}<i_{k+1}, \forall k<\omega$.

Definimos, para cada $k<\omega, b_{k}=a_{i_{k}}$ e $E_{k}=F_{i_{k}}$. Então

$$
\begin{aligned}
& E_{k} \cup \bigcup_{\xi \in E_{k}} \operatorname{supp}\left(f_{\xi}\left(b_{k}\right)\right)=F_{i_{k}} \cup \bigcup_{\xi \in F_{i_{k}}} \operatorname{supp}\left(f_{\xi}\left(a_{i_{k}}\right)\right) \subseteq \\
& \subseteq \quad F_{i_{k}} \cup \bigcup_{\xi \in F_{i_{k}}}\left\{\operatorname{supp}\left(f_{\xi}(m)\right), m<i_{k+1}\right\} \subseteq F_{i_{k+1}}=E_{k+1} .
\end{aligned}
$$


De $a_{i_{k}} \in A_{k}=A_{k}^{0} \cap A_{k}^{1} \operatorname{seguem}(\mathrm{d})$ e (f).

Lembramos que $d$ é divisor de $n$, e fixamos o inteiro $t$ que satisfaz $d . t=n$. Seja $f$ uma seqüencia em $G$ tal que

$$
[f]_{u}=\sum_{j=0}^{k_{f}} a_{j}\left[f_{\xi_{j}}\right]_{u},
$$

onde $a_{j} \in \mathbb{Z}$ e $\sum_{j=0}^{k_{f}} a_{j}\left[f_{\xi_{j}}\right]_{u} \neq 0$. Então existem $A \in u$ e um divisor $l_{f}$ de $n$ tais que $\operatorname{ord}(f(m))=l_{f} \forall m \in A$. Seja $l_{f}^{\prime} \in \mathbb{Z}$ tal que $l_{f} \cdot l_{f}^{\prime}=n$.

Lema 2.10. Seja $x \in G$ e seja $f$ uma seqüência $e m G$ tal que

$$
[f]_{u}=\sum_{j=0}^{k_{f}} a_{j}\left[f_{\xi_{j}}\right]_{u},
$$

onde $a_{j} \in \mathbb{Z}$ e $\sum_{j=0}^{k_{f}} a_{j}\left[f_{\xi_{j}}\right]_{u} \neq 0$. Se existe u um ultrafiltro seletivo sobre $\omega$, entäo existe um homomorfismo $\oint_{f, x}: G \rightarrow \mathbb{Z}(n)$ que satisfaz:

1) $\phi_{f, x}(x) \neq 0$ se $x \neq 0$,

2) $\left\{m \in \omega: \phi_{f, x}\left(f_{\xi}(m)\right)=l . \phi_{f, x}\left(x_{\xi}\right)\right\} \in u \quad \forall \xi \in I_{0}$,

3) $\left\{m \in \omega: \phi_{f, x}\left(f_{\xi}(m)\right)=\phi_{f, x}\left(x_{\xi}\right)\right\} \in u, \forall \xi \in I_{1}$,

4) $\left\{m \in \omega: \phi_{f, x}(f(m)) \neq \sum_{j=0}^{k_{f}} t_{\xi_{j}} \cdot a_{j} \phi_{f, x}\left(x_{\xi_{j}}\right)\right\}$ é infinito, onde $t_{\xi_{j}}=t$ se $\xi_{j} \in I_{0} \epsilon$ $t_{\xi}=1$ se $\xi_{j} \in I_{1}$.

Demonstração: Pelo Lema 2.9 existem uma seqüência crescente $\left\{b_{i}: i \in \omega\right\} \in u$ e uma seqüência $\left\{E_{i}: i \in \omega\right\}$ de subconjuntos finitos de $\beta_{n}$ tais que:

(a) $\operatorname{supp}(x) \cup\left\{\xi_{j}: 0 \leq j \leq k_{f}\right\} \subseteq E_{0}$;

(b) $\omega \subseteq \bigcup_{i \in \omega} E_{i}$; 
(c) $E_{i+1} \supseteq \bigcup_{\xi \in E_{i}} \operatorname{supp}\left(f_{\xi}\left(b_{i}\right)\right) \bigcup E_{i}$;

(d) $\left\{f_{\xi}\left(b_{i}\right): \xi \in E_{i}\right\} \cup\left\{x_{\alpha}: \alpha \in E_{i}\right\}$ é independente, para todo $i<\omega$;

(e) $f\left(b_{i}\right)=\sum_{j=0}^{k_{f}} a_{j} f_{\xi_{j}}\left(b_{i}\right) \quad \forall i \in \omega$.

Como $\left\{b_{i}: i \in \omega\right\} \in u$ temos que $\left\{b_{2 i}: i \in \omega\right\} \in u$ ou $\left\{b_{2 i+1}: i \in \omega\right\} \in u$. Podemos assumir s.p.g. que $\left\{b_{2 i}: i \in \omega\right\} \in u$.

Também podemos assumir que ord $\left(f\left(b_{i}\right)\right)=l_{f}$ para todo $i \in \omega$.

Definimos primeiro $\phi_{f, x}$ em $\left\{x_{\alpha}: \alpha \in \bigcup_{i \in \omega} E_{i}\right\}$ por indução.

Para cada $\alpha \in E_{0}$, definimos $\phi_{f, x}\left(x_{x}\right)$ de tal forma que $\phi_{f, x}(x) \neq 0$.

Suponhamos que para $h \in \omega$, temos definida $\phi_{f, x}$ em $\left\{x_{\alpha}: \alpha \in E_{h}\right\}$, tal que para cada $k<h$,

i) se $k$ é par, então

$\phi_{f, x}\left(f_{\xi}\left(b_{k}\right)\right)=t . \phi_{f, x}\left(x_{\xi}\right) \quad \forall \xi \in E_{k} \cap I_{0} \quad$ e $\phi_{f, x}\left(f_{\xi}\left(b_{k}\right)\right)=\phi_{f, x}\left(x_{\xi}\right) \quad \forall \xi \in E_{k} \cap I_{1} ;$

ii) se $k$ é impar, então

$\phi_{f, x}\left(f\left(b_{k}\right)\right)=\sum_{j=0}^{k_{f}} t_{\xi_{j}} \cdot a_{j} \phi_{f, x}\left(x_{\xi_{j}}\right)+l_{f}^{\prime}$, onde $t_{\xi_{j}}=t$ se $\xi_{j} \in I_{0}$ e $t_{\xi_{j}}=1$ se $\xi_{j} \in I_{1}$.

Observamos que $\phi_{f, x}\left(f\left(b_{k}\right)\right)$ assim definida satisfaz ord $\left(\phi_{f, x}\left(f\left(b_{k}\right)\right)\right)$ divide $l_{f}=\operatorname{ord}\left(f\left(b_{k}\right)\right)$.

Como $\left\{f_{\xi}\left(b_{h}\right): \xi \in E_{h}\right\} \cup\left\{\left\{x_{\alpha}\right\}: \alpha \in E_{h}\right\}$ é independente, podemos estender $\phi_{f, \alpha}$ a $\left\{x_{\alpha}: \alpha \in E_{h+1}\right\}$ satisfazendo:

i) se $h$ é par:

$\phi_{f, x}\left(f_{\xi}\left(b_{h}\right)\right)=\phi_{f, x}\left(t . x_{\xi}\right) \quad \forall \xi \in E_{h} \cap I_{0}$ e $\phi_{f, x}\left(f_{\xi}\left(b_{h}\right)\right)=\phi_{f, x}\left(x_{\xi}\right) \quad \forall \xi \in E_{h} \cap I_{1} ;$

ii) se $h$ é impar:

$\phi_{f, x}\left(f\left(b_{h}\right)\right)=\sum_{j=0}^{k_{f}} t_{\xi_{j}} \cdot a_{j} \phi_{f, x}\left(x_{\xi_{j}}\right)+l_{f}^{\prime}$, onde $t_{\xi_{j}}=t$ se $\xi_{j} \in I_{0}$ e $t_{\xi_{j}}=1$ se $\xi_{j} \in I_{1}$. 
Temos então definido o homomorfismo $\phi_{f, x}$ no grupo gerado por $\left\{x_{\alpha}: \alpha \in \bigcup_{i \in \omega} E_{i}\right\}$ e verifica:

$$
\begin{aligned}
& \left\{m \in \omega: \phi_{f, x}\left(f_{\xi}(m)\right)=\phi_{f, x}\left(t . x_{\xi}\right)\right\} \in u \forall \xi \in\left(I_{0} \cap \bigcup_{i \in \omega} E_{i}\right), \\
& \left\{m \in \omega: \phi_{f, x}\left(f_{\xi}(m)\right)=\phi_{f, x}\left(x_{\xi}\right)\right\} \in u, \forall \xi \in\left(I_{1} \cap \bigcup_{i \in \omega} E_{i}\right), \\
& \left\{m \in \omega: \phi_{f, x}(f(m))=\sum_{j=0}^{k_{j}} t_{\xi_{j}} \cdot a_{j} \phi_{f, x}\left(x_{\xi_{j}}\right)+l_{f}^{\prime}\right\} \text { é infinito, onde } t_{\xi_{j}}=t \text { se } \xi_{j} \in I_{0}
\end{aligned}
$$
e $t_{\xi_{j}}=1$ se $\xi_{j} \in I_{1}$.

Para cada $\alpha \in \beta_{d} \backslash \bigcup_{i \in \omega} E_{i}$, podemos por $\phi_{f, x}\left(x_{\alpha}\right)=0$.

Resta então definirmos $\phi_{f, x}\left(x_{\alpha}\right)$ para cada $\alpha \in \beta_{n} \backslash\left(\beta_{d} \cup \bigcup_{i \in \omega} E_{i}\right)$. Esta definição vai ser feita por indução transfinita. Seja $\gamma \in \beta_{n} \backslash\left(\beta_{d} \cup \bigcup_{i \in \omega} E_{i}\right)$ e suponhamos $\phi_{f, x}$ definida em $\left\{x_{\alpha}: \alpha \in \beta_{d} \cup \bigcup_{i \in \omega} E_{i} \cup \gamma\right\}$. Sabemos que $\bigcup_{k \in \omega} \operatorname{supp}\left(f_{\gamma}(k)\right) \subseteq \gamma$, portanto podemos definir $\phi_{f, x}$ tal que:

$\phi_{f, x}\left(t x_{\gamma}\right)=u-\lim _{k \in \omega}\left(\phi_{f, x}\left(f_{\gamma}(k)\right)\right.$ se $\gamma \in I_{0}$,

$\phi_{f, x}\left(x_{\gamma}\right)=u-\lim _{k \in \omega^{\prime}}\left(\phi_{f, x}\left(f_{\gamma}(k)\right)\right.$ se $\gamma \in I_{1}$.

Demonstração do Teorema 2.8: Para cada $x \in G$ e para cada seqüencia $f$ injetora de $G \operatorname{com}[f]_{u}=\sum_{j=0}^{k_{f}} a_{j} \cdot\left[f_{\xi_{j}}\right]_{u}$, onde $a_{j} \in \mathbb{Z}$ e $\sum_{j=0}^{k_{f}} a_{j} \cdot\left[f_{\xi_{j}}\right]_{u} \neq 0$, seja $\phi_{f, x}$ como no Lema 2.10.

Definimos o homomorfismo $\varphi: G \rightarrow \mathbb{Z}(n)^{\beta_{n}}$ por

$$
\varphi(z)=\triangle_{(f, x)} \phi_{f, x}(z)
$$

Vemos que se $x \in G \operatorname{com} x \neq 0$, entào $\varphi(x) \neq 0$ pois $\phi_{f, x}(x) \neq 0$. Portanto o homomorfismo $\varphi$ é injetor e $\varphi(G)$ é um subgrupo de $\mathbb{Z}(n)^{\beta_{n}}$ isomorfo a $G$. 
Vamos verificar agora que $\varphi(G)$ com a topologia de subespaço de $\mathbb{Z}(n)^{\beta_{n}}$ é enumeravelmente compacto.

Seja $\left(z_{m}\right)_{m<w}$ uma seqüência qualquer em $\varphi(G)$. Então existe $g \in G^{w}$ tal que $z_{m}=\varphi(g(m)), \forall m \in \omega$. Se $[g]_{u}=\sum_{i=0}^{k_{g}} a_{i} \cdot\left[f_{\xi_{i}}\right]_{u}+\sum_{l=0}^{h_{g}} c_{l} \cdot\left[x_{\alpha_{l}}\right]_{u}$, seja.

$$
A=\left\{m \in \omega: g(m)=\sum_{i=0}^{k_{g}} a_{i} \cdot f_{\xi_{i}}(m)+\sum_{l=0}^{h_{g}} c_{l}, x_{\alpha_{l}}\right\} .
$$

Como $A \in u$, do Lema 2.10, segue que para cada par $(f, x)$,

$$
u-\lim _{m \in \omega} \phi_{f, x}(g(m))=\sum_{i=0}^{k_{g}} t_{\xi_{i}} \cdot a_{i} \cdot \phi_{f, x}\left(x_{\xi_{i}}\right)+\sum_{l=0}^{h_{g}} c_{l} \cdot \phi_{f, x}\left(x_{\alpha_{l}}\right),
$$

onde $t_{\xi_{i}}=t$ se $\xi_{i} \in I_{0}$ e $t_{\xi_{i}}=1$ se $\xi_{i} \in I_{1}$.

Logo,

$$
u-\lim _{m \in \omega} z_{m i}=u-\lim _{m \in \omega} \varphi(g(m))=\sum_{i=0}^{k_{g}} t_{\xi_{i}} \cdot a_{i} \cdot \varphi\left(x_{\xi_{i}}\right)+\sum_{l=0}^{h_{g}} c_{l} \cdot \varphi\left(x_{\alpha_{l}}\right),
$$

onde $t_{\xi_{i}}=t$ se $\xi_{i} \in I_{0}$ e $t_{\xi_{i}}=1$ se $\xi_{i} \in I_{1}$.

Só falta verificar que $\varphi(G)$ nào possui seqüências não triviais convergentes.

Seja $\{\varphi(f(m)): m \in \omega\}$ uma seqüência não trivial. Vamos assumir que a sequência $\{\varphi(f(m)): m \in \omega\}$ é injetiva. Como $\varphi$ é injetiva, temos que $f \in G^{\omega}$ também é injetiva e que $[f]_{u}=\sum_{j=0}^{k_{f}} a_{j} \cdot\left[f_{\xi_{j}}\right]_{u}$, onde $a_{j} \in \mathbb{Z}$ e $\sum_{j=0}^{k_{f}} a_{j} \cdot\left[f_{\xi_{j}}\right]_{u} \neq 0$. Também assumimos que existem $A \in u$ e $l_{f}$ divisor de $n$ tais que ord $(f(m))=l_{f} \forall m \in \omega$. Segue do item 4) do Lema 2.10 que $\left\{m \in \omega: \phi_{f, x}(f(m))=\sum_{j=0}^{k_{f}} t_{\xi_{j}} \cdot a_{j} \phi_{f, x}\left(x_{\xi_{j}}\right)+l_{f}^{\prime}\right\}$ é infinito, onde $l_{f}^{\prime}$ é tal que $l_{f}, l_{f}^{\prime}=n$. Mas

$$
u-\lim _{m \in \omega} \varphi(f(m))=u-\lim _{m \in \omega} \phi_{f, x}(f(m))=\sum_{j=0}^{k_{f}} t_{\xi_{j}} \cdot a_{j} \cdot \phi_{f, x}\left(x_{\xi_{j}}\right) .
$$

Portanto a seqüência $\{\varphi(f(m)): m \in \omega\}$ não converge. 
Corolário 2.11. Se existe um ultrafiltro seletivo u sobre $\omega$, então para um grupo abeliano de torção $G$, de cardinalidade $\mathfrak{c}$ são equivalentes:

(a) G tem ordem limitada n e para cada divisor próprio d de n, o subgrupo dG é finito ou tem cardinalidade $\mathfrak{c}$.

(b) G tem ordem limitada $n$, e para cada componente prima $G_{r}$ de $G$ o último invariante infinito de Ulm-Kaplansky de $G_{r}$ éc.

(c) G admite uma topologia de grupo topológico u-compacta sem seqüências não triviais convergentes.

Demonstração: A equivalência entre (a) e (b) é o Lema 1.6.

Que (b) implica (c) é conseqüência imediata do Teorema 2.8 e é o único passo da equivalência que precisa da existência de um ultrafiltro seletivo.

Para $(\mathrm{c}) \Rightarrow(\mathrm{a})$, lembramos que um espaço topológico $u$-compacto é pseudocompacto. Comfort e van Mill provaram em [7] que um grupo abeliano de torção pseudocompacto de cardinalidade c verifica (a). Vamos aqui fazer um esboço da demonstração que aparece em [8]. Vamos primeiro assumir que $G$ têm ordem $n$, e vamos provar que $d G$ é finito ou tem cardinalidade c. O homomorfismo $\pi: G \rightarrow d G$ que leva $x \in G$ em $d . x \in d G$ é sobrejetor e contínuo. Como a imagem contínua de um espaço pseudocompacto é também um espaço pseudocompacto, temos que o grupo $d G$ é pseudocompacto. Um grupo pseudocompacto é finito e discreto ou tem cardinalidade maior ou igual a c . Portanto $d G$ é finito ou $|G|=\mathfrak{c}$.

A prova de que um grupo abeliano de torção pseudocompacto é de ordem limitada, não é tão simples. O que segue é só um esboço da demonstração.

A idéia é mostrar que todo subgrupo enumerável de $G$ tem ordem limitada. Se isso acontece, então $G$ também tem ordem limitada. 
Fixamos um subgrupo enumerável qualquer $G^{\prime}$ de $G$. O grupo $G$, sendo pseudocompacto, é totalmente limitado. Sendo assim, por ser $G^{\prime}$ enumerável é possivel construir um homomorfismo contínuo $\pi$ de $G$ sobre um grupo métrico $H$, tal que a restriçào de $\pi$ a $G^{\prime}$ é injetora.

Seja $K=\operatorname{ker} \pi$ e seja $\beta: G \rightarrow G / K$ o homomorfismo natural. Entào existe um isomorfismo contínuo $i: G / K \rightarrow H$ com $\pi=i \circ \beta$. Observamos que o grupo $G / K$ é pseudocompacto por ser imagem contínua de $G$ e também é um grupo de torção. Mas é possível provar que existe uma uniformidade completa e totalmente limitada sobre $G / K$ que induz a topologia de $G / K$. Portanto o grupo $G / K$ é compacto. Da classificação dos grupos topológicos compactos ( Teorema 1.38), segue que o grupo $G / K$ tem ordem limitada.

$\beta\left(G^{\prime}\right)$ é subgrupo de $G / K$, e portanto tem ordem limitada também. Mas o homomorfismo $\beta$ restrito a $G^{\prime}$ é um isomorfismo do qual segue que $G^{\prime}$ tem ordem limitarla.

Corolário 2.12. (GCH) Seja G um grupo abeliano de torçào e u um ultrafiltro seletivo sobre w. Então são equivalentes:

(a) G admite uma topologia de grupo u-compacta sem seqüencias não triviais convergentes;

(b) G admite uma topologia de grupo enumeravelmente compacta sem seqüências não triviais convergentes;

(c) G admite uma topologia de grupo pseudocompacta;

(d) para cada número primo $r$, a componente prima $G_{r}$ de $G$ admite uma topologia de grupo pseudocompacta e $G_{r}$ é não trivial só para uma quantidade finita de $r$ 's:

(e) para cada componente primária $G_{r}$ de $G$ e cada invariante de Ulm-Kaplansky infinita $\alpha_{r k}$ de $G_{r}$, correspondente a $\mathbb{Z}\left(r^{k}\right)$, existe um inteiro $k^{\prime} \geq k$ tal que a invariante 
$\alpha_{r \cdot k^{\prime}} \quad$ satisfaz $\alpha_{r k} \leq \alpha_{r^{\prime}} \quad$ e $\left(\alpha_{r k^{\prime}}\right)^{\omega}=\alpha_{r k^{\prime}}$

Demonstração: (a) $\Rightarrow(\mathrm{b}) \Rightarrow(\mathrm{c}) \Rightarrow(\mathrm{d})$ são resultados conhecidos.

$(\mathrm{e}) \Rightarrow(\mathrm{a})$ é o Teorema 2.8. Vamos ver por que vale $(\mathrm{d}) \Rightarrow(\mathrm{e})$. Para isso vamos definir cardinal de van Douwen: dizemos que um cardinal $\kappa$ é um cardinal de van Douwen se $\kappa$ é tal que $\kappa \geq \mathfrak{c}$ e se $\kappa$ é limite forte, então $\operatorname{cf}(\kappa)>\omega$.

Esta defiinição é natural se levamos en conta a restrição dada por van Douwen à cardinalidade de um grupo topológico pseudocompacto infinito.

Em [8], Teorema 1.5, os autores mostraram que a condiçào (d) implica que para cada componente primária $G_{r}$ de $G$ e cada invariante de Ulm-Kaplansky infinita $\alpha_{r k}$ de $G_{r}$, correspondente a $\mathbb{Z}\left(r^{k}\right)$, existe um inteiro $k^{\prime} \geq k$ tal que a invariante $\alpha_{r^{\prime} k^{\prime}}$ satisfaz $\alpha_{r^{k}} \leq \alpha_{r^{k^{\prime}}}$ e $\alpha_{r^{k^{\prime}}}$ é um cardinal de van Douwen. Mas sob GCH, se $\kappa$ é um cardinal de van Douwen, então $\kappa^{\omega}=\kappa$. Portanto temos (e). 


\section{Capítulo 3}

\section{Ultrafiltros seletivos e grupos de torção enumeravelmente}

\section{compactos.}

Neste capítulo apresentamos uma condiçào suficiente para que, sob a existência de $\mathfrak{c}$ ultrafiltros seletivos, um grupo abeliano de torção $G$, de cardinalidade $\mathfrak{c}$, admita, para cada número natural $p$, uma topologia $\tau$ de grupo topológico tal que $(G, \tau)^{p}$ é enumeravelmente compacto, mas $(G, \tau)^{q}$ não é enumeravelmente compacto para algum $q>p$. 


\subsection{Uma topologia enumeravelmente compacta para}

$$
\mathbb{Z}(r)^{(\mathfrak{c})} \text {. }
$$

Começamos apresentando a construção de uma topologia enumeravelmente compacta para $\mathbb{Z}(r)^{(\mathfrak{c})}$, onde $r$ é um número primo. Acreditamos que a leitura deste caso pode ajudar na compreensão do caso mais geral.

Teorema 3.1. Seja $G=\mathbb{Z}(r)^{(\mathfrak{c})}$, e p um inteiro positivo. Se existem $\mathfrak{c}$ ultrafiltros seletivos incomparáveis sobre $\omega$, então existe uma topologia $\tau$ de grupo topológico tal que $(G, \tau)^{p}$ é enumeravelmente compacto, mas $(G, \tau)^{p+1}$ não é enumeravelmente compacto.

A idéia é, como no capítulo anterior, construir um monomorfismo $\varphi: G \rightarrow \mathbb{Z}(r)^{\mathrm{i}}$ tal que $\varphi(G)$ com a topologia de subespaço de $\mathbb{Z}(r)^{c}$, possua as propriedades requeridas. Também como no caso do capítulo anterior, para fazer de $\varphi$ uma funçào injetora, trabalhamos com um elemento do grupo de cada vez, construindo homomorfismos $\phi_{x}: G \rightarrow \mathbb{Z}(r)$

Seja $\left\{x_{\alpha, i}: \alpha<\mathfrak{c}, i<p+1\right\}$ uma base para o espaço vetorial $\mathbb{Z}(r)^{(\mathfrak{c})}$.

Enumeramos por $\left\{f_{\xi}: \omega \leq \xi<c\right\}$ as funçòes $f: S \times \omega \rightarrow G$ com $S \in(p+1) \leq p$ tais que para cada $F \in] \mathfrak{c} \times(p+1)[<\omega$ o conjunto

$\omega \backslash\left\{m \in \omega:\left\{x_{\alpha, i}:(\alpha, i) \in F\right\} \cup\{f(i, m): i \in S\}\right.$ é linearmente independente $\}$ é finito.

Para cada $\xi$, chamamos de $S_{\xi}$ o subconjunto de $(p+1)$ tal que $\operatorname{dom}\left(f_{\xi}\right)=S_{\xi} \times \omega$. Assumimos que $\bigcup_{(i, m) \in d o m\left(f_{\xi}\right)} \operatorname{supp}\left(f_{\xi}(i, m)\right) \subseteq \xi \times(p+1)$. 
Lema 3.2. Seja $\varphi: G \rightarrow \mathbb{Z}(r)^{\mathfrak{e}}$ um monomorfismo de grupos e seja $\left\{u_{\xi}: \omega \leq\right.$ $\xi<\mathfrak{c}\}$ uma família de ultrafiltros sobre $\omega$. Se para cada $\omega \leq \xi<\mathfrak{c}$, a seqüencia $\left\{\left(\varphi\left(f_{\xi}(i, m)\right)\right)_{i \in S_{\xi}}: m \in \omega\right\}$ tem $u_{\xi}$-limite $\left(\varphi\left(x_{\xi, i}\right)\right)_{i \in S_{\xi}} \in m \varphi(G)^{s_{\xi}}$, então $\varphi(G)^{p} \dot{\epsilon}$ enumeravelmente compacto como subespaço de $\mathbb{Z}(r)^{c}$.

Demonstração: Seja $\left\{\left(\varphi\left(a_{i, m}\right)\right)_{i<p}: m \in \omega\right\}$ uma seqüência em $\varphi(G)^{p}$.

Afirmação 1: Existem $S \subseteq p, c_{i} \in G$ e $E_{i} \subseteq S$ para cada $i<p, A \subseteq \omega$ infinito e subconjuntos $\left\{b_{i, m}: m \in \omega\right\}$ de $G$ para cada $i \in S$, tais que:

$\left\{\left(\varphi\left(b_{i, m}\right)\right)_{i \in S}: m \in A\right\}$ tem ponto de acumulação em $\varphi(G)^{S} \mathrm{e}$

$c_{i}=a_{i, m}-\sum_{j \in E_{i}} b_{j, m}$ para cada $m \in A$.

Antes de demonstrarmos a Afirmação 1, vamos fazer uso dela para demonstrar a Afirmaçào g: A seqüência $\left\{\left(\varphi\left(a_{i, m}\right)\right)_{i<p}: m \in \omega\right\}$ possui ponto de acumulaçào em $\varphi(G)^{p}$

Demonstração da Afirmaçào $2:$ Sejam $S \subseteq p, c_{i} \in G$ e $E_{i} \subseteq S$ para cada $i<p, A \subseteq w$ infinito e subconjuntos $\left\{b_{i, m}: m \in \omega\right\}$ de $G$ para cada $i \in S$, como na Afirmação 1 . Entào existe um ultrafiltro $u$ sobre $\omega$ e $b_{i} \in G$ para cada $i \in S$, tais que

$$
\varphi\left(b_{i}\right)=u-\lim _{m \in \omega} \varphi\left(b_{i, m}\right) \text { para cada } i \in S
$$

Logo, para cada $i<p$ temos que

$$
\begin{aligned}
& u-\lim _{m \in \omega} \varphi\left(a_{i, m}\right)=\varphi\left(c_{i}\right)+u-\lim _{m \in \omega} \sum_{j \in E_{i}} \varphi\left(b_{j, m}\right)= \\
& =\varphi\left(c_{i}\right)+\sum_{j \in E_{i}}\left(u-\lim _{m \in \omega} \varphi\left(b_{j, m}\right)\right)=\varphi\left(c_{i}\right)+\sum_{j \in E_{i}} \varphi\left(b_{j}\right) .
\end{aligned}
$$

Portanto cada sequência $\left\{\varphi\left(a_{i, m}\right): m \in \omega\right\}$ tem $u$ - limite em $\varphi(G)$. Segue que $\left\{\left(\varphi\left(a_{i, m}\right)_{i<p}: m \in \omega\right\}\right.$ tem $u$-limite em $\varphi(G)^{p}$. 
Demonstração da Afirmação 1: Seja u um ultrafiltro seletivo sobre $\omega$. Consideramos o ultraproduto ${ }^{\omega} G / u$, que é um espaço vetorial sobre $\mathbb{Z}(r)$. Chamamos $R$ ao subespaço de ${ }^{\omega} G / u$ gerado por $\left\{\left[\left\{a_{i, m}: m \in \omega\right\}\right]_{u}: i<p\right\}$. Isto é:

$$
R=\left\langle\left\{\left[\left\{a_{i, m}: m \in \omega\right\}\right]_{u}: i<p\right\}\right\rangle
$$

Podemos considerar $C \subseteq G$ tal que $\left\{[\vec{x}]_{u}: x \in C\right\}$ é uma base para $R \cap\left\{[\vec{x}]_{u}: x \in G\right\}$. Sejam $S \subseteq p$ e $\left\{\left[\left\{b_{i, m}: m \in \omega\right\}\right]_{u}: i \in S\right\}$ tais que $\left\{\left[\left\{b_{i, m}: m \in \omega\right\}\right]_{u}: i \in S\right\} \bigcup\left\{[\vec{x}]_{u}: x \in C\right\}$ é uma base para $R$.

Escrevemos cada elemento do conjunto gerador original de $R$ como combinação linear dos elementos da base:

para cada $i<p$, sejam $E_{i} \subseteq S$ e $C_{i} \subseteq C$ tais que

$$
\left[\left\{a_{i, m}: m \in \omega\right\}\right]_{u}=\sum_{x \in C_{i}} c_{i, x}[\vec{x}]_{u}+\sum_{j \in E_{i}} t_{j, i}\left[\left\{b_{j, m}: m \in \omega\right\}\right]_{u},
$$

onde $c_{i, x} \in \mathbb{Z}(r) \forall x \in C_{i}$ e $t_{j, i} \in \mathbb{Z}(r) \forall j \in E_{i}$.

Segue que a seqüência $\left\{a_{i, m}: m \in \omega\right\}-\sum_{j \in E_{i}}\left\{t_{j, i} \cdot b_{j, m}: m \in \omega\right\}$ é u-equivalente a uma sequência constante. Portanto existe $B \in u$ e elementos $x_{i}$ do grupo $G$ tais que para cada $i<p$

$$
x_{i}=\sum_{x \in C_{i}} c_{i, x}, x=a_{i, m}-\sum_{j \in E_{i}} t_{j, i} \cdot b_{j, m} \quad \forall m \in B
$$

(*) Para cada $T \subseteq S$ não vazio, e para cada conjunto $\left\{t_{i}: i \in T\right\} \subseteq \mathbb{Z}(r)$, se $\sum_{i \in T} t_{i}\left[\left\{b_{i, m}: m \in \omega\right\}\right]_{u} \neq 0$ então $\sum_{i \in T} t_{i}\left[\left\{b_{i, m}: m \in \omega\right\}\right]_{u}$ nào está na classe de $u^{-}$ equivalência de uma seqüência constante. Isto é verdade pois

$$
\sum_{i \in T} t_{i}\left[\left\{b_{i, m}: m \in \omega\right\}\right]_{u} \in R
$$


e é uma combinação linear de elementos da base de $R$ que não geram as constantes. Agora, para cada $T \subseteq S$ e para cada $\left\{t_{i}: i \in T\right\}$ que abreviamos por $\left\{t_{i}\right\}$ definimos a. aplicação $h_{T,\left\{t_{i}\right\}}: \omega \rightarrow G$ por

$$
h_{T,\left\{t_{i}\right\}}(m)=\sum_{i \in T} t_{i} \cdot b_{i, m}
$$

Se $\sum_{i \in T} t_{i}\left[\left\{b_{i, m}: m \in \omega\right\}\right]_{u} \neq 0$, por $(*)$ e por ser $u$ seletivo podemos afirmar que existe $A_{T,\left\{t_{i}\right\}} \in u, A_{T,\left\{t_{i}\right\}} \subseteq B$, tal que a aplicação $h_{T,\left\{t_{i}\right\}} \mid A_{T,\left\{t_{i}\right\}}$ é injetora. Como só temos uma quantidade finita de funçòes $h_{T,\left\{t_{i}\right\}}$,

(**) existe $A \in u, A \subseteq B$ tal que $h_{T,\left\{t_{i}\right\}} i_{A}$ é injetora, para cada $T \subseteq S$ e para cada $\left\{t_{i}: i \in T\right\}$.

Seja $\left\{m_{k}: k \in \omega\right\}$ uma enumeraçào de $A$ e seja a funçào $f: S \times \omega \rightarrow G$ definida por $f(i, k)=b_{i, m_{k}}, \forall i \in S, \forall k \in \omega$.

Afirmação: existe $\omega \leq \xi<\mathfrak{c}$, tal que $f_{\xi}=f$.

Para demonstrar esta afirmaçào, precisamos provar que para cada subconjunto finito $F$ de $\mathfrak{c} \times(p+1)$, o conjunto

$X_{F}=\omega \backslash\left\{m \in \omega:\left\{x_{\alpha, i}:(\alpha, i) \in F\right\} \cup\{f(i, m): i \in S\}\right.$ é linearmente independente $\}$

é finito. Seja entào $F$ um subconjunto finito de $\mathfrak{c} \times(p+1)$, e vamos supor, por absurdo, que o conjunto $X_{F}$ é infinito. Então, para cada $k \in X_{F}$, existe $F_{k} \subseteq F$, $\emptyset \neq T_{k} \subseteq S$ e inteiros $t_{i}^{k}$ para cada $i \in T_{k}$ tais que $\sum_{i \in T_{k}} t_{i}^{k} \cdot b_{i, m_{k}}$ é combinação linear dos elementos do conjunto $\left\{x_{\alpha}: \alpha \in F_{k}\right\}$. Como $F$ é finito, $S$ também é finito e os escalares que aparecem nas combinações lineares são elementos de $\mathbb{Z}(r)$ que também é finito, temos que existe um subconjunto infinito $Y_{F}$ de $X_{F}, E \subseteq F, T \subseteq S$ e inteiros 
$\left\{t_{i}: i \in T\right\}$ tais que $F_{k}=E, T_{k}=T,\left\{t_{i}^{k}: i \in T_{k}\right\}=\left\{t_{i}: i \in T\right\}$ para todo $k \in Y_{F}$ e $\left\{\sum_{i \in T} t_{i}, b_{i, m_{k}}: k \in Y_{F}\right\}$ é unitário. Mas isto contradiz $(* *)$. Portanto a suposição é falsa e temos demonstrada a afirmação.

Por hipótese temos que $\left\{\varphi\left(f_{\xi}(i, k)\right)_{i \in S_{\xi}}: k \in \omega\right\}$ tem $u_{\xi}$ limite $\left(\varphi\left(x_{\xi, i}\right)\right)_{i \in S_{\xi}}$ em $\varphi(G)^{S_{\xi}}$. Segue que $\left\{\left(\varphi\left(b_{i, m}\right)\right)_{i \in S}: m \in A\right\}$ possui ponto de acumulação em $(\varphi(G))^{S}$ e que $c_{i}=a_{i, m}-\sum_{j \in E_{i}} b_{j, m}$ para cada $m \in A$.

Vimos com este lema que só temos que nos preocupar com os pontos de acumulação das seqüências $f_{\xi}$.

O seguinte lema de A.H. Tomita é fundamental para esta construção.

Lema 3.3. [32] Sejam $\left\{u_{j}: j \in \omega\right\}$ conjunto de ultrafiltros seletivos incomparáveis sobre $\omega$, e para cada $j \in \omega\left\{a_{k}^{j}: k \in \omega\right\} \in u_{j}$ uma seqüencia estritamente crescente tal que $k<a_{h}^{j}, \forall k \in \omega$. Então para cada $j \in \omega$ existe um conjunto $I_{j} \subseteq \omega$ tal que:

i) $\left\{a_{k}^{j}: k \in I_{j}\right\} \in u_{j}, \forall j<\omega e$

ii) os clementos do conjunto $\left\{\left[k, a_{k}^{j}\right]: k \in \bigcup_{j<\omega} I_{j}\right\}$ são dois a dois disjuntos.

Lema 3.4. Sejam $H$ um subconjunto infinito enumerável de $(\mathfrak{c} \backslash \omega), \quad\left\{u_{\xi}: \xi \in H\right\}$ conjunto de ultrafiltros seletivos incomparáveis de $\omega$ e F um subconjunto finito de $\mathfrak{c} \times(p+1)$.

Seja E um subconjunto enumerável de $\mathfrak{c} \times(p+1)$ tal que $F \cup(\omega \times(p+1)) \subseteq E$, $H \times(p+1) \subseteq E \quad e \quad \bigcup_{(k, m) \in S_{\xi} \times \omega} \operatorname{supp}\left(f_{\xi}(k, m)\right) \subseteq E$, para cada $\xi \in H$.

Entâo existe uma seqüência crescente $\left\{b_{k}: k \in \omega\right\} \subseteq \omega$, uma função $r: \omega \rightarrow H e$ uma seqüência $\left\{E_{k}: k \in \omega\right\}$ de subconjuntos finitos de $E$ tais que: 
(a) $F \subseteq E_{0}$;

(b) $E=\bigcup_{k \in \omega} E_{k}$;

(c) $E_{k+1} \supseteq\left\{\bigcup_{i \in S_{\xi}} \operatorname{supp}\left(f_{\xi}\left(i, b_{k}\right)\right):\{\xi\} \times(p+1) \subseteq E_{k}\right\} \cup E_{k}$;

(d) $\left\{f_{r(k)}\left(i, b_{k}\right): i \in S_{r(k)}\right\} \cup\left\{x_{\alpha, i}:(\alpha, i) \in E_{k}\right\}$ é linearmente independente, para cada $k \in \omega:$

(e) $\left\{b_{k}: k \in r^{-1}(\{\xi\})\right\} \in u_{\xi}$, para cada $\xi \in H$;

(f) $E_{k} \cap(\omega \times(p+1))=N_{k} \times(p+1)$, para algum $N_{k} \in \omega$.

Demonstração: Definimos por indução uma seqüência $\left\{F_{k}: k \in \omega\right\}$ de subconjuntos finitos de $E$, como segue:

1) $F_{0}=F$;

2) $F_{k+1} \in[E]^{<\omega}$ tal que $F_{k+1} \supseteq F_{k} \cup \bigcup_{m \leq k}\left\{\bigcup_{i \in S_{\xi}} \operatorname{supp}\left(f_{\xi}(i, m)\right):\{\xi\} \times S_{\xi} \subseteq F_{k}\right\}$, e se $m \in \omega$ e $(m, i) \in F_{k+1}$ para algum $i<p+1$, então $(m+1) \times(p+1) \subseteq F_{k+1}$;

3) $E=\bigcup_{k \in \omega} F_{k}$.

Para cada $\xi \in H$ e para cada $k \in \omega$, seja $A_{k}^{\xi}=\left\{m \in \omega:\left\{f_{\xi}(i, m): i \in S_{\xi}\right\} \cup\left\{x_{\alpha, i}:(\alpha, i) \in F_{k}\right\}\right.$ é l.i. $\}$

Pela definição de $f_{\xi}$, temos que $A_{k}^{\xi} \in q_{\xi}, \forall k \in \omega, \forall \xi \in H$.

Como $u_{\xi}$ é ultrafiltro seletivo, é também $P$-ponto. Logo existe $A_{\xi} \in u_{\xi}$ que satisfaz 4) $A_{\xi} \backslash A_{k}^{\xi}$ é finito para cada $k \in \omega$.

Seja $h_{\xi}: \omega \rightarrow \omega$ uma função crescente tal que 5) $A_{\xi} \backslash A_{k}^{\xi} \subseteq h_{\xi}(k)$, para cada $k \in \omega$.

Novamente pela seletividade do ultrafiltro $u_{\xi}$, existe $B_{\xi} \in u_{\xi}, B_{\xi} \subseteq A_{\xi}$ tal que 6) $B_{\xi} \cap h_{\xi}(1)=\emptyset$ e $\left|B_{\xi} \cap\left[h_{\xi}(k)+1, h_{\xi}(k+1)\right]\right| \leq 1, \forall k \in \omega$.

Seja $\left\{a_{k}^{\xi}: k \in \omega\right\}$ a enumeração crescente de $B_{\xi}$. 
Por 6) temos que $a_{k}^{\xi}>h_{\xi}(k)$ e $k<a_{k}^{\xi} \forall k \in \omega$. Portanto segue de 5) que $a_{k}^{\xi} \in A_{k}^{\xi}$, para cada $k \in \omega$ e para cada $\xi \in H$. Então pelo Lema 3.3 , podemos considerar, para cada $\dot{\xi} \in H$, um subconjunto $I_{\xi}$ de $\omega$ satisfazendo:

7) $\left\{a_{k}^{\xi}: k \in I_{\xi}\right\} \in u_{\xi} ; \forall \xi \in H$, e

8) $\left\{\left[k, a_{k}^{\xi}\right]: k \in \bigcup_{\xi \in H} I_{\xi}\right\}$ são dois a dois disjuntos.

Seja $\left\{i_{m}: m \in \omega\right\}$ uma enumeração crescente de $\bigcup_{\xi \in H} I_{\xi}$, e seja $r: \omega \rightarrow H$ definida por $r(m)=\xi \Leftrightarrow i_{m} \in I_{\xi}$. Observamos que a função $r$ está bem definida pois de 8 ) segue que se $\alpha, \beta \in H \operatorname{com} \alpha \neq \beta$ entào $I_{\alpha} \cap I_{\beta}=\emptyset$.

Para cada $m \in \omega$ definimos $b_{m}=a_{i_{m}}^{r(m)}$ e $E_{m}=F_{i_{m}}$.

(a) segue de $F_{0}=F$ e a definição de $E_{0}$.

(b) segue de $E=\bigcup_{k \in \omega} F_{k}$ e a definiçào de $E_{k}$, para cada $k \in \omega$.

Vamos verificar o item $(c)$ :

De s) temos que $b_{m}=a_{i_{m}}^{r(m)} \leq i_{m+1}-1$ e de 2) e a definiçào de $E_{m}$ temos que $E_{m}=F_{i_{m}} \subseteq F_{i_{m+1}-1}, \forall m \in \omega . \log 0$

$$
\begin{aligned}
& \bigcup\left\{\cup_{i \in S_{\xi}} \operatorname{supp}\left(f_{\xi}\left(i, b_{m}\right)\right):\{\xi\} \times S_{\xi} \in E_{m}\right\} \cup E_{m} \subseteq \\
& \subseteq \bigcup\left\{\cup_{i \in S_{\xi}} \operatorname{supp}\left(f_{\xi}(i, k)\right):\{\xi\} \times S_{\xi} \in F_{i_{m+1}-1}, k \leq i_{m+1}-1\right\} \cup F_{i_{m+1}-1} \subseteq \\
& \subseteq F_{i_{m+1}}=E_{m+1}, \quad \forall m \in \omega .
\end{aligned}
$$

(d) segue de $b_{m}=a_{i_{m}}^{r(m)} \in A_{i_{m}}^{r(m)}$ e da definiçào de $E_{m}$.

Por último, (e) segue de 7 ) e da definição da função $r$ e (f) da definição de $E_{m}$.

Lema 3.5. Sejam $x \in G,\left(g_{i}\right)_{i<p+1} \in(G)^{p+1} \in\left\{u_{\xi}: \omega \leq \xi<\mathfrak{c}\right\}$ uma familia de ultrafiltros seletivos incomparáveis sobre $\omega$.

Então existe um homomorfismo $\phi: G \rightarrow \mathbb{Z}(r)$ tal que: 
1) $\phi(x) \neq 0$, se $x \neq 0$;

2) $\left\{m \in \omega: \phi\left(f_{\xi}(k, m)\right)=\phi\left(x_{\xi, k}\right), \forall k \in S_{\xi}\right\} \in u_{\xi}$, para cada $\omega \leq \xi<\mathfrak{c}$,

3) $\left\{m \in \omega:\left(\phi\left(x_{m, k}\right)\right)_{k<p+1}=\left(\phi\left(g_{k}\right)\right)_{k<p+1}\right\}$ é finito.

Demonstração: Seja $F=\left(\bigcup_{i<p+1} \operatorname{supp}\left(g_{i}\right)\right) \cup \operatorname{supp}(x)$. Sejam $H \in(\mathfrak{c} \backslash \omega)^{\omega}, E$ um subconjunto enumerável de $\mathfrak{c} \times(p+1)$ tal que $(F \cup \omega \times(p+1)) \subseteq E, H \times(p+1) \subseteq E$, $\bigcup_{(k, m) \in S_{\xi} \times \omega} \operatorname{supp} f_{\xi}(k, m) \subseteq E, \forall \xi \in H$ e, para cada $\omega \leq \alpha<\mathfrak{c}$ tal que existe um $i<p+1 \operatorname{com}(\alpha, i) \in E$, tem-se $\{\alpha\} \times(p+1) \subseteq E$. Entào pelo lema 3.4 existem uma seqüencia crescente $\left\{b_{k}: k \in \omega\right\} \subseteq \omega$, uma função $r: \omega \rightarrow H$ e uma seqüência $\left\{E_{k}: k \in \omega\right\}$ de subconjuntos finitos de $E$ tais que:

(a) $F \subseteq E_{0}$

(b) $E=\bigcup_{k \in \omega} E_{k}$;

(c) $E_{k+1} \supseteq\left\{\bigcup_{i \in S_{\xi}} \operatorname{supp}\left(f_{\xi}\left(i, b_{k}\right)\right):\{\xi\} \times(p+1) \subseteq E_{k}\right\} \cup E_{k}$;

(d) $\left\{f_{r(k)}\left(i, b_{k}\right): i \in S_{r(k)}\right\} \cup\left\{x_{\alpha, i}:(\alpha, i) \in E_{k}\right\}$ é linearmente independente, para cada $k \in \omega$;

(e) $\left\{b_{k}: k \in r^{-1}(\{\xi\})\right\} \in u_{\xi}$, para cada $\xi \in H$;

(f) $E_{k} \cap(\omega \times(p+1))=N_{k} \times(p+1)$, para algum $N_{k} \in \omega$.

Primeiro definimos o homomorfismo $\phi$ em $\left\{x_{\alpha, i}:(\alpha, i) \in E\right\}$, por induçào.

Para cada $(\alpha, i) \in E_{0}$ definimos $\phi\left(x_{\alpha, i}\right)$ de forma que $\phi(x) \neq 0$.

Assumimos que $\phi$ já foi definida em $\left\{x_{\alpha, i}:(\alpha, i) \in E_{k}\right\}$ satisfazendo: para cada $0<t<k$

i) se $\{r(t)\} \times(p+1) \subseteq E_{t}$, então $\phi\left(f_{r(t)}\left(i, b_{t}\right)\right)=\phi\left(x_{r(t), i}\right), \forall i \in S_{r(t)}$ e

ii) $\left(\phi\left(x_{N, i}\right)\right)_{i<p+1} \neq\left(\phi\left(g_{i}\right)\right)_{i<p+1}, \forall N<N_{t+1}$.

Vamos estender o homomorfismo $\phi$ a $\left\{x_{\alpha, i}:(\alpha, i) \in E_{k+1}\right\}$ de forma que:

(I) se $\{r(k)\} \times(p+1) \subseteq E_{k}$ então $\phi\left(f_{r(k)}\left(i, b_{k}\right)\right)=\phi\left(x_{r(k), i}\right), \forall i \in S_{r(k)}$ e (II) $\left(\phi\left(x_{N, i}\right)\right)_{i<p+1} \neq\left(\phi\left(g_{i}\right)\right)_{i<p+1}, \forall N \in\left[N_{k}, N_{k+1}\right)$. 
Suponhamos que $\{r(k)\} \times(p+1) \subseteq E_{k}$, entào pela condição (c) temos que

$$
E_{k+1} \supseteq \bigcup_{i \in S_{r(k)}} \operatorname{supp}\left(f_{r(k)}\left(i, b_{k}\right)\right)
$$

e pela condição (f) temos que $E_{k+1} \cap \omega \times(p+1)=N_{k} \times(p+1)$.

Se $N_{k+1}=N_{k}+M$, vamos provar por induçào em $\left[N_{k}, N_{k+1}\right)$ que

Afrmação: existe, para cada $\eta<M, i_{N_{k}+\eta} \in(p+1)$ tal que $x_{N_{k}+\eta, i_{N_{k}+\eta}}$ satisfaz:

$$
\left\{f_{r(k)}\left(i, b_{k}\right): i \in S_{r(k)}\right\} \cup\left\{x_{N_{k}+\eta, i_{N_{k}+\eta}}: 0 \leq \eta<M\right\} \text { é linearmente independente. }
$$

$O$ conjunto $\left\{f_{r(k)}\left(i, b_{k}\right): i \in S_{r(k)}\right\}$ é linearmente independente e possui $\left|S_{r(k)}\right| \leq p$ elementos e o conjunto $\left\{x_{N_{k}, i}: i<p+1\right\}$ é linearmente independente com $(\mathrm{p}+1)$ elementos. Portanto existe $i_{N_{k}}<p+1$ tal que $\left\{f_{r(k)}\left(i, b_{k}\right): i \in S_{r(k)}\right\} \cup\left\{x_{N_{k}, i_{N_{k}}}\right\}$ é linearmente independente.

Suponhamos que $0<\eta<M-1 \mathrm{e}$

$$
\left\{f_{r(k)}\left(i, b_{k}\right): i \in S_{n(k)}\right\} \cup\left\{x_{N_{k}, i_{N_{k}}}, \ldots, x_{N_{k}+\eta, i_{N_{k}}}\right\} \text { él.i. }
$$

Observamos que o conjunto acima tem $\left|S_{r(k)}\right|+(\eta+1)$ elementos e que o conjunto

$$
\left\{x_{N_{k}, i_{N_{k}}}, \ldots, x_{N_{k}+\eta, i_{N_{k}+\eta}}\right\} \cup\left\{x_{N_{k}+(\eta+1), i}: i<p+1\right\}
$$

é li. de $(\eta+1)+(p+1)$ elementos. Portanto existe $i_{N_{k}+(\eta+1)} \in p+1$ tal que

$$
\left\{f_{r(k)}\left(i, b_{k}\right): i \in S_{r(k)}\right\} \cup\left\{x_{n_{k}, i_{N_{k}}}\right\} \cup\left\{x_{N_{k}+\eta+1, i_{N_{k}+(\eta+1)}}\right\}
$$

é linearmente independente, o que demonstra a afirmação.

Então podemos estender $\phi$ a $\left\{x_{\alpha, i}:(\alpha, i) \in E_{k+1}\right\}$ satisfazendo:

$$
\begin{aligned}
& \phi\left(f_{r(k)}\left(i, b_{k}\right)\right)=\phi(r(k), i), \forall i \in S_{r(k)} \mathrm{e} \\
& \phi\left(x_{N_{k}+\eta, i_{N_{k}+\eta}}\right) \neq \phi\left(g_{i_{N_{k}+\eta}}\right), \forall 0 \leq \eta<M .
\end{aligned}
$$


Logo $\phi$ satisfaz $(I)$ e $(I I)$.

Portanto temos definido o homomorfismo $\phi$ em $\left\{x_{\alpha, i}:(\alpha, i) \in E\right\}$ e como $\omega \times(p+1) \subseteq E$ a seqüência $\left(\phi\left(x_{m, i}\right)\right)_{i<p+1}$ está definida e satisfaz 3$)$.

Falta então definirmos $\phi$ em $\left\{x_{\alpha, i}:(\alpha, i) \in(\mathfrak{c} \times(p+1) \backslash E)\right\}$. Definimos $\phi$ neste conjunto por indução transfinita em $L=\mathfrak{c} \backslash\{\alpha:\{\alpha\} \times(p+1) \subseteq E\}$.

Seja $\gamma$ o menor ordinal no conjunto $L$ tal que o homomorfismo $\phi$ ainda não foi definido em $x_{\gamma, i}$ para todo $i<p+1$. Como $\phi\left(f_{\gamma}(i, m)\right)$ já foi definido $\forall i \in S_{\gamma}, \forall m \in \omega$, podemos definir, para cada $i \in S_{\gamma}, \phi\left(x_{\gamma, i}\right)$ tal que $\phi\left(x_{\gamma, i}\right)=u_{\gamma}-\lim _{m \rightarrow \infty}\left\{\phi\left(f_{\gamma}(i, m)\right): m \in \omega\right\}$. Se $i \in(p+1) \backslash S_{\gamma}$ definimos $\phi\left(x_{\gamma, i}\right)=0$.

Temos então $\phi: G \rightarrow \mathbb{Z}(r)$ homomorfismo satisfazendo 1), 2) e 3).

\section{Demonstração do Teorema 3.1:}

Pelo Lema 3.5, para cada $x \in G$ e para cada $(p+1)$-upla $\left(g_{i}\right)_{i<p+1} \in G^{p+1}$, existe um homomorfismo $\phi_{x,\left(g_{i}\right)_{i<p+1}}: G \rightarrow \mathbb{Z}(r)$ tal que:

1) $\phi_{x,\left(g_{i}\right)_{i<p+1}}(x) \neq 0$, se $x \neq 0$;

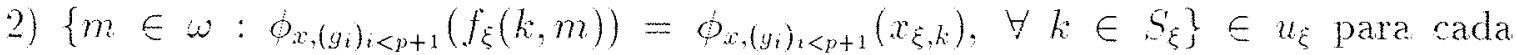
$\omega \leq \xi<\mathfrak{c}$,

3) $\left\{m \in \omega:\left(\phi_{x,\left(g_{i}\right)_{i<p+1}}\left(x_{m, k}\right)\right)_{k<p+1}=\left(\phi_{x,\left(g_{i}\right)_{i<p+1}}\left(g_{k}\right)\right)_{k<p+1}\right\}$ é finito.

Seja $\varphi: G \rightarrow \mathbb{Z}(r)^{c}$ a função diagonal

$$
\varphi=\triangle\left\{\phi_{x,\left(g_{i}\right)_{i<p+1}}: x \in G,\left(g_{i}\right) \in G^{p+1}\right\}
$$

$\varphi$ é um homomorfismo pois cada $\phi$ é um homomorfismo e $\varphi$ é injetor pelo item 1). Portanto $\varphi(G)$ é um subgrupo de $\mathbb{Z}(r)^{i}$ isomorfo a $G$. Vamos mostrar que $\varphi(G)$ com a topologia de subespaço de $\mathbb{Z}(r)^{c}$ é tal que $\varphi(G)^{p}$ é enumeravelmente compacto. Para isto, pelo Lema 3.2 é suficiente mostrar que cada seqüência $\left\{\left(\varphi\left(f_{\xi}(k, m)\right)\right)_{k \in S_{\xi}}: m \in\right.$ 
$\omega\}$ tem ponto de acumulação $\left(x_{\xi, k}\right)_{k \in S_{\xi}} \operatorname{em} \varphi(G)^{S_{\xi}}$.

Por 2) sabemos que a seqüència $\left\{\left(\phi_{x,\left(g_{i}\right)_{i<p+1}}\left(f_{\xi}(k, m)\right)\right)_{k \in s_{\xi}}: m \in \omega\right\}$ tem $u_{\xi}$-limite $\left(\phi_{x,\left(g_{i}\right)_{i<p+1}}\left(x_{\xi, k}\right)\right)_{i \in S_{\xi}}$. Portanto a seqüência $\left\{\left(\varphi\left(f_{\xi}(k, m)\right)\right)_{k \in S_{\xi}}: m \in \omega\right\}$ tem $u_{\xi}$-limite $\left(\varphi\left(x_{\xi, k}\right)_{k \in S_{\xi}} \operatorname{em}(\varphi(G))^{S_{\xi}}\right.$.

Falta mostrar que a seqüência $\left\{\left(\varphi\left(x_{m, k}\right)\right)_{k<p+1}: m \in \omega\right\}$ nào tem ponto de acumulação em $\varphi(G)^{p+1}$ do qual segue que $\varphi(G)^{p+1}$ não é enumeravelmente compacto.

Dada uma $(p+1)$-upla qualquer $\left(g_{i}\right)_{i<p+1}$ de elementos de $G$, temos que $\left(\varphi\left(g_{i}\right)\right)_{i<p+1}$ não é ponto de acumulação da seqüência $\left\{\left(\varphi\left(x_{m, k}\right)\right)_{k<p+1}: m \in \omega\right\}$ pois por 3$)$, para cada $x \in G,\left\{m \in \omega:\left(\phi_{x,\left(g_{i}\right)_{i<p+1}}\left(x_{m, k}\right)\right)_{k<p+1}=\left(\phi_{x,\left(g_{i}\right)_{i<p+1}}\left(g_{k}\right)\right)_{k<n+1}\right\}$ é finito.

\subsection{Compacidade enumerável e grupos de torção}

Nesta seção classificamos, sob a existência de $\mathfrak{c}$ ultrafiltros seletivos, os grupos de torção $G$ de cardinalidade $\mathfrak{c}$ que, para cada inteiro positivo $p$, admitem uma topologia de grupo topológico que faz $G^{p}$ enumeravelmente compacto e $G^{q+1}$ nâo enumeravelmente compacto para um $q>p$.

Teorema 3.6. Se existem $\mathfrak{c}$ ultrafiltros seletivos incomparáveis sobre w então para um grupo abeliano infinito de torção $G$, de cardinalidade no máximo $\mathfrak{c}$, as seguintes condiçôes säo equivalentes:

(a) para cada inteiro positivo $p$ existe $q>p$ tal que $G$ admite uma topologia de grupo topológico $(G, \tau)$ que satisfaz: $(G, \tau)^{p}$ é enumeravelmente compacto mas $(G, \tau)^{q+1}$ não é

enumeravelmente compacto;

(b) G admite uma topologia de grupo topológico $\tau$, enumeravelmente compacta tal que 
$(G, \tau)^{q}$ não é enumeravelmente compacto para algum $q>1$;

(c) G tem ordem limitada $n$ e $d G$ é finito ou tem cardinalidade $\mathfrak{c}$ para cada divisor próprio d de $n$;

(d) $G$ tem ordem limitada $n$ e para cada componente primária $G_{r}$ de $G$, a última invariante infinita de Ulm-Kaplansky de $G_{r}$ é igual a $\mathfrak{c}$.

Temos bastante trabalho prévio à demonstração do teorema. Primeiro vamos apresentar o $q$. Seja

$$
q=\left|\cup\left\{{ }^{F} n: F \in \mathcal{P}(p)\right\}\right|+1
$$

Sabemos que para um grupo abeliano $G$ como na condição $(d)$ do teorema, podemos achar um subgrupo finito $F$ de $G$ e um divisor $m$ de $n$ tais que $G \cong F \oplus$ $\oplus_{\left.d\right|_{m}} \mathbb{Z}(d)^{\left(\alpha_{d}\right)}$, com $\alpha_{m}=\mathfrak{c}$ e $\omega \leq \alpha_{d} \leq \mathfrak{c}$, para cada $\left.d\right|_{m}$. Como o produto de um grupo finito por um grupo enumeravelmente compacto é enumeravelmente compacto, podemos assumir $F=\{0\}$ e substituir $m$ por $n$. Assumimos então $G \cong \bigoplus_{\left.d\right|_{n}} \mathbb{Z}(d)^{\left(\alpha_{d}\right)}$, $\operatorname{com} \alpha_{n}=\mathfrak{c}$ e $\omega \leq \alpha_{d} \leq \mathfrak{c}$, para cada $\left.d\right|_{n}$.

Seja $\left\{x_{\alpha, i}: \alpha<\mathfrak{c}, i<q+1\right\}$ uma base para $\mathbb{Z}(n)^{(c)}$ (i.e um conjunto de geradores independentes de ordem $n$ ).

Suponhamos que a quantidade de divisores próprios de $n$, que aparecem na decomposição de $G$ em soma direta, é $h$. Enumeramos estes divisores por $d_{q+1}, \ldots, d_{q+h}$ e escrevemos $G \cong \mathbb{Z}\left(d_{q+1}\right)^{\left(\alpha_{1}\right)} \oplus \ldots \oplus \mathbb{Z}\left(d_{q+h}\right)^{\left(\alpha_{h}\right)} \cap Z(n)^{(\mathfrak{c})}$.

Para cada $1 \leq j \leq h$, seja $\left\{y_{\delta, q+j}: \delta<\alpha_{j}\right\}$ uma base para $\mathbb{Z}\left(d_{q+j}\right)^{\left(\alpha_{j}\right)}$.

Se $x$ é um elemento do grupo $G$, o suporte de $x$ denotado por supp(x) é um subconjunto finito de $\left(\bigcup_{j=1}^{h} \alpha_{j} \times\{q+j\}\right) \bigcup(\mathfrak{c} \times(q+1))$.

Enumeramos por $\left\{f_{\xi}: \omega \leq \xi<\mathfrak{c}\right\}$ as funçōes $f: S \times \omega \rightarrow G \operatorname{com} S \in[q+1] \leq p$ tais 
que para cada subconjunto finito $F$ de $\bigcup_{j=1}^{h}\left\{y_{\delta, q+j}: \delta<\alpha_{j}\right\} \cup\left\{x_{\alpha, i}: \alpha<\mathfrak{c}, i<q+1\right\}$, o conjunto $\omega \backslash\{m \in \omega: F \cup\{f(k, m): k \in S\}$ é linearmente independente $\}$ é finito. Chamamos de $S_{\xi}$ o elemento $S$ de $[q+1]^{\leq p}$ tal que $\operatorname{dom}\left(f_{\xi}\right)=S_{\xi} \times \omega$. Assumimos $\bigcup_{(k, m) \in \operatorname{dom}\left(f_{\xi}\right)} \operatorname{supp} f_{\xi}(k, m) \subseteq \xi \times(q+h+1)$.

Lema 3.7. Seja $\varphi: G \rightarrow \mathbb{Z}(n)^{\mathfrak{c}}$ um monomorfismo de grupos e seja $\left\{u_{\xi}: \omega \leq\right.$ $\xi<\mathfrak{c}\}$ uma família de ultrafiltros sobre $\omega$. Se para cada $\omega \leq \xi<\mathfrak{c}$, a seqüencia $\left\{\left(\varphi\left(f_{\xi}(i, m)\right)\right)_{i \in S_{\xi}}: m \in \omega\right\}$ tem $u_{\xi}-$ limite $\left(\varphi\left(c_{\xi, i} x_{\xi, i}\right)\right)_{i \in S_{\xi}} \in m \varphi(G)^{s_{\xi}}$, onde $c_{\xi, i} \in \mathbb{Z}$. então $\varphi(G)^{p}$ é enumeravelmente compacto como subespaço de $\mathbb{Z}(n)^{c}$.

Demonstração: Seja $\left\{\left(\varphi\left(a_{i, m}\right)\right)_{i<p}: m \in \omega\right\}$ uma seqüência em $\varphi(G)^{p}$.

Afrmação 1: Existem $S \subseteq p, c_{i} \in G$ e $E_{i} \subseteq S$ para cada $i<p, A \subseteq \omega$ infinito e subconjuntos $\left\{b_{i, m}: m \in \omega\right\}$ de $G$ para cada $i \in S$, tais que:

$\left\{\left(\varphi\left(b_{i, m}\right)\right)_{i \in S}: m \in A\right\}$ tem ponto de acumulação em $\varphi(G)^{S} \mathrm{e}$

$c_{i}=a_{i, m}-\sum_{j \in E_{i}} b_{j, m}$ para cada $m \in A$.

Demonstraçâo da Afirmação 1: Esta demonstração exige um pouco mais de atençào do que a feita na seção anterior pois agora já não temos mais um espaço vetorial. Seja u um ultrafiltro seletivo sobre $\omega$. Consideramos ultraproduto ${ }^{\omega} G / u$, que como vimos no capítulo anterior é um grupo abeliano de ordem $n$. Chamamos $R$ ao subgrupo de ${ }^{\omega} G / u$ gerado por $\left\{\left[\left\{a_{i, m}: m \in \omega\right\}\right]_{u}: i<p\right\}$. Isto é:

$$
R=\left\langle\left\{\left[\left\{a_{i, m}: m \in \omega\right\}\right]_{u}: i<p\right\}\right\rangle \text {. }
$$

Seja $C \subseteq G$ tal que $\left\{[\vec{x}]_{u}: x \in C\right\}$ é uma base para $R \cap\left\{[\vec{x}]_{u}: x \in G\right\}$. Sabemos que $R$ possui uma base que contém $\left\{[\vec{x}]_{u}: x \in C\right\}$, pois $R$ é um grupo abeliano de ordem finita e o subgrupo formado pelas classes das seqüências constante é puro. Como $R$ possui um conjunto de geradores de cardinalidade $p$ é de ordem finita, uma base 
para $R$ tem cardinalidade menor o igual a $p$.

Sejam então $S \subseteq p$ e $\left\{\left[\left\{b_{i, m}: m \in \omega\right\}\right]_{u}: i \in S\right\}$ tais que

(I) $\left\{\left[\left\{b_{i, m}: m \in \omega\right\}\right]_{u}: i \in S\right\} \bigcup\left\{[\vec{a}]_{u}: x \in C\right\}$ é uma base para $R$.

Escrevemos cada elemento do conjunto gerador original de $R$ como combinação linear dos elementos da base.

Para cada $i<p$, sejam $E_{i} \subseteq S$ e $C_{i} \subseteq C$ tais que

$$
\left[\left\{a_{i, m}: m \in \omega\right\}\right]_{u}=\sum_{x \in C_{i}} c_{i, x}[\vec{x}]_{u}+\sum_{j \in E_{i}} t_{j, i}\left[\left\{b_{j, m}: m \in \omega\right\}\right]_{u},
$$

onde $c_{i, x} \in \mathbb{Z} \forall x \in C_{i}$ e $t_{j, i} \in \mathbb{Z} \forall j \in E_{i}$.

Segue que a seqüência $\left\{a_{i, m}: m \in \omega\right\}-\sum_{j \in E_{i}}\left\{t_{j, i} \cdot b_{j, m}: m \in \omega\right\}$ é u-equivalente a uma seqüencia constante. Portanto existem $B \in u$ e elementos $x_{i}$ do grupo $G$ tais que para cada $i<p$

$$
x_{i}=\sum_{x \in C_{i}} c_{i, x} . x=a_{i, m}-\sum_{j \in E_{i}} t_{j, i} \cdot b_{j, m} \quad \forall m \in B .
$$

(II) Para cada $T \subseteq S$ nào vazio, e para cada conjunto $\left\{t_{i}: i \in T\right\} \subseteq \mathbb{Z}(n)$, se $\sum_{i \in T} t_{i}\left[\left\{b_{i, m}: m \in \omega\right\}\right]_{u} \neq 0$ então $\sum_{i \in T} t_{i}\left[\left\{b_{i, m}: m \in \omega\right\}\right]_{u}$ nào está na classe de $u-$ equivalência de uma seqüência constante. Isto é verdade pois

$$
\sum_{i \in T} t_{i}\left[\left\{b_{i, m}: m \in \omega\right\}\right]_{u} \in R
$$

e é uma combinação linear de elementos da base de $R$ que nào geram as constantes. Agora, para cada $T \subseteq S$ e para cada $\left\{t_{i}: i \in T\right\}$ que abreviamos por $\left\{t_{i}\right\}$ definimos a aplicação $h_{T,\left\{t_{i}\right\}}: \omega \rightarrow G$ por $h_{T,\left\{t_{i}\right\}}(m)=\sum_{i \in T} t_{i} \cdot b_{i, m}$.

Se $\sum_{i \in T} t_{i}\left[\left\{b_{i, m}: m \in \omega\right\}\right]_{u} \neq 0$, por $(I I)$ e por ser $u$ seletivo podemos afirmar que existe $A_{T,\left\{t_{i}\right\}} \in u, A_{T,\left\{t_{i}\right\}} \subseteq B$, tal que a aplicação $\left.h_{T,\left\{t_{i}\right\}}\right|_{A_{T,\left\{t_{i}\right\}}}$ é injetora. Como só temos uma quantidade finita de funções $h_{T,\left\{t_{i}\right\}}$, 
(III) existe $A \in u, A \subseteq B$ tal que $\left.h_{T,\left\{t_{\ell}\right\}}\right|_{A}$ é injetora, para cada $T \subseteq S$ e para cada $\left\{t_{i}: i \in \mathbb{Z}(n)\right\}$.

Seja $\left\{m_{k}: k \in \omega\right\}$ uma enumeração de $A$ e seja a função $f: S \times \omega \rightarrow G$ definida por $f(i, k)=b_{i, m_{k}} \forall i \in S, \forall k \in \omega$.

Afrrmação: existe $\omega \leq \xi<\omega$, tal que $f_{\xi}=f$.

Para demonstrar esta afirmaçào, precisamos provar que para cada subconjunto finito $F$ de $\bigcup_{j=1}^{h}\left\{y_{\delta, p+j}: \delta<\alpha_{j}\right\} \cup\left\{x_{\alpha, i}: \alpha<\mathfrak{c}, i<q+1\right\}$, o conjunto

$\omega \backslash\{k \in \omega: F \cup\{f(i, k): i \in S\}$ é linearmente independente $\}$ é finito.

Seja entào $F$ um subconjunto finito de $\bigcup_{j=1}^{h}\left\{y_{\delta, q+j}: \delta<\alpha_{j}\right\} \cup\left\{x_{\alpha, i}: \alpha<\mathfrak{c}, i<q+1\right\}$, e vamos supor, por absurdo, que o conjunto

$$
X=\omega \backslash\{k \in \omega: F \cup\{f(i, k): i \in S\} \text { é linearmente independente }\} \text { é infinito. }
$$

Entào, para cada $k \in X$, existem $F_{k} \subseteq F, \emptyset \neq T_{k} \subseteq S$ e inteiros $t_{i}^{k}$ para cada $i \in S$ tais que $\sum_{i \in T_{k}} t_{i}^{k} \cdot b_{i, m_{k}}$ é combinaçào linear dos elementos de $F_{k}$. Como $F$ é finito, $S$ também é finito e os escalares (inteiros) que aparecem nas combinaçōes lineares são elementos de $\mathbb{Z}(n)$ que também é finito, temos que existem um subconjunto infinito $Y$ de $X, E \subseteq F, T \subseteq S$ e inteiros $\left\{t_{i}: i \in T\right\}$ tais que $F_{k}=E, T_{k}=T$, $\left\{t_{i}^{k}: i \in T_{k}\right\}=\left\{t_{i}: i \in T\right\}$ para todo $k \in Y$ e $\left\{\sum_{i \in T} t_{i} \cdot b_{i, m_{k}}: k \in Y\right\}$ é unitário. Mas isto contradiz $(I I I)$. Portanto a suposição é falsa e temos demonstrada a afirmação.

Por hipótese temos que $\left\{\varphi\left(f_{\xi}(i, k)\right)_{i \in S_{\xi}}: k \in \omega\right\}$ tem $u_{\xi}$ limite $\left(\varphi\left(c_{\xi, i} \cdot x_{\xi, i}\right)\right)_{i \in S_{\xi}} \mathrm{em}$ $\varphi(G)^{S_{\xi}}$. Segue que $\left\{\left(\varphi\left(b_{i, m}\right)\right)_{i \in S}: m \in A\right\}$ possui ponto de acumulação em $(\varphi(G))^{S}$ e que $c_{i}=a_{i, m}-\sum_{j \in E_{3}} b_{j, m}$ para cada $m \in A$.

Afirmação 2: A seqüência $\left\{\varphi\left(a_{i, m}\right)_{i<p}: m \in \omega\right\}$ possui ponto de acumulaçào em 
$\varphi(G)^{p}$

Análogo a Afirmação 2 do Lema 3.2.

Lema 3.8. Sejam $H$ um subconjunto infinito enumerável de $\mathfrak{c} \backslash \omega, \quad\left\{u_{\xi}: \xi \in H\right\}$ conjunto de ultrafiltros seletivos incomparáveis de $\omega$ e $F$ um subconjunto finito de $\left(\bigcup_{j=1}^{h} \alpha_{j} \times\{q+j\}\right) \bigcup(\mathfrak{c} \times(q+1))$.

Seja E um subconjunto enumerável de $\left(\bigcup_{j=1}^{h} \alpha_{j} \times\{q+j\}\right) \bigcup(\mathfrak{c} \times(q+1))$ tal que $F \cup(\omega \times(q+1)) \subseteq E, \quad H \times(q+1) \subseteq E \quad \epsilon \quad \bigcup_{(k, m) \in S_{\xi} \times \omega} \operatorname{supp}\left(f_{\xi}(k, m)\right) \subseteq E$, para cada $\xi \in H$.

Entäo existem uma seqüência crescente $\left\{b_{k}: k \in \omega\right\} \subseteq \omega$, uma funçào $r: \omega \rightarrow H \in$ uma seqüência $\left\{E_{k}: k \in \omega\right\}$ de subconjuntos finitos de $E$ tais que:

(a) $F \subseteq E_{0}$;

(b) $E=\bigcup_{k \in \omega} E_{k}$;

(c) $E_{k+1} \supseteq\left\{\bigcup_{i \in S_{\xi}} \operatorname{supp}\left(f_{\xi}\left(i, b_{k}\right)\right):\{\xi\} \times(q+1) \subseteq E_{k}\right\} \cup E_{k}$ :

(d) $\left\{f_{r(k)}\left(i, b_{k}\right): i \in S_{r(k)}\right\} \bigcup\left\{x_{\alpha, i}:(\alpha, i) \in E_{k}\right\} \bigcup\left\{y_{\delta, q+j}:(\delta, q+j) \in E_{k}, 1 \leq j \leq h\right\}$

é linearmente independente, para cada $k \in \omega$;

(e) $\left\{b_{k}: k \in r^{-1}(\{\xi\})\right\} \in u_{\xi}$, para cada $\xi \in H$;

(f) $E_{k} \cap \omega \times(q+1)=N_{k} \times(q+1)$, para algum $N_{k} \in \omega$.

Demonstração: Definimos por indução uma seqüência $\left\{F_{k}: k \in \omega\right\}$ de subconjuntos finitos de $E$, como segue:

1) $F_{0}=F$

2) $F_{k+1} \in E^{<\omega}$ tal que

$F_{k+1} \supseteq F_{k} \cup \bigcup_{m \leq k}\left\{\bigcup_{i \in S_{\xi}} \operatorname{supp}\left(f_{\xi}(i, m)\right):\{\xi\} \times(q+1) \subseteq F_{k}\right\}$ e se $m \in \omega$ e existe $i<q+1$ tal que $(m, i) \in F_{k+1}$, então $(m+1) \times(q+1) \subseteq F_{k+1}$;

3) $E=\bigcup_{k \in \omega} F_{k}$.

Para carla $\xi \in H$ e para cada $k \in \omega$, seja 
$A_{k}^{\xi}=\left\{m \in \omega:\left\{f_{\xi}(i, m): i \in S_{\xi}\right\} \cup\left\{x_{\alpha, i}:(\alpha, i) \in F_{k}\right\} \cup\left\{y_{\delta, q+j}:(\delta, q+j) \in F_{k}, 1 \leq\right.\right.$ $j \leq h\}$ é l.i. $\}$.

Pela definição de $f_{\xi}$, temos que $A_{k}^{\xi} \in u_{\xi}, \forall k \in \omega, \forall \xi \in H$.

Como $u_{\xi}$ é ultrafiltro seletivo, é também $P$-ponto. Logo existe $A_{\xi} \in u_{\xi}$ que satisfaz 4) $A_{\xi} \backslash A_{k}^{\xi}$ é finito para cada $k \in \omega$.

Seja $h_{\xi}: \omega \rightarrow \omega$ uma função crescente tal que

5) $A_{\xi} \backslash A_{k}^{\xi} \subseteq h_{\xi}(k)$, para cada $k \in \omega$.

Novamente pela seletividade do ultrafiltro $u_{\xi}$, existe $B_{\xi} \in u_{\xi}, B_{\xi} \subseteq A_{\xi}$ tal que

6) $B_{\xi} \cap h_{\xi}(1)=\emptyset$ e $\left|B_{\xi} \cap\left[h_{\xi}(k)+1, h_{\xi}(k+1)\right]\right| \leq 1, \forall k \in \omega$.

Seja $\left\{a_{k}^{\alpha}: k \in \omega\right\}$ a enumeração crescente de $B_{\xi}$.

Por 6) temos que $a_{k}^{\xi}>h_{\xi}(k)$ e $k<a_{k}^{\xi} \forall k \in \omega$. Portanto segue de 5) que $a_{k}^{\xi} \in A_{k}^{\xi}$, para cada $k \in \omega$ e para cada $\xi \in H$. Entào pelo Lema 3.3, podemos considerar, para cada $\xi \in H$, um subconjunto $I_{\xi}$ de $\omega$ satisfazendo:

7) $\left\{a_{k}^{\xi}: \not{k} \in I_{\xi}\right\} \in u_{\xi}, \forall \xi \in H, \mathrm{e}$

8) $\left\{\left[k, a_{k}^{\xi}\right]: k \in \bigcup_{\xi \in H} I_{\xi}\right\}$ são dois a dois disjuntos.

Seja $\left\{i_{m}: m \in \omega\right\}$ uma enumeraçàc crescente de $\bigcup_{\xi \in H} I_{\xi}$, e seja $r: \omega \rightarrow H$ definida por $r(m)=\xi \Leftrightarrow i_{m} \in I_{\xi}$. Observamos que a função $r$ está bem definida pois de 8) segue que se $\alpha, \beta \in H \operatorname{com} \alpha \neq \beta$ então $I_{\alpha} \cap I_{\beta}=\emptyset$.

Para cada $m \in \omega$ definimos $b_{m}=a_{i_{m}(m)}^{r(m)}$ e $E_{m}=F_{i_{m}}$.

(a) segue de $F_{0}=F$ e a definição de $E_{0}$.

(b) segue de $E=\bigcup_{k \in \omega} F_{k}$ e a definiçào de $E_{k}$, para cada $k \in \omega$.

Vamos verificar o item $(c)$ :

De 8) temos que $b_{m}=a_{i_{m}}^{r(m)} \leq i_{n+1}-1$ e de 2) e a definiçào de $E_{m}$ temos que 


$$
\begin{aligned}
E_{m}= & F_{i_{m}} \subseteq F_{i_{m+1}-1}, \forall m \in \omega . \text { Logo } \\
& \bigcup\left\{\cup_{i \in S_{\xi}} \operatorname{supp}\left(f_{\xi}\left(i, b_{m}\right)\right):\{\xi\} \times S_{\xi} \subseteq E_{m}\right\} \cup E_{m} \subseteq \\
& \subseteq \bigcup\left\{\cup_{i \in S_{\xi}} \operatorname{supp}\left(f_{\xi}(i, k)\right):\{\alpha\} \times S_{\xi} \subseteq F_{i_{m+1}-1}, k \leq i_{m+1}-1\right\} \cup F_{i_{m+1}-1} \subseteq \\
& \subseteq F_{i_{m+1}}=E_{m+1}, \quad \forall m \in \omega .
\end{aligned}
$$

(d) segue de $b_{m}=a_{i_{m}}^{r(m)} \in A_{i_{m}}^{r(m)}$ e da definiçào de $E_{m}$.

Por último, (e) segue de 7 ) e da definição da função $r$ e (f) segue da definição de $E_{m}$. $\square$

Observação 3.9. Como para cada $\omega \leq \xi<\mathfrak{c}, \mid\left\{f_{\xi}\left((k, m): k \in S_{\xi}, m \in \omega\right\} \mid \leq \omega \mathrm{e}\right.$ $\operatorname{ord}\left(f_{\xi}(k, m)\right) \leq n$, se $u_{\xi}$ é um ultrafiltro seletivo sobre $\omega$ então existe um divisor $d_{\xi, k}$ de $n$ e $B_{\xi, k} \in u_{\xi}$ tais que $\left\{m \in \omega: \operatorname{ord}\left(f_{\xi}(k, m)\right)=d_{\xi, k}\right\}=B_{\xi, k}$. Se $B_{\xi}=\bigcap_{k \in S_{\xi}} B_{\xi, k}$, segue que para todo $m \in B_{\xi}$ vale $\operatorname{ord}\left(f_{\xi}(k, m)\right)=d_{\xi, k}$ e $B_{\xi} \in u_{\xi}$.

Seja $c_{\xi, k}$ um inteiro que satisfaz $c_{\xi, k} \cdot d_{\xi, k}=n$.

Agora estamos en condições de enunciar o próximo lema.

Lema 3.10. Sejam $x \in G,\left(g_{i}\right)_{i<q+1} \in G^{q+1} \in\left\{u_{\xi}: \omega \leq \xi<\mathfrak{c}\right\}$ uma família de ultrafiltros seletivos incomparáveis sobre $\omega$.

Então existe um homomorfismo $\phi: G \rightarrow \mathbb{Z}(n)$ tal que:

1) $\phi(x) \neq 0$, se $x \neq 0$;

2) $\left\{m \in \omega: \phi\left(f_{\xi}(k, m)\right)=\phi\left(c_{\xi, k}, x_{\xi, k}\right), \forall k \in S_{\xi}\right\} \in u_{\xi}$, para cada $\omega \leq \xi<\mathfrak{c}$,

3) $\left\{m \in \omega:\left(\phi\left(x_{m, k}\right)\right)_{k<q+1}=\left(\phi\left(g_{k}\right)\right)_{k<q+1}\right\}$ é finito.

Demonstração: Seja $F=\bigcup_{i<q+1} \operatorname{supp}\left(g_{i}\right) \cup \operatorname{supp}(x)$. Sejam $H \in(\mathfrak{c} \backslash \omega)^{\omega}, E$ um subconjunto enumerável de $\left(\bigcup_{j=1}^{h} \alpha_{j} \times\{q+j\}\right) \cup(\mathfrak{c} \times(q+1))$ tal que $F \cup \omega \times(q+1) \subseteq E$, $H \times(q+1) \subseteq E, \bigcup_{(k, m) \in S_{\xi} \times \omega} \operatorname{supp} f_{\xi}(k, m) \subseteq E$, para cada $\xi \in H$ e para cada $\omega \leq \xi<\mathfrak{c}$ tal que existe um $i<q+1 \operatorname{com}(\xi, i) \in E$ tem-se $\{\xi\} \times(q+1) \subseteq E$. 
Então pelo Lema 3.8 existem uma seqüência crescente $\left\{b_{k}: k \in \omega\right\} \subseteq \omega$, uma função $r: \omega \rightarrow H$ e uma seqüência $\left\{E_{k}: k \in \omega\right\}$ de subconjuntos finitos de $E$ tais que:

(a) $F \subseteq E_{0}$;

(b) $E=\bigcup_{k \in \omega} E_{k}$;

(c) $E_{k+1} \supseteq\left\{\bigcup_{i \in S_{\xi}} \operatorname{supp}\left(f_{\xi}\left(i, b_{k}\right)\right):\{\xi\} \times(q+1) \subseteq E_{k}\right\} \cup E_{k}$;

(d) $\left\{f_{r(k)}\left(i, b_{k}\right): i \in S_{r(k)} \cup\left\{x_{\alpha, i}:(\alpha, i) \in E_{k}\right\} \cup\left\{y_{\delta, q+j}:(\delta, q+j) \in E_{k}, 1 \leq j \leq h\right\}\right.$ é linearmente independente, para cada $k \in \omega$;

(e) $\left\{b_{k}: k \in r^{-1}(\{\xi\})\right\} \in u_{\xi}$, para cada $\xi \in H$;

(f) $E_{k} \cap \omega \times(q+1)=N_{k} \times(q+1)$, para algum $N_{k} \in \omega$.

Primeiro definimos o homomorfismo $\phi$ em $\left\{y_{\delta, q+j}:(\delta, q+j) \in E\right\} \bigcup\left\{x_{\alpha, i}:(\alpha, i) \in E\right\}$, por indução.

Para cada $(\delta, q+j) \in E_{0}$ e para cada $(\alpha, i) \in E_{0}$ definimos $\phi\left(y_{\delta, q+j}\right)$ e $\phi\left(x_{\alpha, i}\right)$ de forma que $\phi(x) \neq 0$.

Assumimos que $\phi$ já foi definida em $\left\{y_{\delta, q+j}:(\delta, q+j) \in E_{k}\right\} \bigcup\left\{x_{\alpha, i}:(\alpha, i) \in E_{k}\right\}$ satisfazendo: para cada $0<t<k$

i) se $\{r(t)\} \times S_{r(t)} \subseteq E_{t}$, então $\phi\left(f_{r(i)}\left(i, b_{t}\right)\right)=\phi\left(c_{r(t), i} x_{r(t), i}\right), \forall i \in S_{r(t)}$ e

ii) $\left(\phi\left(x_{N, i}\right)\right)_{i<q+1} \neq\left(\phi\left(g_{i}\right)\right)_{i<q+1}, \forall N<N_{t+1}$.

Vamos estender o homomorfismo $\phi$ a $\left\{y_{\delta, q+j}:(\delta, q+j) \in E_{k+1}\right\} \bigcup\left\{x_{\alpha, i}:(\alpha, i) \in E_{k+1}\right\}$ de forma que:

(I) se $\{r(k)\} \times S_{r(k)} \subseteq E_{k}$ então $\phi\left(f_{r(k)}\left(i, b_{k}\right)\right)=\phi\left(c_{r(k), i}, x_{r(k), i}\right), \forall i \in S_{r(k)}$ e (II) $\left(\phi\left(x_{N, i}\right)\right)_{i<q+1} \neq\left(\phi\left(g_{i}\right)\right)_{i<q+1}, \forall N \in\left[N_{k}, N_{k+1}\right)$.

Suponhamos que $\{r(k)\} \times(q+1) \subseteq E_{k}$, então pela condição $(\mathrm{c})$ temos que $E_{k+1} \supseteq \bigcup_{i \in S_{r(k)}} \operatorname{supp}\left(f_{r(k)}\left(i, b_{k}\right)\right)$ e pela condição $(f)$ temos que $E_{k+1} \cap \omega \times(p+1)=$ $N_{k+1} \times(q+1)$.

Se $N_{k+1}=N_{k}+M$, vamos provar por indução em $\left[N_{k}, N_{k+1}\right)$ que 
Afirmação: existe, para cada $\eta<M, i_{N_{k}+\eta} \in(q+1)$ tal que $x_{N_{k}+\eta, i_{N_{k}+\eta}}$ satisfaz: $\left\{f_{r(k)}\left(i, b_{k}\right): i \in S_{r(k)}\right\} \cup\left\{x_{N_{k}+\eta, i_{N_{k}+\eta}}: 0 \leq \eta<M\right\}$ é linearmente independente.

Suponhamos por absurdo que, para cada $i<q+1$, existe $F_{i} \in \mathcal{P}\left(S_{r(k)}\right), a_{l, i} \in n, \forall l \in$ $F_{i}$ e $\theta_{i} \in n, \theta_{i} \neq 0$ tais que

$$
\sum_{l \in F_{i}} a_{l, i} f_{r(k)}\left(l, b_{k}\right)=\theta_{i} \cdot x_{N_{k}, i}
$$

Como temos menos que $(q+1)$ combinações lineares possiveis deste tipo, segue que existem $i, j<q+1 \operatorname{com} i \neq j$ tais que

$$
\theta_{i}, x_{N_{k}, i}=\sum_{l \in F_{i}} a_{l, i} f_{r(k)}\left(l, b_{k}\right)=\sum_{l \in F_{3}} a_{l, j} f_{r(k)}\left(l, b_{k}\right)=\theta_{j} \cdot x_{N_{k}, j}
$$

Mas isto não é possível já que $x_{N_{k}, i}$ e $x_{N_{k}, j}$ são independentes, portanto existe $i_{N_{k}}<q+1$ tal que $\left\{f_{r(k)}\left(i, b_{k}\right): i \in S_{r(k)}\right\} \cup\left\{x_{N_{k}, i_{N_{k}}}\right\}$ é independente.

Suponhamos que $0<\eta<M-1$ e

$$
\left\{f_{r(k)}\left(i, b_{k}\right): i \in S_{r(k)}\right\} \cup\left\{x_{N_{k}, i_{N_{k}}}, \ldots, x_{N_{k}+\eta, i_{N_{k}+\eta}}\right\} \text { é l.i. }
$$

Seja $\left\{x_{N_{k}+\eta+1, i}: i<q+1\right\}$ e suponhamos por absurdo que para cada $i<q+1$, existe $F_{i} \in\left(S_{r(k)}\right), a_{l, i} \in n, \forall l \in F_{i}, \gamma_{s, i} \in n, \forall 0 \leq s \leq \eta$ e $\theta_{i} \in n$ tais que

$$
\sum_{l \in F_{i}} a_{l, i} f_{s(k)}\left(l, b_{k}\right)=\theta_{i} \cdot x_{N_{k}+n+1, i}-\sum_{s=0}^{\eta} \gamma_{s, i} \cdot x_{N_{k}+s, i_{N_{k}+s}} .
$$

De novo, temos menos que $(q+1)$ possibilidades para o lado esquerdo da igualdade, portanto existem $i, j<q+1, i \neq j$ tais que

$$
\theta_{i} \cdot x_{N_{k}+\eta+1, i}-\sum_{s=0}^{\eta} \gamma_{s, i} \cdot x_{N_{k}+s, i_{N_{k}+s}}=\theta_{j} \cdot x_{N_{k}+\eta+1, j}-\sum_{s=0}^{\eta} \gamma_{s, j} \cdot x_{N_{k}+s, i_{N_{k}+s}}
$$


o que é absurdo pois

$$
\left\{x_{N_{k}+\eta+1, i}, x_{N_{k}+\eta+1, j}\right\} \cup\left\{x_{N_{k}+s, i_{N_{k}+s}}: 0 \leq s \leq \eta\right\}
$$

é linearmente independente.

Portanto existe $i_{N_{k}+\eta+1}<q+1$ tal que

$$
\left\{f_{r(k)}\left(i, b_{k}\right): i \in S_{r(k)}\right\} \cup\left\{x_{N_{k}, i_{N_{k}}}, \ldots, x_{N_{k}+\eta, i_{N_{k}+\eta}}, x_{N_{k}+\eta+1, i_{N_{k}+\eta+1}}\right\} \text {, é l.i.. }
$$

Então temos que

$$
\left\{f_{r(k)}\left(i, b_{k}\right): i \in S_{r(k)}\right\} \cup\left\{x_{N_{k}+\eta, i_{N_{k}+\eta}}: 0 \leq \eta<M\right\}
$$

é linearmente independente. Logo podemos estender o homomorfismo $\phi$ a

$$
\left\{y_{\delta, q+j}:(\delta, q+j) \in E_{k+1}\right\} \bigcup\left\{x_{\alpha, i}:(\alpha, i) \in E_{k+1}\right\}
$$

satisfazendo:

$\phi\left(f_{r(k)}\left(i, b_{k}\right)\right)=\phi\left(c_{r(k), i} \cdot x_{r(k), i}\right), \forall i \in S_{r(k)} \mathrm{e}$

$\left(\phi\left(x_{N_{k}+\eta, i_{N_{k}+\eta}}\right)\right) \neq\left(\phi\left(g_{i_{N_{k}+\eta}}\right)\right), \forall 0 \leq \eta<M$, e $\phi$ verifica $(I)$ e $(I I)$.

Portanto temos definido o homomorfismo $\phi \mathrm{em}$

$$
\left\{y_{\delta, q+j}:(\delta, q+j) \in E\right\} \bigcup\left\{x_{\alpha, i}:(\alpha, i) \in E\right\}
$$

e como $\omega \times(q+1) \subseteq E$ a seqüência $\left(\phi\left(x_{m, i}\right)\right)_{i<q+1}$ está definida e satisfaz 3$)$.

Para cada $1 \leq j \leq h$, se $(\delta, q+j) \in \alpha_{j} \times\{q+j\} \backslash E$, definimos $\phi\left(y_{\hat{j}, p+j}\right)=0$. Falta então definirmos $\phi \operatorname{em}\left\{x_{\alpha, i}:(\alpha, i) \in(\mathfrak{c} \times(q+1) \backslash E)\right\}$. Definimos $\phi$ neste conjunto por indução transfinita em $L=\mathfrak{c} \backslash\{\alpha:\{\alpha\} \times(q+1) \subseteq E\}$.

Seja $\gamma$ o mínimo ordinal no conjunto $L$ tal que o homomorfismo $\phi$ ainda näo foi definido em $x_{\gamma, i}$ para todo $i<q+1$. Como $\phi\left(f_{\gamma}(i, m)\right)$ já foi definido $\forall i \in S_{\gamma}$, $\forall m \in \omega$, podemos definir, para cada $i \in S_{\gamma}, \phi\left(x_{\gamma, i}\right)$ tal que

$$
\phi\left(c_{\gamma, i} x_{\gamma, i}\right)=u_{\gamma}-\lim \left\{\phi\left(f_{\gamma}(i, m)\right): m \in \omega\right\} .
$$


Se $i \in(q+1) \backslash S_{\gamma}$ definimos $\phi\left(x_{\gamma, i}\right)=0$.

Temos então $\phi: G \rightarrow \mathbb{Z}(n)$ homomorfismo satisfazendo 1), 2) e 3).

Vamos então demonstrar o Teorema 3.6.

\section{Demonstração do Teorema 3.6:}

(a) $\Rightarrow(b)$ é obvia.

$(b) \Rightarrow(c)$ foi justificada na demonstração do Corolário 1.38

$(c) \Rightarrow(d)$ está justificada no Lema 1.6.

Todas as implicações acima são provadas em ZFC.

Vamos provar $(d) \Rightarrow(a)$. É para provar esta implicação que precisamos assumir a existência dos ultrafiltros seletivos.

Pelo Lema 3.10, para cada $x \in G$ e para cada $(q+1)$-upla $\left(g_{i}\right)_{i<q+1} \in G^{q+1}$, existe um homomorfismo $\phi_{x,\left(g_{i}\right)_{i<q+1}}: G \rightarrow \mathbb{Z}(n)$ tal que:

1) $\phi_{x,\left(g_{i}\right)_{i<q+1}}(x) \neq 0$, se $x \neq 0$;

2) $\left\{m \in \omega: \phi_{x,\left(g_{2}\right)_{i<q+1}}\left(f_{\xi}(k, m)\right)=\phi_{x,\left\{y_{1}\right)_{i<q+1}}\left(c_{\hat{\xi}, k} x_{\hat{\zeta}, k}\right), \forall k \in S_{\xi}\right\} \in u_{\xi}$ para cada $\omega \leq \xi<\mathfrak{c}$,

3) $\left\{m \in \omega:\left(\phi_{x,\left(g_{2}\right)_{i<q+1}}\left(x_{m, k}\right)\right)_{k<q+1}=\left(\phi_{x,\left(g_{i}\right)_{i<q+1}}\left(g_{k}\right)\right)_{k<p+1}\right\}$ é finito.

Seja $\varphi: G \rightarrow \mathbb{Z}(n)^{\mathfrak{c}}$ a função diagonal

$$
\varphi=\triangle\left\{\phi_{x,\left(g_{i}\right)_{i<p+1}}: x \in G,\left(g_{i}\right) \in G^{q+1}\right\}
$$

$\varphi$ é um homomorfismo pois cada $\phi$ é um homomorfismo e $\varphi$ é injetor pelo item 1). Portanto $\varphi(G)$ é um subgrupo de $\mathbb{Z}(n)^{c}$ isomorfo a $G$. Vamos mostrar que $\varphi(G)$ com a topologia de subespaço de $\mathbb{Z}(n)^{c}$ é tal que $\varphi(G)^{p}$ é enumeravelmente compacto. Para 
isto, pelo Lema 3.7 é suficiente mostrar que cada seqüência

$$
\left\{\left(\varphi\left(f_{\xi}(k, m)\right)\right)_{k \in S_{\xi}}: m \in \omega\right\}
$$

tem ponto de acumulação $\left(c_{\xi, k} \cdot x_{\xi, k}\right)_{k \in S_{\xi}}$ em $\varphi(G)^{S_{\xi}}$.

Por 2) sabemos que a seqüência $\left\{\left(\phi_{x,\left(g_{i}\right)_{i<q+1}}\left(f_{\alpha}(k, m)\right)\right)_{k \in S_{\alpha}}: m \in \omega\right\}$ tem $u_{\xi}$-limite $\left(\phi_{x,\left(g_{i}\right)_{i<q+1}}\left(c_{\xi, k} x_{\xi, k}\right)\right)_{i \in S_{\xi}}$. Portanto a seqüência $\left\{\left(\varphi\left(f_{\xi}(k, m)\right)\right)_{k \in S_{\xi}}: m \in \omega\right\}$ tem $u_{\xi^{-}}$ limite $\left(\varphi\left(c_{\xi, k}, x_{\xi, k}\right)_{k \in S_{\xi}} \operatorname{em}(\varphi(G))^{S_{\xi}}\right.$.

Falta mostrar que a seqüência $\left\{\left(\varphi\left(x_{m}, k\right)\right)_{k<q+1}: m \in \omega\right\}$ não tem ponto de acumulaçào em $\varphi(G)^{q+1}$ do qual segue que $\varphi(G)^{q+1}$ nào é enumeravelmente compacto. Dada uma $(q+1)$-upla qualquer $\left(g_{i}\right)_{i<q+1}$ de elementos de $G$, temos que $\left(\varphi\left(g_{i}\right)\right)_{i<q+1}$ não é ponto de acumulação da seqüência $\left\{\left(\varphi\left(x_{m}, k\right)\right)_{k<q+1}: m \in \omega\right\}$ pois por 3$)$, para cada $x \in G,\left\{m \in \omega:\left(\phi_{x,\left(g_{i}\right)_{i<q+1}}\left(x_{m, k}\right)\right)_{k<q+1}=\left(\phi_{x,\left(g_{i}\right)_{k<q+1}}\left(g_{k}\right)\right)_{k<q+1}\right\}$ é finito. 


\section{Capítulo 4}

\section{Um grupo abeliano livre}

\section{enumeravelmente compacto cujo tamanho tem cofinalidade enumerável}

Neste capítulo apresentamos a construção, usando forcing, de um grupo abeliano livre enumeravelmente compacto cuja cardinalidade pode ter cofinalidade enumerável.

Exemplo 4.1. É consistente com ZFC que $2^{\mathfrak{C}}$ é "arbitrariamente grande" e que existe uma topologia de grupo, enumeravelmente compacta, sem seqüencias convergentes, no grupo livre de cardinalidade $\lambda$ para todo $\lambda \in\left[\mathfrak{c}, 2^{\mathfrak{c}}\right]$. Em particular, é consistente que existem topologias de grupo enumeravelmente compactas num grupo abeliano livre cuja cardinalidade tem cofinalidade enumerável.

Vamos construir, usando forcing, um subgrupo $G$ de $\mathbb{T}^{\circ}$ de tamanho $\kappa$ que é 
algebricamente livre e enumeravelmente compacto com a topologia de subespaço. De fato, vamos construir um subconjunto livre

$$
X=\left\{x_{\beta}: \beta<\kappa\right\} \text { de } \mathbb{T}^{\mathfrak{c}}
$$

com $\kappa \geq \omega_{2}, \kappa$ regular, tal que para cada $\gamma \in[\mathfrak{c}, \kappa)$, o grupo gerado por $\left\{x_{\beta}: \beta<\gamma\right\}$ seja enumeravelmente compacto.

Cada elemento do grupo $G$ se escreve como combinação linear finita de elementos do conjunto $X$. Então, para cada $x \in G$ existe um conjunto $E \in[\kappa]<\omega$ e uma função

$$
f: E \rightarrow \mathbb{Z} \backslash\{0\} \text { tal que } x=\sum_{\xi \in E} f(\xi) x_{\xi}
$$

Vemos que a funçào $f$ define o elemento $x$ por isso vamos chamar $f$ de representaçấo de $x$.

Queremos que $X$ seja um conjunto livre, isto é, que para cada função $F: \operatorname{dom}(F) \rightarrow \mathbb{Z} \backslash\{0\} \operatorname{com} \operatorname{dom}(F) \in[\kappa]^{<\omega}$, se tenha $\sum_{\xi \in \operatorname{dom}(F)} F(\xi) x_{\xi} \neq 0$. Observamos que para isso basta com que, para algum $\beta<\mathfrak{c}$, seja

$$
\sum_{\xi \in \operatorname{dom}(F)} F(\xi) x_{\xi}(\beta) \neq 0 \in \mathbb{T}
$$

Se $\left\{g_{n}: n \in \omega\right\}$ é uma seqüência em $G$, para cada $n \in \omega$, temos uma funçào $\mathcal{F}(n)$ de domínio um subconjunto finito de $\kappa$,

$$
\mathcal{F}(n): \operatorname{dom} \mathcal{F}(n) \rightarrow \mathbb{Z} \backslash\{0\}
$$

tal que $g_{n}=\sum_{\xi \in d o m \mathcal{F}(n)} \mathcal{F}(n)(\xi) x_{\xi}=x_{\mathcal{F}(n)}$. Portanto a seqüência $\left\{g_{n}: n \in \omega\right\}$ é representada pela função

$$
\mathcal{F}: \omega \rightarrow \bigcup_{E \in[\kappa]<\omega \backslash\{\emptyset\}}(\mathbb{Z} \backslash\{0\})^{E}
$$


definida por $\mathcal{F}=\{(n, \mathcal{F}(n)): n \in \omega\}$.

Lema 4.2. Seja $\left\{g_{n}: n \in \omega\right\}$ uma seqüência em $G=\langle X\rangle$ que não contém uma subseqüência constante. Então existe uma seqüencia injetiva.

$$
\mathcal{F}: \omega \rightarrow \bigcup_{E \in[r]<\omega}(\mathbb{Z} \backslash\{0\})^{E}
$$

tal que $\left\{x_{\mathcal{F}(n)}: n \in \omega\right\} \subseteq\left\{g_{n}: n \in \omega\right\}$ e satisfaz uma das seguintes condiçôes:

1) $\operatorname{dom} \mathcal{F}(n) \neq \operatorname{dom} \mathcal{F}(m), \forall n, m \in \omega \operatorname{com} m \neq n$;

2) existe $E \in[\kappa]^{<\omega}$ tal que dom $\mathcal{F}(n)=E, \forall n \in \omega$, existe $\mu \in E$ tal que $\mathcal{F}(n)(\mu) \neq$ $\mathcal{F}(m)(\mu), \forall n, m \in \omega$ com $m \neq n$.

Demonstração: Se $\left\{g_{n}: n \in \omega\right\}$ não contém uma subseqüência constante, ela contém uma subseqüência injetiva. Seja $\left\{a_{n}: n \in \omega\right\} \subseteq\left\{g_{n}: n \in \omega\right\}$ injetiva, e seja

$$
\mathcal{G}: \omega \rightarrow \bigcup_{E \in[\kappa]^{<\omega} \backslash\{\emptyset\}}(\mathbb{Z} \backslash\{0\})^{E}
$$

tal que $a_{n}=x_{\mathcal{G}(n)}$.

Se existe $A \subseteq \omega$ infinito tal que $\operatorname{dom} \mathcal{G}(n) \neq \operatorname{dom} \mathcal{G}(m), \forall n, m \in \omega \operatorname{com} m \neq n$, entào enumeramos $A=\left\{n_{k}: k \in \omega\right\}$ e definimos

$$
\mathcal{F}: \omega \rightarrow \bigcup_{E \in[\kappa]<\omega \backslash\{0\}}(\mathbb{Z} \backslash\{0\})^{E}
$$

por $\mathcal{F}(k)=\mathcal{G}\left(n_{k}\right)$. Esta função $\mathcal{F}$ satisfaz 1$)$.

Se não existe um $A$ como acima então existe $B \in \omega$ infinito e $E \in[\kappa]<\omega$ tais que $\operatorname{dom} \mathcal{G}(n)=\operatorname{dom} \mathcal{G}(m)=E, \forall n, m \in \omega$.

Como $E$ é finito e a seqüência $\left\{a_{n}: n \in \omega\right\}$ é injetiva, exista $\mu \in E$ e $C \subseteq B$ infinito tal que $\mathcal{G}(n)(\mu) \neq \mathcal{G}(n)(\mu), \forall n, m \in \omega \operatorname{com} m \neq n$. 
Seja $C=\left\{n_{k}: k \in \omega\right\}$. Definimos

$$
\mathcal{F}: \omega \rightarrow \bigcup_{E \in[r]<\omega \backslash\{\emptyset\}}(\mathbb{Z} \backslash\{0\})^{E}
$$

por $\mathcal{F}(k)=\mathcal{G}\left(n_{k}\right)$. Esta função $\mathcal{F}$ satisfaz 2).

Seja $\mathcal{F}_{\alpha}: \alpha<\kappa$ uma enumeração das seq6uências injetoras

$$
\mathcal{F}: \omega \rightarrow \bigcup_{E \in[r]<\omega \backslash\{0\}}(\mathbb{Z} \backslash\{0\})^{E}
$$

que satisfazem a condiçào 1) ou a condição 2) do lema acima.

Do Lema 4.2 segue que para que o grupo $G$ seja enumeravelmente compacto é suficiente com que cada $\alpha<\kappa$ a seqüência $\left\{x_{\mathcal{F}_{a}(n)}: n \in \omega\right\}$ possua ponto de acumulação em $G$.

Fixamos $\mathcal{B}=\left\{W_{l}: l \in \omega\right\}$ uma base de abertos (arcos) para $\mathbb{T}$ tal que $\mathbb{T} \in \mathcal{B}$.

Definição 4.3. Seja $D \subseteq \kappa$ infinito e sejam as funçòes $\psi_{0}: D \rightarrow \mathcal{B}$ e $\psi_{1}: D \rightarrow \mathcal{B}$ tais que $\left\{\xi \in D: \psi_{i}(\xi) \neq \mathbb{T}\right\}$ é finito, para $i=0,1$. Dizemos que $\psi_{1} \leq \psi_{2}$ se $\overline{\psi_{1}(\xi)} \subseteq \psi_{0}(\xi)$, para todo $\xi \in D$.

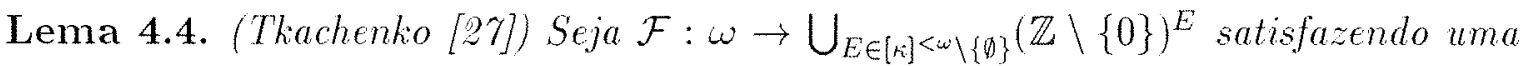
das seguintes condiçoes:

1) $\operatorname{dom} \mathcal{F}(n) \neq \operatorname{dom} \mathcal{F}(m), \forall n, m \in \omega \operatorname{com} m \neq n$;

2) existe $E \in[k]^{<\omega}$ tal que dom $\mathcal{F}(n)=E, \forall n \in \omega$, e existe $\mu \in E$ tal que $\mathcal{F}(n)(\mu) \neq$ $\mathcal{F}(m)(\mu), \forall n, m \in \omega$ com $m \neq n$.

Seja $\psi: D \rightarrow \mathcal{B}$ uma função tal que $\{\xi \in D: \psi(\xi) \neq \mathbb{T}\}$ é finito. Se $W \in \mathcal{B}$, então existe $m \in \omega$ e uma função $\psi^{\prime}: D \rightarrow \mathcal{B} \operatorname{com}\left\{\xi \in D: \psi^{\prime}(\xi) \neq \mathbb{T}\right\}$ finito tal que

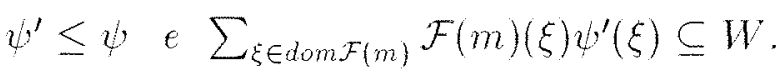




\section{Demonstração:}

caso 1: a função $\mathcal{F}$ satisfaz a condição 1).

Então existe $m_{0} \in \omega$ e $\gamma \in \operatorname{dom} \mathcal{F}\left(m_{0}\right)$ tal que $\psi(\gamma)=\mathbb{T}$. Vamos definir $\psi^{\prime}: D \rightarrow \mathcal{B}$ tal que

$$
\left\{\xi \in D: \psi^{\prime}(\xi) \neq \mathbb{T}\right\} \subseteq\{\xi \in D: \psi(\xi) \neq \mathbb{T}\} \cup \operatorname{dom} \mathcal{F}\left(m_{0}\right)=C
$$

Para cada $\xi \in D$ tal que $\psi(\xi) \neq \mathbb{T}$, fixamos $a_{\xi} \in \psi(\xi)$ e para cada

$$
\xi \in \operatorname{dom} \mathcal{F}\left(m_{0}\right) \backslash(\{\xi \in D: \psi(\xi) \neq \mathbb{T}\} \cup\{\gamma\})
$$

definimos $a_{\xi}=0$.

Vamos escolher $a_{\gamma} \in \mathbb{T}$ tal que

$$
\sum_{\xi \in d u m \mathcal{F}\left(m_{0}\right)} \mathcal{F}\left(m_{0}\right)(\xi) a_{\xi} \in W
$$

Agora, para cada $\xi \in C$, fixamos $W_{\xi} \in \mathcal{B}$ tal que $\sum_{\xi \in \text { dom } \mathcal{F}\left(m_{0}\right)} \mathcal{F}\left(m_{0}\right)(\xi) W_{\xi} \subseteq W$. $a_{\xi} \in W_{\xi}$, e $\overline{W_{\xi}} \subseteq \psi(\xi)$ para cada $\xi$ tal que $\psi(\xi) \neq \mathbb{T}$.

Definimos $\psi^{\prime}: D \rightarrow \mathcal{B}$ por $\psi^{\prime}(\xi)=\mathbb{T}$ se $\xi \in D \backslash C$ e $\psi^{\prime}(\xi)=W_{\xi}$ para todo $\xi \in C$.

caso 2: a função $\mathcal{F}$ satisfaz a condição 2).

Se $E \nsubseteq\{\xi \in D: \psi(\xi) \neq \mathbb{T}\}=C$ procedemos como no caso 1 ).

Se $E \subseteq C$, como o conjunto $\{F(n)(\mu): n \in \omega\}$ é não limitado em $\mathbb{Z}$, existe $m_{0} \in \omega$ tal que $\mathcal{F}\left(m_{0}\right)(\mu) \cdot \psi(\mu)=\mathbb{T}$.

Fixamos $a_{\xi} \in \psi(\xi), \forall \xi \in E \backslash\{\mu\}$ e escolhemos $a_{\mu} \in \psi(\mu)$ tal que

$$
\sum_{\xi \in E \backslash\{\mu\}} \mathcal{F}\left(m_{0}\right)(\xi) a_{\xi}+\mathcal{F}\left(m_{0}\right)(\mu) a_{\mu} \in W
$$

Agora procedemos como no caso 1. 
Definição 4.5. Seja $\mathbb{P}$ a família de todos os elementos

$$
p=\left(\alpha_{p},\left\{x_{p, \xi}: \xi \in D_{p}\right\},\left\{A_{p, \zeta}: \zeta \in E_{p}\right\}\right)
$$

que satisfazem:

i) $\alpha_{p} \in \omega_{1}$

ii) $D_{p} \in[\kappa]^{\omega}$;

iii) $x_{p, \xi} \in \mathbb{T}^{\alpha_{p}}$

iv) $E_{p} \in[\kappa]^{\omega}$;

v) $A_{p, \zeta} \subseteq D_{p} \cap \mathfrak{c}$

vi) $\operatorname{dom} \mathcal{F}_{\zeta}(n) \subseteq D_{p}$ para cada $n \in \omega, \zeta \in E_{p}$;

vii) $x_{p, \xi}$ é ponto de acumulação de $\left\{x_{p, \mathcal{F}_{\zeta}(n)}: n \in \omega\right\}$ para cada $\xi \in A_{p, \zeta}$ e cada $\zeta \in E_{p} ;$

viii) $\left\{x_{p, \xi}: \xi \in D_{p}\right\}$ é um conjunto livre.

Dados $p, q \in \mathbb{P}, p$ estende $q$, isto é $p \leq q$, se

a) $\alpha_{p} \geq \alpha_{q}$

b) $D_{p} \supseteq D_{q}$

c) $x_{p, \xi}\left\lceil\alpha_{q}=x_{q, \xi}\right.$ para todo $\xi \in D_{q}$;

d) $E_{p} \supseteq E_{q}$;

є) $A_{p, \zeta} \supseteq A_{q, \zeta}$ para todo $\zeta \in E_{q}$.

Lema 4.6. ( $\mathbb{P}, \leq)$ é uma ordem parcial.

Primeiro vamos verificar que $\mathbb{P} \neq \emptyset$.

Seja $p=\left(\alpha_{p},\left\{x_{p, \xi}: \xi \in D_{p}\right\},\left\{A_{p, \zeta}: \zeta \in E_{p}\right\}\right)$, com

$\alpha_{p}=\omega$

$D_{p} \in\left[\kappa \backslash \omega_{1}\right]^{\omega}, D_{p}=\left\{\xi_{n}: n \in \omega\right\}$

$x_{p, \xi_{n}} \in \mathbb{T}^{\omega}, \forall n \in \omega$, é tal que $x_{p, \xi_{n}}(n) \neq 0 \in \mathbb{T}$ e $x_{p, \xi_{n}}(m)=0$ se $n \neq m$; 
$E_{p} \in[\kappa]^{\omega}$ tal que $\operatorname{dom} \mathcal{F}_{\zeta}(n) \subseteq D_{p}, \forall n \in \omega, \forall \zeta \in E_{p} ;$

$A_{p, \zeta}=\emptyset, \forall \zeta \in E_{p}$.

$p$ assim definido é um elemento de $\mathbb{P}$.

É claro que a relação $\leq$ é reflexiva e antisimétrica. Vamos verificar que é também transitiva. Sejam $p, q, r \in \mathbb{P}$ com $p \leq q$ e $q \leq r$. Entào $x_{p, \xi} \Gamma_{\alpha_{r}}=x_{q, \xi} \Gamma_{\alpha_{r}}=x_{r, \xi}$.

Lema 4.7. A ordem parcial $\mathbb{P}$ é $\omega_{1}$ fechada.

Demonstração: Seja $\left\{p_{n}: n \in \omega\right\} \subseteq \mathbb{P}$ tal que $p_{n+1} \leq p_{n}, \forall n \in \omega$. Definimos

$$
p_{\omega}=\left(\alpha_{p_{\omega}},\left\{x_{p_{\omega}, \xi}: \xi \in D_{p_{\omega}}\right\},\left\{A_{p_{\omega}, \zeta}: \zeta \in E_{p_{\omega}}\right\}\right),
$$

onde $\alpha_{\omega}=\sup \left\{\alpha_{p_{n}}: n \in \omega\right\}, D_{p_{\omega}}=\bigcup_{n \in \omega} D_{p_{n}}, x_{p_{\omega}, \xi}=\bigcup_{\xi \in D_{p_{n}}} x_{p_{n}, \xi}, E_{p_{\omega}}=\bigcup_{n \in \omega} E_{p_{n}}$, $A_{p_{w}, \zeta}=\bigcup_{n \in \omega} A_{p_{n}, \zeta}$.

Afirmamos que $p_{\omega} \in \mathbb{P}$. Temos que verificar as condições (vii) e (viii).

Sejam $\zeta \in E_{p_{\omega}}$ e $\xi \in A_{p_{\omega}, \zeta}$. Seja $V$ uma vizinhança aberta (básica) de $x_{p_{\omega}, \xi}$ em $\mathbb{T}^{\alpha_{p_{\omega}}}$. Como $V$ depende de uma quantidade finita $\beta_{1}<\ldots<\beta_{k}<\alpha_{p_{\omega}}$ de coordenadas e pela definiçào de $\alpha_{p_{\omega}}$, existe $n_{0} \in \omega$ tal que para todo $n \geq n_{0}$ é $\alpha_{p_{n}}>\beta_{k}, \xi \in D_{p_{n}}$ e $\zeta \in E_{p_{n}} . \operatorname{Logo} x_{p_{n}, \xi} \in \pi_{\alpha_{p_{n}}}(V)$ e $\pi_{\alpha_{p_{n}}}(V) \cap\left\{x_{p_{n}, \mathcal{F}_{\zeta}(k)}: k \in \omega\right\} \neq \emptyset$. Portanto $V \cap\left\{x_{p_{\omega}, \mathcal{F}_{\zeta}(k)}: k \in \omega\right\} \neq \emptyset$, e temos a condiçäo (vii).

Seja $E \in\left[D_{p_{\omega}}\right]^{<\omega}$ e seja $f: E \rightarrow \mathbb{Z} \backslash\{0\}$. Queremos $\sum_{\xi \in E} f(\xi) x_{p_{\omega}, \xi} \neq 0$.

Pela definição de $D_{p_{\omega}}$, temos que existe $n \in \omega$ tal que $E \subseteq D_{p_{n}}$ e portanto $\sum_{\xi \in E} f(\xi) x_{p_{n}, \xi} \neq 0$

Lema 4.8. Seja $r=\left(\alpha_{r},\left\{x_{r, \xi}: \xi \in D_{r}\right\},\left\{A_{r, \zeta}: \zeta \in E_{r}\right\}\right)$ satisfazendo as condiçóes (i) a (viii) da definição da ordem $\mathbb{P}$. Então existe $p \in \mathbb{P}$ tal que $p$ "estende" $r$, isto é as condições a) - e) são satisfeitas.

Demonstração: Vamos definir $p \in \mathbb{P}$ tal que $\alpha_{p}=\alpha_{r}+\omega, D_{p}=D_{r}, E_{p}=E_{r}$, $A_{p, \zeta}=A_{r, \zeta}$ para todo $\zeta \in E_{p}$ and $\left.x_{p, \xi}\right|_{\alpha_{r}}=x_{r, \xi}$ para todo $\xi \in D_{p}$. 


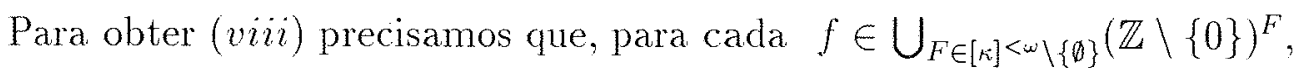

$$
\sum_{\xi \in \operatorname{dom}(f)} f(\xi) x_{p, \xi} \neq 0
$$

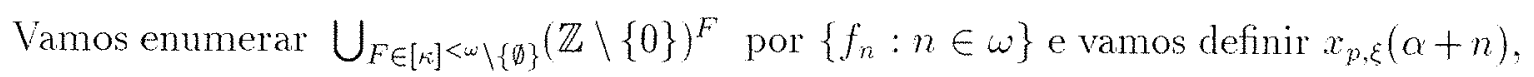
por indução, para cada $n \in \omega$ e para cada $\xi \in D_{p}$, tal que a condição (viii) seja satisfeita e $\sum_{\xi \in \operatorname{dom}\left(f_{n}\right)} f_{n}(\xi) x_{p, \xi}(\alpha+n)=x_{p, f_{n}}(\alpha+n) \neq 0$.

Suponhamos que a condiçào (vii) é satisfeita por $\left\{x_{p, \xi} \uparrow_{\alpha_{r}+n}: \xi \in D_{p}\right\}$. Para cada $\zeta \in E_{p}$ e cada $\mu \in A_{p, \zeta}$, seja $B_{\zeta, \mu}$ um subconjunto infinito de $\omega$ tal que $\left\{x_{p, \mathcal{F}_{\zeta}(m)} \uparrow_{a_{r}+n}: m \in B_{\zeta, \mu}\right\}$ converge para $x_{p, \mu} \uparrow_{\alpha_{r}+n}$.

Seja $\left\{B_{\zeta, \mu, k}: k \in \omega\right\}$ uma partição de $B_{\zeta, \mu}$ tal que $\left|B_{\zeta, \mu, k}\right|=\omega$ para todo $k \in \omega$.

Lembramos que $\mathcal{B}=\left\{W_{l}: l \in \omega\right\}$ é uma base de abertos para $\mathbb{T}$. Enumeramos todos os possiveis pares $\left(B_{\zeta, \mu, k}, W_{l}\right)$ onde $\zeta \in E_{p}, \mu \in A_{p, \zeta}$ e $k, l \in \omega$ por $\left\{\left(B_{\zeta_{t}, \mu_{t}, k_{t}}, W_{l_{t}}\right): k \in \omega\right\}$.

Vamos definir por indução uma família decrescente de funções $\psi_{t}: D_{p} \rightarrow \mathcal{B}$ tais que $\left\{\xi \in D_{p}: \psi_{t}(\xi) \neq \mathbb{T}\right\}$ é finito.

Seja $\psi_{0}: D_{p} \rightarrow \mathcal{B}$ tal que dom $\left(f_{n}\right) \subseteq\left\{\xi \in D_{p}: \psi_{0}(\xi) \neq \mathbb{T}\right\}$ e $0 \notin \sum_{\xi \in \operatorname{dom}\left(f_{n}\right)} f_{n}(\xi) \psi_{0}(\xi)$ Do Lema 4.4 aplicado a $\mathcal{F}_{\zeta}\left\lceil_{\zeta_{\zeta_{0}, \mu_{0}, k_{0}}}\right.$, segue que existe $m_{0} \leq B_{\zeta_{0}, \mu_{0}, h_{0}}$ e $\psi_{1} \leq \psi_{0} \mathrm{com}$ $\left\{\xi \in D_{p}: \psi_{1}(\xi) \neq \mathbb{T}\right\}$ finito tais que

$$
\sum_{\xi \in \operatorname{dom} \mathcal{F}_{\zeta_{0}}\left(m_{0}\right)} \mathcal{F}_{\zeta}\left(m_{0}\right)(\xi) \psi_{1}(\xi) \subseteq W_{l_{0}} .
$$

Por indução, usando o Lema 4.4, definimos $\left\{\psi_{t}: t \in \omega\right\}$ e $\left\{m_{t}: t \in \omega\right\}$ tais que $\psi_{t+1} \leq \psi_{t} \mathrm{e}$

$$
\sum_{\xi \in \operatorname{dom}_{\mathcal{F}_{\zeta_{t}}}\left(m_{t}\right)} \mathcal{F}_{\zeta}\left(m_{t}\right)(\xi) \psi_{t}(\xi) \subseteq W_{l_{t}} .
$$

Para cada $\xi \in D_{p}$, definimos $x_{p, \xi}\left(\alpha_{r}+n\right) \in \bigcap_{t \in \omega} \overline{\psi_{t}(\xi)}$. Então $\left\{x_{p, \xi}\left\lceil_{\alpha_{r}+n+1}: \xi \in D_{p}\right\}\right.$ satisfaz (vii) e $\sum_{\xi \in d o m\left(f_{n}\right)} f_{n}(\xi) x_{p, \xi}\left(\alpha_{r}+n\right) \neq 0$. 
Lema 4.9. (CH) $\mathbb{P}$ é $\omega_{2}$-c.c.

Demonstração: Seja $\left\{p_{\beta}: \beta<\omega_{2}\right\}$ um subconjunto de $\mathbb{P}$. Usando o Lema do $\Delta-$ sistema, como vale CH, temos que existe $I \in\left[\omega_{2}\right]^{\omega_{2}}$ e $D \in[\kappa] \leq \omega$ tais que $D_{p_{\beta}} \cap D_{p_{\gamma}}=D$ para todo par $\{\beta, \gamma\} \in[I]^{2}$. Como $|I|=\omega_{2}$, podemos assumir que existe $\alpha<\omega_{1}$ tal que $\alpha_{p_{\beta}}=\alpha$ para cada $\beta \in I$. Também podemos assumir que $\left|\left\{x_{p_{\beta}, \xi}: \beta \in I\right\}\right|=1$ para cada $\xi \in D$.

Fixamos $\beta, \gamma \in I$. Seja $D_{r}=D_{p_{\beta}} \cup D_{p_{\gamma}}$. Definimos $x_{r, \xi}$ como $x_{p_{\beta}, \xi}$ se $\xi \in D_{p_{\beta}}$ ou $x_{p_{\gamma}, \xi}$ se $\xi \in D_{p_{\gamma}} \backslash D$.

Seja $E_{r}=E_{p_{\beta}} \cup E_{p_{\gamma}}$, definimos $A_{r, \zeta}$ como $A_{p_{\beta}, \zeta}$ se $\zeta \in E_{p_{\beta}} \backslash E_{p_{\gamma}} ; A_{p_{\gamma}, \zeta}$ se $\zeta \in E_{p_{\gamma}} \backslash E_{p_{\beta}}$ ou $A_{p_{\beta}, \zeta} \cup A_{p_{\gamma}, \zeta}$ se $\zeta \in E_{p_{\beta}} \cap E_{p_{\gamma}}$.

Vemos que $r=\left(\alpha,\left\{x_{r, \xi}: \xi \in D_{r}\right\},\left\{A_{r, \zeta}: \zeta \in E_{r}\right\}\right)$ satisfaz as condições (i) a (vii) da definição da ordem $\mathbb{P}$. Então pelo Lema 4.8, $\exists p \in \mathbb{P}$ tal que $\alpha_{p} \geq \alpha, D_{p} \supseteq D_{r}$, $x_{p, \xi} \uparrow_{\alpha_{r}}=x_{r, \xi} \forall \xi \in D_{r}, E_{p} \supseteq E_{r}$ e $A_{p, \zeta} \supseteq A_{p, \zeta}, \forall \zeta \in E_{r}$. Portanto $p$ estende $p_{\beta}$ e $p_{\gamma}$.

Lema 4.10. O conjunto $\mathcal{D}_{\alpha, \zeta, \zeta, \mu}=\left\{p \in \mathbb{P}: \alpha_{p} \geq \alpha, \xi \in D_{p}, \zeta \in E_{p} \wedge A_{p, \zeta} \backslash \mu \neq \emptyset\right\}$ é denso f $m \mathbb{P}$, para cada $\alpha, \mu<\omega_{1} \in \xi$. $\zeta<\kappa$.

Demonstração: Seja $q$ um elemento arbitrário de $\mathbb{P}$.

Se $\zeta \in E_{q}$, definimos $E_{r}=E_{q}$. Seja $\theta \in(\mu, \mathfrak{c})$ tal que $\theta \notin D_{q} \cup\{\xi\}$, e seja $D_{r}=D_{q} \cup\{\theta\} \cup\{\xi\}$. Definimos $x_{q, \theta}$ como um ponto de acumulaçào da seqüência $\left\{x_{q, \mathcal{F}_{\zeta}(n)}: n \in \omega\right\}$. Se $\xi \in D_{r} \backslash D_{q}$ define $x_{q, \xi}=0 \in \mathbb{T}^{\alpha_{q}}$.

Se $\zeta \notin E_{q}$, escolhemos $\theta \in(\mu, c) \backslash\left(D_{q} \cup \bigcup_{n \in \omega^{\prime}} \operatorname{dom} \mathcal{F}_{\zeta}(n) \cup\{\xi\}\right)$ e pomos

$$
D_{r}=D_{\eta} \cup\left(\bigcup_{n \in \omega} \operatorname{dom} \mathcal{F}_{\zeta}(n)\right) \cup\{\theta, \xi\}
$$


Se $\psi \in D_{r} \backslash\left(D_{q} \cup\{\theta\}\right)$, definimos $x_{q, \psi}=0 \in \mathbb{T}^{\alpha_{q}}$. Definimos $x_{q, \theta}$ como um ponto de acumulação da seqüência $\left\{x_{q, \mathcal{F}_{\zeta}(n)}: n \in \omega\right\}$.

Em ambos casos, definimos $A_{r, p}=A_{q, p}$ para cada $\rho \in E_{r} \backslash\{\zeta\}$ e $A_{r, \zeta}=A_{q, \zeta} \cup\{\theta\}$. Se $\alpha_{q} \geq \alpha$, seja $x_{r, \eta}=x_{q, \eta}$ para cada $\eta \in D_{1}$.

Se $\alpha_{q}<\alpha$, seja $x_{r, \eta}=x_{q, \eta} \cup\left\{(\beta, 0): \alpha_{q} \leq \beta<\alpha\right\}$ para cada $\eta \in D_{r}$. Seja $\alpha_{r}=\max \left\{\alpha, \alpha_{q}\right\}$.

Então $r=\left(\alpha_{r},\left\{x_{r, \eta}: \eta \in D_{r}\right\},\left\{A_{r, p}: \rho \in E_{r}\right\}\right)$ satisfaz as condições (i) a (vii) da definiçào da ordem $\mathbb{P}$. Portanto pelo Lema 4.8 , existe $p \in \mathbb{P}$, "abaixo" de $r$. Logo $p \leq q$ e $p \in \mathcal{D}_{\alpha, \xi, \zeta, \mu}$.

\section{Prova do Exemplo 4.1:}

Seja $\mathrm{M}$ um modelo enumerável de ZFC + GCH tal que $\kappa \in M, \mathbb{P} \in M$ e $\kappa \geq \omega_{2}$ en M.

Seja $\mathbb{G}$ um conjunto genérico para a ordem parcial $\mathbb{P}$.

Lema 4.11. (CH) O forcing com $\mathbb{P}$ preserva cardinais.

Demonstração: Sabemos que a ordem parcial $\mathbb{P}$ é $\omega_{1}$-fechada e portanto pelo Corolário 1.28 , o forcing preserva $\omega_{1}$. Como $\mathbb{P}$ é $\omega_{2}-$ c.c. e $\omega_{2}$ é regular em $\mathrm{M}$, o forcing preserva cardinais maiores ou iguais a $\omega_{2}$.

Lema 4.12. $\mathbb{P}$ nâo introduz conjuntos enumeráveis novos.

Demonstração: Temos que mostrar que se $A \in \mathrm{M}, B \in \mathrm{M}[\mathbb{G}], B \subseteq A$ e $B$ é enumerável em $\mathrm{M}[\mathbb{G}]$, entào $B \in \mathrm{M}$. 
Seja, então, $B \subseteq A$ e $B$ é enumerável em $M[\mathbb{G}]$. Temos que existe uma função $f: \omega \rightarrow A$ tal que $f(\omega)=B$ e $f \in M[\mathbb{G}]$.

Como $\mathbb{P}$ é $\omega_{1}$-fechado, pelo Lema 1.27 temos que $f \in M$. Então $\omega \in M$ e $f \in M$ implicam que $B=f(\omega) \in \mathrm{M}$.

Para cada $\xi, \zeta \in \kappa$, seja $x_{\xi}=\bigcup_{p \in G \wedge \xi \in D_{p}} x_{p, \xi}$ e $A_{\zeta}=\bigcup_{p \in \mathbb{G} \wedge \zeta \in E_{p}} A_{p, \zeta}$. Pelo Lema 4.10 , cada $x_{\xi}$ é uma função em $\mathbb{T}^{\omega_{1}}$ e o conjunto $\left\{x_{\beta}: \beta<\kappa\right\}$ é livre. Também, cada $A_{\zeta}$ tem cardinalidade $\mathfrak{c}$, e para cada $\mu \in A_{\zeta} . x_{\mu}$ é ponto de acumulaçào da seqüência $\left\{x_{\mathcal{F}_{\zeta}(n)}: n \in \omega\right\}$.

Fixamos $\lambda \in[c, k]$. O grupo gerado por $\left\{x_{\beta}: \beta<\lambda\right\}$ é abeliano livre. Vamos verificar que $\left\langle\left\{x_{\beta}: \beta<\lambda\right\}\right\rangle$ é enumeravelmente compacto.

Seja $\left\{g_{n}: n \in \omega\right\}$ uma seqüência em $\left\langle\left\{x_{\beta}: \beta<\lambda\right\}\right\rangle$. Como $\mathbb{P}$ nào adiciona novos conjuntos enumeráveis, temos que $\left\{g_{n}: n \in \omega\right\} \in$ M. Pelo Lema 4.2, ou $\left\{g_{n}: n \in \omega\right\}$ contém uma subseqüência constante ou existe $\zeta<\kappa$ tal que

$$
\left\{x_{\mathcal{F}_{\zeta}(n)}: n \in \omega\right\} \subseteq\left\{g_{n}: n \in \omega\right\}
$$

No primeiro caso, é claro que $\left\{g_{n}: n \in \omega\right\}$ possui ponto de acumulação. No segundo caso, usando o Lema 4.10, podemos achar $p \in \mathbb{G}$ tal que $\zeta \in E_{p}$ e $A_{p, \zeta} \neq \emptyset$. Portanto para cada $\mu \in A_{p, \zeta}, x_{p, \mu}$ é ponto de acumulação de $\left\{x_{p, \mathcal{F}_{\zeta}(n)} n \in \omega\right\}$. E como $p \in \mathbb{G}$ temos que $x_{\mu}$ é ponto de acumulação de $\left\{x_{\mathcal{F}_{\zeta}(n)}: n \in \omega\right\}, \forall \mu \in A_{\zeta} \subseteq \mathfrak{c} \leq \lambda$.

Lema 4.13. (CH) $\mathfrak{c}=\omega_{1} \quad \epsilon \quad 2^{\omega_{1}}=2^{\mathfrak{c}}=\kappa \epsilon m \mathrm{M}[\mathbb{G}]$.

Demonstração: $\mathbb{P}$ não introduz novos conjuntos enumeráveis, portanto o valor de $\mathfrak{c}=2^{\omega}$ é o mesmo em $M$ e em $M[\mathbb{G}] . \operatorname{Em} M, \mathfrak{c}=\omega_{1}$, e como $\omega_{1}$ é preservado temos que $\mathfrak{c}=\omega_{1}$ em $M[\mathbb{G}]$. 
Sabemos que $\kappa=\left|\left\{x_{\beta}: \beta<\kappa\right\}\right| \leq\left|\mathbb{T}^{\omega_{1}}\right|=\mathfrak{c}^{\omega_{1}}=\omega_{1}^{\omega_{1}} \leq\left(2^{\omega_{1}}\right)^{\omega_{1}}=2^{\omega_{1}}$ em M[G].

Falta provar que $2^{\omega_{1}} \leq \kappa$ em $M[\mathbb{G}]$.

Como $\kappa$ é regular e $\kappa \geq \omega_{2}$ em $M$ e $M$ é um modelo de $\mathrm{ZFC}+\mathrm{GCH}$, temos que $\kappa^{\omega_{1}}=\kappa \mathrm{e} \kappa^{\omega}=\kappa \mathrm{em} \mathrm{M}$.

Como $\mathbb{P}$ é $\omega_{2}-$ c.c., toda anticadéia em $(\mathbb{P}, \leq)$, que é elemento de $M$, tem cardinalidade menor ou igual a $\omega_{1}$. Então, em $M$, o número total de anticadéias em $(\mathbb{P}, \leq)$ é menor ou igual a $\left|\mathbb{P}^{\omega_{1}}\right|=\kappa^{\omega_{1}}=\kappa$. Logo o número de "nice-names"em M para subconjuntos de $\omega_{1}$ é menor ou igual a $\kappa$. Pela demonstração do Lema 1.30 temos que $2^{\omega_{1}} \leq \kappa \mathrm{em}$ $\mathrm{M}[\mathbb{G}]$. ([25], Cap VII, Lema 5.3.) 


\section{Apêndice A}

\section{Ultrafiltros seletivos de $\omega$}

Neste apêndice, vamos apresentar a demonstraçào da Proposição 1.33. Este teorema. na versão mais geral, para ultrafiltros sobre ordinal infinito $\alpha$ qualquer pode ser lida em [4]. Aqui apresentamos a versão específica para ultrafiltros sobre $\omega$.

Todos os ultrafiltros considerados neste anexo sào pontos de $\beta \omega \backslash \omega=\omega^{*}$, isto é. sào ultrafiltros livies de $\omega$.

Definição A.1. Dizemos que dois ultrafiltros $u, v$ são equivalentes, e escrevemos $u \equiv v$, se existe uma bijeção $f: \omega \rightarrow \omega$ tal que $u=\bar{f}(v)$, onde $\bar{f}: \beta \omega \rightarrow \beta \omega$ denota a. extensão de Stone-Čech de $f$.

Lembramos que $u \leq v$ se existe uma funçào $f: \omega \rightarrow \omega$ tal que $\bar{f}(v)=u$, i.e. se $u=\left\{X: f^{-1}[X] \in v\right\}$. É a chamada ordem de Rudin-Keisler em $\omega^{*}$.

Vamos verificar que se $u \leq v$ e $v \leq u$, então $u \equiv v$.

Proposição A.2. Se $f: \omega \rightarrow \omega \in u=\bar{f}(u)$, então $\{n: f(n)=n\} \in u$. 
Demonstração: Consideramos os conjuntos $X=\{n: f(n)<n\}$ e $Y=\{n: f(n)>$ $n\}$ e vemos que nenhum deles é um elemento de $u$. Para cada $n \in Y$ seja $l(n)$ o comprimento da seqüência máxima $n<f(n)<f(n)^{2}<\ldots$ Sejam $Y_{0}=\{n \in Y$ : $l(n)$ épar $\}, \quad Y_{1}=\{n \in Y: l(n)$ é impar $\}$ e $Z=\left\{n \in Y: n<f(n)<f(n)^{2}<\right.$ $\ldots$, é infinita $\}$.

Temos que $Y_{0} \notin u$ pois $Y_{0} \cap f^{-1}\left[Y_{0}\right]=\emptyset$. De igual forma $Y_{1} \notin u$.

Se $x, y \in Z$ definimos $x \sim y$ se existem naturais $k$ e $m$ que satisfazem $f^{k}(x)=f^{m}(y)$. Para cada $x \in Z$, seja $a_{x}$ um representante de $\{y: y \sim x\}$, e seja

$$
l(x)=\min \left\{k+m: f^{k}(x)=f^{m}\left(a_{x}\right)\right\}
$$

Definimos $Z_{0}=\{x \in Z: l(x)$ é par $\} \quad$ e $Z_{1}=\{x \in Z: l(x)$ é impar $\}$. Então $f^{-1}\left[Z_{1}\right] \cap Z=Z_{0}$ e portanto nem $Z_{0}$ e nem $Z_{1}$ são elementos de $u$. De forma análoga se verifica que $X \notin u$.

Proposição A.3. Se u ev são dois ultrafiltros que satisfazem $u \leq v \in v \leq u$, entăo $u \equiv v$.

Demonstração: Como $u \leq v$, existe uma função $g: \omega \rightarrow \omega$ tal que $\bar{g}(v)=u$. Analogamente como $v \leq u$, existe uma função $h: \omega \rightarrow \omega$ tal que $\bar{h}(u)=v$. Segue que $\overline{g \circ h}(u)=u$, portanto $A=\{n: g \circ h(n)=n\} \in u$ pela proposiçào A.2. Em particular se $f=g \circ h\left\lceil\right.$ a função $f \uparrow_{A}$ é injetora. Seja $\left\{A_{0}, A_{1}\right\}$ uma partição de $A$ onde $\left|A_{0}\right|=\left|A_{1}\right|=\omega$. Assumimos que $A_{0} \in u$. Sabemos que $\omega=\left|\omega \backslash A_{0}\right|=\left|\omega \backslash f\left[A_{0}\right]\right|$. Seja $\pi$ uma permutação de $\omega$ tal que $\pi\left[\omega \backslash A_{0}\right]=\omega \backslash f\left[A_{0}\right]$ e $\pi \uparrow_{A_{0}}=f \uparrow_{A_{0}}$. Então $\bar{\pi}(u)=\bar{f}(u)=u$.

Proposição A.4. Para u um ultrafiltro livre de w são equivalentes:

(a) u é minimal na ordem de Rudin-lieisler; 
(b) u é seletivo;

(c) para cada partição $\left\{P_{k}: k \in \omega\right\}$ de $\omega$, ou existe $k \in \omega$ tal que $P_{k} \in p$ ou existe $A \in u$ tal que $\left|A \cap P_{k}\right| \leq 1 \forall k \in \omega$;

(d) toda coloraçầo $F:[\omega]^{2} \rightarrow\{0,1\}$ possui um conjunto homogêneo $A \in u$.

\section{Demonstração:}

(a) $\Rightarrow(\mathrm{b})$

Seja $f: \omega \rightarrow \omega$ uma funçào.

Se $\bar{f}(u)$ é um ultrafiltro principal, então existe $a \in \omega$ tal que $\{a\} \in \bar{f}(u)$, isto é $f^{-1}[\{a\}] \in u$. Neste caso $A=f^{-1}[\{a\}]$.

Se $\bar{f}(u)$ é um ultrafiltro livre por definiçào $\bar{f}(u) \leq u$ e por hipótese $u$ é minimal, logo $\bar{f}(u) \sim u$. Portanto existe uma permutaçào $\pi$ de $\omega$ que satisfaz $\overline{\pi \circ f}(u)=u$. Seja $A=\{n: \pi \circ f(n)=n\} \in u$. A funçào $f \Gamma_{A}$ é injetora.

(b) $\Rightarrow(c)$

Seja $\left\{P_{k}: k \in \omega\right\}$ uma partição de $\omega$. Definimos a função $f: \omega \rightarrow \omega$ por $f(x)=k \Leftrightarrow$ $x \in P_{k}$. Por hipótese, existe $A \in u$ satisfazendo uma das condiçòes de (b).

(c) $\Rightarrow(d)$

Afirmação: Se $X_{0} \supseteq X_{1} \supseteq X_{2} \supseteq \ldots$ são elementos de $u$, então existe uma sequência $a_{0}<a_{1}<a_{2}<\ldots$ tal que $\left\{a_{n}: n \in \omega\right\} \in u$ e $a_{n+1} \in X_{a_{n}} \forall n \in \omega$.

Demonstração: Sejam $X_{0} \supseteq X_{1} \supseteq X_{2} \supseteq \ldots$ elementos de $u$. Como u é P-ponto, existe $Y \in u$ tal que $Y \backslash X_{n}$ é finito, para todo $n \in \omega$. Definimos a sequência crescente $\left\{y_{n}: n \in \omega\right\}$ em $Y$ como segue:

$y_{0}=\min \left\{k \in Y:\{y \in Y: y>k\} \subseteq X_{0}\right\}$

$y_{n+1}=\min \left\{k \in Y: k>y_{n},\{y \in Y: y>k\} \subseteq X_{y_{n}}\right\}$.

Para cada $n$, seja $A_{n}=\left\{y \in Y: y_{n}<y \leq y_{n+1}\right\}$. Por (c) existe $\left\{z_{n}: n \in \omega\right\} \in p$, com $z_{n} \in A_{n}$ para cada $n \in \omega$. 
Como $z_{n+2}>y_{n+2}$, temos que $z_{n+2} \in X_{y_{n+1}}$, e como $y_{n+1} \geq \tilde{z}_{n}$ temos que $X_{y_{n+1}} \subseteq X_{z_{n}}$, portanto $z_{n+2} \in X_{z_{n}}$

Sejam, para cada $n \in \omega, a_{n}=z_{2 n}$ e $b_{n}=z_{2 n+1}$. Então $\left\{a_{n}: n \in \omega\right\} \in u$ ou $\left\{b_{n}: n \in \omega\right\} \in u$. Aquele que for elemento de $p$ satisfaz a afirmaçào.

Seja uma coloração $F: \omega^{2} \rightarrow\{0,1\}$. Para cada $a \in \omega$, seja $F_{a}: \omega \backslash\{a\} \rightarrow\{0,1\}$ a função definida por $F_{a}(x)=F(x, a)$. Então para cada $a \in \omega$, existe $H_{a} \in p$ tal que $F_{a}\left\lceil H_{a}\right.$ é constante. Logo existem $r \in\{0,1\}$ e e $A \in u$ tais que $F(x, a)=r \forall x \in H_{a}$, $\forall a \in A$.

Para cada $n \in \omega$ seja $x_{n}=A \cap H_{0} \cap \ldots \cap H_{n}$. Pela afirmação podemos considerar uma seqüencia crescente $H=\left\{a_{n}: n \in \omega\right\} \in u$, com $a_{n+1} \in H_{a_{n}}, \forall n \in \omega$.

Para cada $n \in \omega a_{n} \in A$ e $\left\{a_{m}: m>n\right\} \subseteq H_{a_{n}}$, portanto $F\left(x, a_{n}\right)=r \forall x \in\left\{a_{m}\right.$ : $m>n\}$. Segue que $F$ é constante em $H^{2}$.

$(\mathrm{d}) \Rightarrow(\mathrm{c})$

Seja u um ultrafiltro satisfazendo $(d)$, e seja $\left\{P_{k}: k \in \omega\right\}$ uma partição de $\omega$. Suponhamos que $P_{k} \notin u$, para todo $k \in \omega$, definimos a coloração $F: \omega^{2} \rightarrow\{0,1\}$ por $F(x, y)=0$ se $\{x, y\} \subseteq P_{k}$ para algum $k \in \omega$ e $F(x, y)=1$ caso contrário.

Por hipótese podemos considerar $A \in u$ tal que a funçào $F$ é constante em $A^{2}$. Como estamos supondo $P_{k} \notin u$, para todo $k \in \omega$, temos que $\left|A \cap P_{k}\right| \leq 1$ para cada $k \in \omega$. (c) $\Rightarrow$ (a)

Seja u um ultrafiltro satisfazendo (c), vamos provar que para toda função $f: \omega \rightarrow \omega$ tal que $\bar{f}(u) \in \omega^{*} \operatorname{temos} \bar{f}(u) \sim u$.

Seja então $f$ uma função como acima. Para cada $k \in \omega$ seja $P_{k}=f^{-1}[\{k\}]$. Então $\left\{P_{k}: k \in \omega\right\}$ é uma partiçào de $\omega$, e como $P_{k} \notin u \forall k \in \omega$, por (c) temos que existe $A \in u$ tal que $\left|A \cap P_{k}\right| \leq 1, \forall k \in \omega$. Segue que $\left.f\right|_{A}$ é injetora, então usando a demonstração do Proposição A.3 temos $\bar{f}(u) \sim u$. 


\section{Apêndice B}

\section{Grupos abelianos de ordem limitada e subgrupos puros}

Duas propriedade fortemente usadas neste trabalho são os teoremas 1.4 e 1.5 . O primeiro diz que todo grupo abeliano de ordem finita é soma direta de cíclicos e o segundo diz que um subgrupo puro de ordem limitada é somando direto do grupo. Neste apêndice queremos dar uma idéia de porque isso acontece. A referência principal é o livro de Kaplansky [23].

Lembramos que um subgrupo $H$ de um grupo abeliano $G$ é puro se para cada $h \in H$ se $h$ é divisivel por $n$ em $G$, então $h$ é divisivel por $n$ em $H$.

Lema B.1. Sejam $G$ um grupo abeliano $e H$ um subgrupo puro de $G \in \pi: G \rightarrow G / H$ a projeçâo natural. Se $y \in G / H$, então existe $x \in G$ tal que ord $(x)=\operatorname{ord}(y) \epsilon$ $\pi(x)=y$.

Demonstração: Se ord $(y)=\infty$, podemos escolher qualquer $x \in \pi^{-1}(\{y\})$. Se $\operatorname{ord}(y)=n$, seja $z \in G$ tal que $\pi(z)=y$. Então $n . z \in H$. Como $H$ é puro, existe $h \in H$ tal que $n . z=n . h$. Seja $x=z-h$. Segue que $\pi(x)=y$ e ord $(x)=n$. 
Teorema B.2. Sejam $G$ um grupo abeliano, e $H$ um subgrupo puro de $G$ tal que $G / H$ é soma direta de grupos cíclicos. Então $H$ é somando direto de $G$.

Demonstração: Sabemos que $G / H=\oplus_{i \in I}\left\langle y_{i}\right\rangle$. Pelo lema B.1 existe uma família $\left\{x_{i}: i \in I\right\}$ tal que $\operatorname{ord}\left(x_{i}\right)=\operatorname{ord}\left(y_{i}\right)$ e $\pi\left(x_{i}\right)=y_{i}, \forall i \in I$.

Seja $K=\left\langle\left\{x_{i}: i \in I\right\}\right\rangle$. Afirmamos que $G=H \oplus K$ :

1) Se $t \in G$, temos que $\pi(t)=t *=\sum n_{i} \cdot y_{i}$. Portanto $t-\sum n_{i} \cdot x_{i} \in H$ e $t \in H+K$.

2) Seja $w \in H \cap K$. Por pertencer a $K, w=\sum n_{i} \cdot x_{i}$ e por pertencer a $H$, temos que $\sum n_{i} \cdot y_{i}=0$, portanto $n_{i} y_{i}=0 \forall i \in I$. Logo $w=0$.

Lema B.3. Sejam G um grupo abeliano e S um subgrupo puro de G. Se T é um subgrupo de $G$ tal que $S \subseteq T$ e T/S é puro em $G / S$, entĩo $T$ é puro em $G$.

Demonstração: Seja $t \in T$ tal que $t=n . x$, onde $x \in G$. Seja $\pi: G \rightarrow G / S \circ$ homomorfismo natural. Se $\pi(t)=t *$ e $\pi(x)=x *$, entào $t *=n . x *$ e como $T / S$ é puro em $G / S$ temos que $t *=n . y$ para algum $y \in T / S$.

Seja $t_{1} \in T$ tal que $\pi\left(t_{1}\right)=y\left(t_{1}\right.$ existe pois $\pi\lceil T: T \rightarrow T / S)$. Segue que $t=n . t_{1}+s$ para algum $s \in S$. Então $s=t-n . t_{1}=n . t-n \cdot t_{1}$ e como $S$ é puro $s=n . s_{1}$ para algum $s_{1} \in S$. Logo $t=n\left(t_{1}+s_{1}\right)$.

Lema B.4. Seja $G$ um grupo abeliano primário satisfazendo $r^{k} G=0$. Seja $x \in G$ tal que ord $(x)=r^{k}$. Entâo $\langle x\rangle$ é puro em $G$.

Demonstração: Observamos que por ser $G u m r$-grupo, $\langle x\rangle$ é divisivel por todo inteiro primo com $r$, portanto só precisamos nos preocupar com as potências de $r$. Suponhamos que $r^{i} . x=r^{j} . y$, onde $i<k$ e $y \in G$.Se $j \leq i$ não temos nada a fazer. Se $j>i$, temos que $r^{k} . y=r^{k-j}\left(r^{j} \cdot y\right)=r^{k-j}\left(r^{i} \cdot x\right)$ e $k-j+i<k$ 
Lema B.5. Sejam $G$ um grupo abeliano $e S$ um subgrupo de $G$. Se $\pi: G \rightarrow G / S$ é o homomorfismo natural e $x \in G$ é tal que ord $(x)=\operatorname{ord}(\pi(x))$, entäo a soma $\langle x\rangle+S$ é soma direta.

Demonstração: Suponhamos que $n . x \in S$ para algum inteiro n. Então $n . \pi(x)=0$ e portanto $\operatorname{ord}(\pi(x))\lceil n$, da hipótese segue que ord $(x) \uparrow n, \log$ o $n . x=0$.

Lema B.6. Seja $S$ um subgrupo puro de $G \operatorname{com} n S=0$. Então $(S+n G) / n G$ é puro $e m G / n G$.

Demonstração: Suponhamos que $x=m y$, onde $x \in(S+n G) / n G, y \in G / n G$, e $m$ é um inteiro. Temos que provar que $x$ é múltiplo de $m$ dentro de $(S+n G) / n G$. Seja $\pi: G \rightarrow G / n G$ o homomorfismo natural e sejam $s \in(S+n G)$ e $t \in G$ tais que $\pi(s)=x$ e $\pi(t)=y$. Observamos que podemos considerar $s \in S$.

Entào temos que $s=m t+n z$, onde $z \in G$.

Seja $d$ o máximo divisor comum de $m$ e $n$. Então $m=d m_{1}$ e $n=d n_{1}, \operatorname{com} m_{1}$ e $n_{1}$ primos entre si. Portanto existem inteiros $a$ e $b$ tais que $a \cdot m_{1}+b \cdot n_{1}=1$. Substituindo em $s=m t+n z$ temos que sé máltiplo de $d$ ein $G$ e conó subgrupo puro, $s=d . s_{1}$, $\operatorname{com} s_{1} \in S$. Logo

$$
s=d \cdot s_{1}=d\left(a \cdot m_{1}+b \cdot n_{1}\right) s_{1}=m \cdot a \cdot s_{1}+n \cdot b \cdot s_{1}=m \cdot a \cdot s_{1} .
$$

Segue que $x$ é múltiplo de $m$ em $(S+n G) / n G$.

Lema B.7. Sejam $S$ e $T$ subgrupos de $G$ com $S \cap T=0$, e suponhamos que $(S+T) / T$ é somando direto de G/T. Entâo Ś é somando direto de $G$.

Demonstração: Seja $R / T$ o somando direto de $G / T$ que complementa $(S+T) / T$. Temos que $R+(S+T)=G$ e $R \cap(S+T)=T$ Vamos ver que $G=S \oplus R$. 
Como $T \subset R$, temos que $S+R=S+T+R=G$. Também $(R \cap S) \subset R \cap(S+T)=T$. Portanto $R \cap S \subset T \cap S=0$.

Demonstração do Teorema 1.4: Como todo grupo de torção é soma direta de grupos primários podemos assumir que $G$ é um $r$-grupo.

Dado um subconjunto $A$ de $G$, dizemos que $A$ é puro se $\langle A\rangle$ é um subgrupo puro de G.

Usando o Lema de Zorn obtemos um subconjunto $\left\{x_{i}: i \in I\right\}$ puro e independente maximal. Vamos provar que $\left\langle\left\{x_{i}: i \in I\right\}\right\rangle=G$, isto é que $G=\Theta_{i \in I}\left\langle x_{i}\right\rangle$.

Suponhamos por absurdo que $\left\langle\left\{x_{i}: i \in I\right\}\right\rangle \neq G$ e seja $S=\left\langle\left\{x_{i}: i \in I\right\}\right\rangle$. Então $G / S$ é um grupo primário de ordem limitada. Seja $y \in G / S$ de ordem máxima. Pelo Lema B.1, seja $x \in G$ tal que ord $(x)=\operatorname{ord}(y)$ e $\pi(x)=y$, onde $\pi G \rightarrow G / S$ é o homomorfismo natural.

Seja $T=\langle x\rangle+S$. Pelo Lema B.4 temos que $T=\langle x\rangle \oplus S$. Observamos que $\pi(T / S)=$ $\langle y\rangle$ que é puro em $G / S$. Pelo Lema B.3 T é puro em $G$, isto é $\left\{x_{i}: i \in I\right\} \cup\{x\}$ é um subconjunto independente puro de $G$, contradizendo a maximalidade de $\left\{x_{i}: i \in I\right\}$.

Demonstração do Teorema 1.5: Seja $S$ subgrupo puro de ordem limitada do grupo $G$. Suponhamos que $n S=0$. Pelo Lema B.6 temos que $(S+n G) / n G$ é puro em $G / n G$. Como $G / n G$ é de ordem limitada, pelo Teorema 1.4 é soma direta de grupos cíclicos, e o mesmo acontece com todos os seus subgrupos. Pelo Teorema B.2 $(S+n G) / n G$ é somando direto de $G / n G$. Observamos que $S \cap n G=0$. Então aplicando o Lema. B.7 $\operatorname{com} T=n G$, temos que $S$ é somando direto de $G$. 


\section{CONSIDERAÇÕES FINAIS}

Para finalizar, gostariamos de fazer alguns comentários que tem a ver com a continuação do nosso trabalho.

No capítulo 3 , mostramos quais grupos abelianos de torção $G$, de cardinalidade $\mathfrak{c}$, admitem para cada inteiro positivo $p$ uma topologia e grupo, com $G^{p}$ enumeravelmente compacto e tal que existe $q>p \operatorname{com} G^{q}$ não enumeravelmente compacto. Achamos que é possível trocar $q>p$ por $p+1$ nesta demonstração, por estarmos trabalhando com grupos abelianos de torção limitada.

Comentamos na introdução que Dikranjan e Shakhmatov [9] classificaram, num modelo de forcing, os grupos abelianos de cardinalidade menor ou igual a $2^{\mathfrak{c}}$ que admitem uma topologia de grupo enumeravelmente compacta e sem sequências convergentes. Continuando nesta linha de trabalho, pretendemos classificar, num modelo de forcing, os grupos abelianos de cardinalidade menor ou igual a $2^{\mathfrak{c}}$ que admitem uma topologia de grupo enumeravelmente compacta e sem sequências convergentes e cujo cuadrado não é enumeravelmente compacto. 


\section{Referências Bibliográficas}

[1] Castro Pereira I., Tomita A.H., A countably compact free Abelian group whose size has countable cofinality, Aceito em Appl. Gen. Topology.

[2] Comfort W.W., Topological groups, Handbook of set-theoretic topology (K. Kunen, J.E. Vaughan eds.), North-Holland, Amsterdam, 1984, pp. 1143-1263.

[3] Comfort W.W. Problems on topological groups and other homogeneous spaces, Open Problems in Topology (J. van Mill and G.M. Reeds, eds.), North holland, 1990, pp. 311-347.

[4] Comfort W.W., Negrepontis S. The theory of Ultrafilters, Springer-Verlag, 1974.

[5] Comfort W.W., Robertson L.C. Cardinality constraints for pseudocompact and for totally dense subgroups of compact Abelian groups, Pacific J. Math.119 (1985) pp. $265-285$.

[6] Comfort W.W., Ross K.A. Pseudocompactness and uniform continuity in topological groups, Pacific J. Math. 16 (1966) pp. 483-496.

[7] Comfort W.W., van Mill J. Concerning connected, pseudocompact Abelian groups, Topology Appl. 33 (1989), pp. 21-45. 
[8] Dikranjan D., Shakhmatov D. Algebraic structure of the pseudocompact groups, Memoirs Amer. Math. Soc. 133/633, April 1998, pp. viii+83.

[9] Dikranjan D., Shakhmatov D., Forcing hereditarily separable compact-like group topologies on Abelian groups, Preprint (2003).

[10] Dikranjan D., Tkachenko M., Algebraic structure of small countably compact Abelian groups, Forum Math. 15 (2003), pp. 811-837.

[11] van Douwen E.K., The product of two countably compact topological groups, Trans. Amer. Math. Soc. 262 (1980), pp. 417-427.

[12] van Douwen E.K., The weight of pseudocompact (homogeneous) space whose cardinality has countable cofinality, Proc. Amer. Math. Soc. 80 (1980), pp. 678-682.

[13] Engelking R., General Topology, 2nd edition. Heldermann Verlag, Berlin, 1989.

[14] Fuchs L., Infinite Abelian groups, Vil.I. Academic Press, New York, 1970.

[15] Garcia-Ferreira S., Tomita A.H., Watson S., Countably compact groups from a selective ultrafilter, aceito em Proc. Amer. Math. Soc.

[16] Ginsburg J., Saks V., Some applications of ultrafilters in topology, Pacific J. Math. $\mathbf{5 7}$ (1976), pp. 199-205.

[17] Hajnal A., Juhasz I., A separable normal topological group need not be Lindeloff, Gen. Topology Appl. 6 (1976), pp. 199-205.

[18] Harrison D., Infinite Abelian groups and homological methods, Ann. Math.(2) 69 (1959), pp. 366-391. 
[1.9] Hart K., van Mill J., A countably compact group $H$ such that $H \times H$ is not countably compact. Trans. Amer. Math. Soc. 323 (1991) pp. 811-821.

[20] Hewitt E., Ross K.A., Abstract Harmonic Analysis. Springer Verlag, BerlinHeidelberg-New York 1970.

[21] Hulanicki A., Algebraic structure of compact Abelian groups, Bull. Acad. Polon. Sci. Sér. Sci. Math. Astr. Phys. 6 (1958), pp. 71-73.

[22] Jech T., Set Theory. Academic Press, New York, 1978.

[23] Kaplansky I., Infinite Abelian groups. Univ. of Mich. Press, Ann Arbor, 1954.

[24] Koszmider P., Tomita A.H., Watson S., Forcing countably compact group topologies on a larger free Abelian group, Proceedings of the 15th. Summer Conference in General Topology and its Aplications/1st Turkish International Conference on Topology and its Applications (Oxford OH/ Istambul 2000). Topology Proc 25 (2000), Summer pp. 563-574.

[25] Kunen K., Set Theory - an introduction to the independence proofs. vol. 102 of Studies in Logic and the Foundations of Mathematics. North Holland Publishing Company, Amsterdam, 1980.

[26] Robinson D.J.F., A Course in the theory of Groups, Springer-Verlag, Berlin, 1982.

[27] Tkachenko M.G., Countably compact and pseudocompact topologies on free Abelian groups, Izvestiya VUZ. Matematika, Vol 31 (1990), n.5, pp. 68-75.

[28] Tomita A.H., A group under MA coutable whose square is countably compact but whose cube is not, Topology Appl. 91 (1999), n.2, pp. 91-104. 
[29] Tomita A.H., Countable compactness and finite powers of topological groups without convergent sequences, aceito em Topology Appl.

[30] Tomita A.H., Watson S., Ultraproducts, p-limits amd antichains on the Comfort group order, aceito em Topology Appl.

[31] Tomita A.H., Two countably compact groups: one of size $\aleph_{\omega}$ and the other of weight $\aleph_{\omega}$ without non-trivial convergent sequences, Proc. Amer. Math. Soc.131 (2003), n.8, pp. 2617-2622.

[32] Tomita A.H., A solution to Comfort's question on the countable compactness of powers of a topological group, Preprint.

[33] Tomita A.H., Junqueira L. Topologia geral Notas de aula do IME/USP, 1997.

[34] Vaughan J.E., Countably compact and sequentially compact spaces, Handbook of set-theoretic topology (K. Kunen, J.E. Vaughan eds.), North-Holland, Amsterdam, 1984, pp. 1143-1263. 